\title{
Teoria do Controle Ótimo para \\ Equações Integrais Lineares do \\ tipo Volterrà-Stieltjes : Um \\ Princípio do Máximo
}

Luis Antônio Fernandes de Oliveira

Orientador : Prof. Dr. Luciano Barbanti

Tese apresentadá ao Instituto de Matemática e Estatística- USP, para a obtenção do título de Doutor em Matemática Aplicada

São Paulo, maio de 1997

Durante o programa de doutoramento o autor teve o suporte financeiro do $C N P q-C A P E S$ 
Este exemplar corresponde à redaçẫo da tese devidamentte corrigida e apresentada por Leis Antõnio Termanidesde Oliveira e aprovada pela Comissão Juilgadora.

São Paulo, 20 de outubro de 1997

Banca E*aminadona:

Pfof. Dr. Luciano Barbanti(orientadør) - IME-USP

Prof. Dr. Pedro Aladas Tonellij - IMIE-USP

Prof. Dr. Marco Antonio Raupp - LNEC

Prof. Dr. Antonioferrinardelkzé - DM-UFSCar

Pfof. Dr. Rodney Carlos Bassanei í IMIECIC-UINICAMP 
À minha esposa Roseli.

companheira de todos os caminhos, aos meus filhos Fábio e Ricardo, depositários das nossas esperanças e aos meus pais Angelo e Deolinda, condiçâa inicial de tudo 
Agradeço

ao amigo Luciano, pela orientação, incentivo e paciência a mim dispensados ao longo desta jornada,

à minha esposa Roseli, por ter compartilhado comigo as angústias e as alegrias destas pequenas descobertas,

aos meus filhos Fábio e Ricardo, pela compreensão quanto ao tempo de convívio que lhes foi roubado,

à minha sogra Da. Rosa, pelo carinho e pelo apoio .

aos colegas do Departamento de Matemática da FEIS-UNESP, pelo incentivo, aos professores do IME-USP, pela acolhida,

à CAPES e ao CNPq, pelo apoio financeiro. 


\title{
Resumo
}

Neste trabalho estudamos existência de controles ótimos para um sistema governado por uma Equação Integral Linear de Volterra-Stieltjes agindo em espaços de Banach X com estados e controles no conjunto das funções regradas de $[0, \mathrm{~T}]$ em $\mathrm{X}$, e considerando funcionais do tipo linear no seu espaço dual. A integral considerada é do tipo Dushinik e o núcleo de Volterra K pertence ao espaço de todos os operadores de semivariação uniformemente limitada em X que são fracamente regrados. Também levamos em conta uma restrição linear nas soluções, que têm uma representação integral. Uma caracterização geométrica precisa dos conjuntos de atingibilidade leva-nos a um Princípio do Máximo. Finalmente, fazemos aplicações deste resultado.

\begin{abstract}
In this work we study the existence of optimal controls for a system driven by a Volterra-Stieltjes Linear Integral equation acting on Banach spaces $\mathrm{X}$ with states and controls maps racing in the set of all regulated functions from $[0, \mathrm{~T}]$ into $\mathrm{X}$, and considering functional of linear type in its dual space. The integral considered is the Dushinik type and the Volterra's kernel belongs to the space of all uniformly bounded semivariation operators in $\mathrm{X}$ that are weakly regulated. We are also taking on consideration a linear constraint on the solution set which is represented in a integral profile. The precise characterization of the attainable sets in the process will lead us to the synthesis of a Maximum Principle. Finally, applications of the result are take on care.
\end{abstract}




\section{Indice}

Introdução. .. $\mathrm{i}$

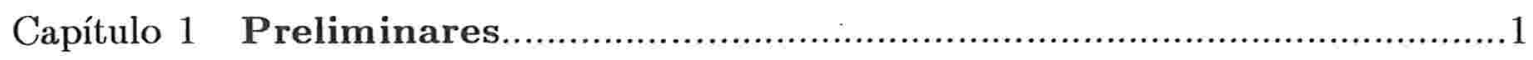

1.1 Funções Regradas e a Integral Interior......................................................

1.2 Representação de Funcionais e Operadores...............................................11

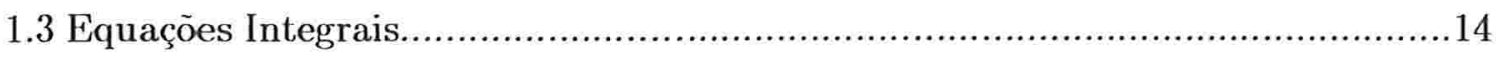

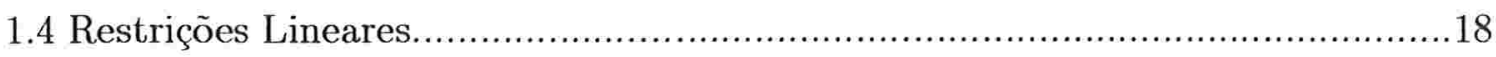

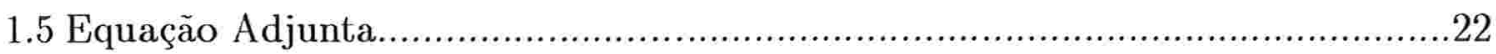

Capítulo 2 Controles Admissíveis e o Conjunto de Atingibilidade..............27

2.1 Transmissão de Propriedades Geométricas...............................................27

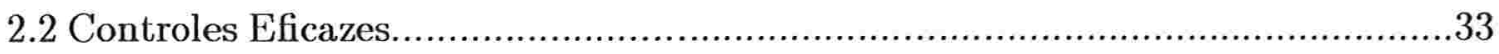

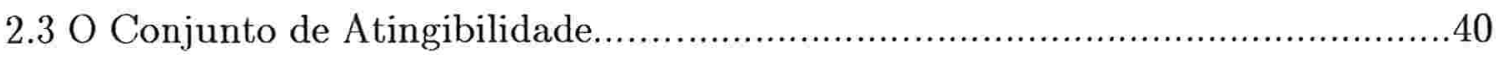

Capítulo 3 Existência de Controles Ótimos : Um Princípio do Máximo....56

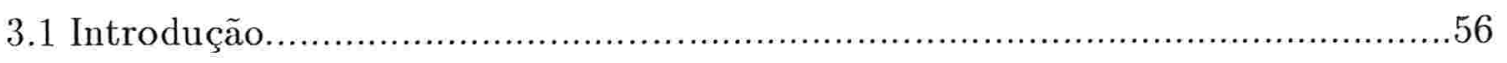

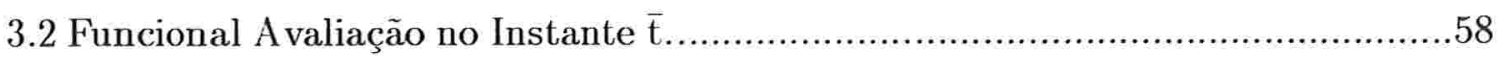

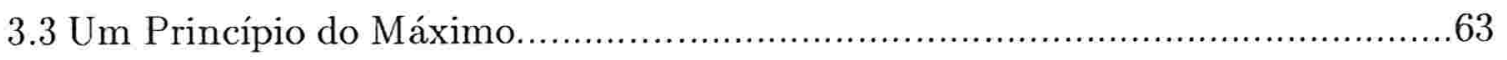

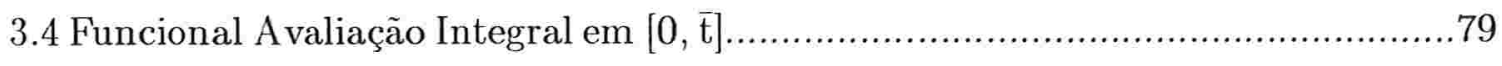

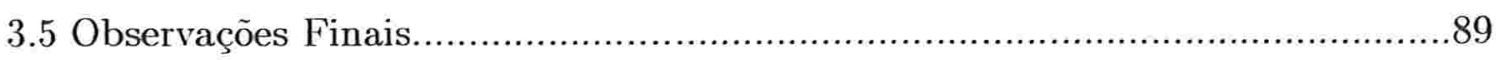

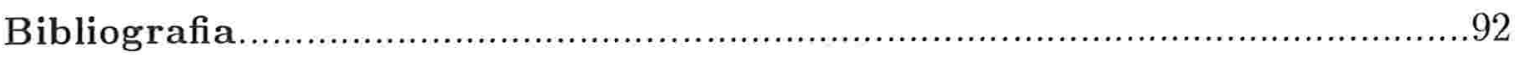




\section{Introdução}

$\mathrm{Na}$ década de 40, da necessidade de se dirigir um sistema evolutivo a um estado desejado, surge, como um tópico especial da área de equações diferenciais, a teoria do controle ótimo.

As descobertas do Princípio do Máximo e do Método de Programação Dinâmica acabaram dando novos rumos à Teoria do Controle Ótimo e ao Cálculo das Variações.

$\mathrm{Na}$ década de 60 a teoria do controle para processos determinísticos atingiu um certo grau de completude e estabilidade. Em sintonia com tal desenvolvimento, começam a aparecer alguns textos básicos cujo objetivo é o de sintetizar os resultados obtidos até então. Entre estes, destaca-se o de E.B. Lee e L. Markus, de 1967([11]).

A partir daí, multiplicaram-se tanto as aplicaçōes da teoria a áreas distintas do conhecimento, tais como engenharia, economia, biologia, etc., como as extensões dentro da própria matemática, por exemplo, a equações definidas em espaços de dimensão infinita.

Em teoria do controle, está-se interessado no comportamento de um sistema sujeito a influências externas. A cada uma destas influências, que chamaremos de controles ou entradas, o sistema produz uma resposta ou saidas, que carrega o estado inicial a um estado alvo pré-fixado. Em termos físicos, podemos ver os controles como forças aplicadas 
ao estado do sistema com o objetivo de levá-lo ao estado alvo.

O problema geral de controle ótimo pode ser descrito por quatro tipos de dados :

(1) O processo ou equação que define como o sistema evolui com o tempo;

(2) O estado inicial e o estado alvo do sistema;

(3) A classe de controles admissíveis, ou seja, em que ambiente será permitido buscar os controles;

(4) O custo ou índice de desempenho associado a cada controle.

Uma das principais questões na teoria do controle é a existência de um controle ótimo, isto é, um controle que leve o sistema até o estado alvo, de modo a otimizar um certo custo. Além disso, se tal controle existe, será que é único? Neste caso, podemos exibir um modo de construí-lo, isto é, ele pode ser sintetizado?

O nosso objetivo nesta tese consiste em estudar existência de controle ótimo, para funcionais custo que descreveremos ainda nesta introdução, para um sistema cuja dinâmica é regida por uma equação integral de VolterraStieltjes linear, da qual as soluções estão sujeitas a uma restrição linear, no ambiente dos espaços de Banach, tudo acompanhado de uma interpretação "geométrica". O resultado final será a apresentação de um Princípio do Máximo.

Em outras palavras, estudamos o sistema $(\mathrm{K})+\left(\mathrm{F}_{\alpha}\right)$, sendo

$$
\mathrm{x}(\mathrm{t})-\mathrm{x}_{\mathrm{o}}+\int_{0}^{\mathrm{t}} \cdot \mathrm{d}_{\mathrm{s}} \mathrm{K}(\mathrm{t}, \mathrm{s}) \cdot \mathrm{x}(\mathrm{s})=\mathrm{u}(\mathrm{t}), 0 \leq \mathrm{t} \leq \mathrm{T}<\infty,
$$




$$
\mathrm{F}_{\alpha}[\mathrm{x}]=\int_{0}^{\mathrm{t}} \cdot \mathrm{d} \alpha(\mathrm{s}) \cdot \mathrm{x}(\mathrm{s})=0
$$

para $\mathrm{K} \in \mathrm{G}_{0}^{\sigma} \cdot \mathrm{SV}^{\mathrm{u}}([0, \mathrm{~T}] \times[0, \mathrm{~T}], \mathrm{L}(\mathrm{X})), \alpha \in \mathrm{SV}_{0}([0, \mathrm{~T}], \mathrm{L}(\mathrm{X})), \mathrm{x}, \mathrm{u} \in \mathrm{G}([0, \mathrm{~T}], \mathrm{X})$, o espaço das funções regradas de $[0, T]$ com valores no espaço de Banach $X, e z \in X$. As integrais que aparecem em $(\mathrm{K})+\left(\mathrm{F}_{\alpha}\right)$ são integrais de Dushnik ou integral interior.

A teoria acerca da equação $(K)$, existência de solução regrada, dependência contínua, teoremas de representação de operadores,equação adjunta, etc., foi desenvolvida por Hönig nas publicações [7],[8],[5] e [10] citadas na bibliografia, e da qual fazemos uma síntese no capítulo 1 .

Os espaços de Banach $\mathrm{G}_{0}^{\sigma} \cdot \mathrm{SV}^{\mathrm{u}}([0, \mathrm{~T}] \times[0, \mathrm{~T}], \mathrm{L}(\mathrm{X}))$ e $\mathrm{SV}_{0}([0, \mathrm{~T}], \mathrm{L}(\mathrm{X}))$ são isométricos a subespaços de operadores lineares contínuos. Isto significa que a cada um de tais operadores corresponde um núcleo em $\mathrm{G}_{0}^{\sigma} \cdot \mathrm{SV}^{\mathrm{u}}$ ou em $\mathrm{SV}_{0}$, o que lhes garantirá uma representação integral.

A importância de se estudar Teoria do Controle para o sistema considerado é a abrangência da equação (K). Equações Diferenciais Ordinárias Lineares, Equações Diferenciais Parciais Lineares, Equações Diferenciais com Retardamento, Equações Impulsivas, Equações Funcionais do Tipo Neutro, via Teorema de Representação de Operadores Causais, são instâncias da equação (K). Em [8], por exemplo, Hönig mostra que a cada gerador de um $C_{0}$-semigrupo corresponde um núcleo $\alpha$, e que portanto sistemas com tal dinâmica podem ser vistos sob a ótica de $(\mathrm{K})$. No parágrafo 1.3 , exibimos um exemplo de equação diferencial parcial linear que pode ser tratada neste contexto.

Por sua vez, a restrição linear $\left(\mathrm{F}_{\alpha}\right)$ é geral e traduz, por exemplo, condições iniciais, condições de periodicidade, condições de fronteira, etc., sobre as soluções de (K).

Portanto, da generalidade do sistema $(\mathrm{K})+\left(\mathrm{F}_{\alpha}\right)$ podemos inferir o caráter unificador 
do estudo da Teoria do Controle para processos geridos por esta dinâmica. Este estudo tem sido feito por Barbanti.Em [1], por exemplo, é tratada a questão da controlabilidade e controlabilidade aproximada para as equações do tipo (K).

O capítulo 1 é dedicado a definir a integral interior, enunciar propriedades e resultados mais conhecidos (com exceção da proposição 1.1.16) da teoria sobre a equação (K), bem como do espaço das funções regradas. Além disso, incluimos alguns exemplos que julgamos pertinentes.

No capítulo 2 juntamos as propriedades geométricas dos conjuntos de controles admissíveis e do conjunto dos estados atingíveis.

Ambos são uma preparação aos resultados apresentados no capítulo 3.

No capítulo 3 apresentamos o problema de controle ótimo para os seguintes tipos de funcionais :

$$
\begin{gathered}
\mathcal{L}(\overline{\mathrm{t}}) \mathrm{u}=\left\langle\varphi, \mathrm{x}_{\mathrm{u}}(\overline{\mathrm{t}})>,\right. \\
\mathcal{L}(0, \overline{\mathrm{t}}) \mathrm{u}=\int_{0}^{\overline{\mathrm{t}}} \cdot\left\langle\mathrm{d} \gamma(\mathrm{s}), \mathrm{x}_{\mathrm{u}}(\mathrm{s})>\right.
\end{gathered}
$$

sendo $\mathrm{x}_{\mathrm{u}}$ a solução do sistema $(\mathrm{K})+\left(\mathrm{F}_{\alpha}\right)$ associada ao controle $\mathrm{u}$ tomados num subconjunto de $G([0, T], X)$.

A parte central deste trabalho, o Princípio do Máximo, será considerada no paragráfo 3.3 .

$\mathrm{Na}$ literatura não encontramos algo no mesmo contexto. Em grande parte, os trabalhos com equações integrais de Volterra se restringem à dimensão finita ou, quando tratam de espaços de Banach, impõem, por exemplo, reflexibilidade de X (v. [4]). Por isto 
entendemos que os resultados ora apresentados são uma contribuição à teoria das equações integrais.

A continuação natural deste trabalho deixa aberto de imediato os campos para o estudo dos problemas :

. Funcionais Quadráticos

. Funcionais Convexos

. Programação Dinâmica 


\section{Capítulo 1}

\section{Preliminares}

\subsection{Funções Regradas e a Integral Interior}

As definições e resultados que enunciamos a seguir podem ser encontrados nas referências [10],[5] e [7]. Faremos aqui um breve resumo da teoria sobre a equação (K) com o objetivo de facilitar a leitura deste texto. Os principais resultados de [5] são reescritos para o caso particular em que $\mathrm{Y}=\mathrm{X}$.

Sejam X e Y espaços de Banach. Vamos denotar por L(X,Y) o espaço de Banach das aplicações lineares contínuas de $X$ em $Y$. Em particular, $L(X)=L(X, X)$ e $X^{\prime}=L(X, I R)$. Como estamos interessados em processos evolutivos em tempo finito, todas as definições e resultados a seguir serão considerados no intervalo $[0, \mathrm{~T}]$, para $\mathrm{T}>0$ fixado.

Começamos definindo que tipo de funções vamos considerar neste trabalho, isto é, o universo das entradas e saidas do nosso processo de controle. 
1.1.1 Definição Dizemos que $\mathrm{f}:[0, \mathrm{~T}] \rightarrow \mathrm{X}$ é uma função regrada se, para todo $\mathrm{t} \in[0, \mathrm{~T}[(\mathrm{t} \in] 0, \mathrm{~T}])$, existe

$$
f\left(t_{+}\right)=\lim _{\tau \downarrow t} f(\tau)\left(f\left(t_{-}\right)=\lim _{\tau \uparrow t} f(\tau)\right)
$$

Em outras palavras, uma função regrada f só tem descontinuidades de primeira espécie.

Denotamos por $\mathrm{G}([0, \mathrm{~T}], \mathrm{X})$ o espaço de Banach das funções regradas $\mathrm{f}:[0, \mathrm{~T}] \rightarrow X$, munido da norma

$$
\|\mathbf{f}\|=\sup _{t \in[0, T]}\{\|\mathbf{f}(\mathrm{t})\|\}
$$

Vamos destacar rapidamente algumas propriedades do conjunto $\mathrm{G}([0, \mathrm{~T}], \mathrm{X})$.

1. $\mathrm{f} \in \mathrm{G}([0, \mathrm{~T}], \mathrm{X})$ se, e somente se, f for limite uniforme de funções em escada.

2. O conjunto de descontinuidades de uma função regrada é no máximo enumerável.

3. $\mathrm{G}([0, \mathrm{~T}], \mathrm{X})$ contém o conjunto das funções contínuas $\mathrm{C}([0, \mathrm{~T}], \mathrm{X})$ e o conjunto das funções de variação limitada $\mathrm{BV}([0, \mathrm{~T}], \mathrm{X})$.

4. Toda função regrada é limitada.

5. G([0,T],X) é o "menor" espaço de Banach que contém as funções em escada.

Denotaremos por $\mathcal{D}_{\left[0, T_{]}^{1}\right.}$ o conjunto de todas as divisões de $[0, \mathrm{~T}]$,

$$
\mathrm{d}: 0=\mathrm{t}_{0}<\mathrm{t}_{1}<\ldots<\mathrm{t}_{\mathrm{n}}=\mathrm{T}
$$


sendo que $\mathrm{n}=|\mathrm{d}|$ denota a ordem da divisão $\mathrm{d}$, e por $\mathrm{G}^{-}([0, \mathrm{~T}], \mathrm{X})$ o subespaço de $\mathrm{G}([0, \mathrm{~T}], \mathrm{X})$ das funções contínuas à esquerda, isto é,

$$
\mathrm{G}^{-}([0, \mathrm{~T}], \mathrm{X})=\left\{\mathrm{f} \in \mathrm{G}([0, \mathrm{~T}], \mathrm{X}) / \mathrm{f}^{-}=\mathrm{f}\right\}
$$

$\mathrm{f}^{-}(\mathrm{t})=\mathrm{f}\left(\mathrm{t}_{-}\right)$, para todo $\left.\left.\mathrm{t} \in\right] 0, \mathrm{~T}\right]$.

1.1.2 Definição Definimos a semivariação de $\alpha:[0, \mathrm{~T}] \rightarrow \mathrm{L}(\mathrm{X}, \mathrm{Y})$ por

$$
\mathrm{SV}[\alpha]=\sup _{\mathrm{d} \in \mathrm{D}_{[\mathbf{0}, \mathbf{T}}} \mathrm{SV}_{\mathrm{d}}[\alpha]
$$

sendo que, para cada divisão d de $[0, \mathrm{~T}]$,

$$
\operatorname{SV}_{\mathrm{d}}[\alpha]=\sup \left\{\left\|\sum_{\mathrm{i}=1}^{|\mathrm{d}|}\left[\alpha\left(\mathrm{t}_{\mathrm{i}}\right)-\alpha\left(\mathrm{t}_{\mathrm{i}-1}\right)\right] \cdot \mathrm{x}_{\mathrm{i}}\right\| / \mathrm{x}_{\mathrm{i}} \in \mathrm{X},\left\|\mathrm{x}_{\mathrm{i}}\right\| \leq 1\right\}
$$

Dizemos que $\alpha$ tem semivariação limitada se $\operatorname{SV}[\alpha]<\infty$, e denotamos o espaço de Banach das funções de semivariação limitada $\alpha:[0, \mathrm{~T}] \rightarrow \mathrm{L}(\mathrm{X}, \mathrm{Y})$ tais que $\alpha(0)=0$, por $\mathrm{SV}_{0}([0, \mathrm{~T}], \mathrm{L}(\mathrm{X}, \mathrm{Y}))$, munido da norma $\|\alpha\|=\mathrm{SV}[\alpha]$.

Observemos que $\mathrm{BV}([0, \mathrm{~T}], \mathrm{L}(\mathrm{X}, \mathrm{Y})) \subset \mathrm{SV}([0, \mathrm{~T}], \mathrm{L}(\mathrm{X}, \mathrm{Y}))$, e a igualdade é válida se, e somente se, $\operatorname{dim} Y<\infty$. Em particular,

$$
\mathrm{BV}\left([0, \mathrm{~T}], \mathrm{X}^{\prime}\right)=\mathrm{SV}\left([0, \mathrm{~T}], \mathrm{X}^{\prime}\right)
$$

1.1.3 Notação Quando $\alpha \in \mathrm{BV}\left([0, \mathrm{~T}], \mathrm{X}^{\prime}\right)$ usaremos a notação

$$
\alpha(\mathrm{t}) \cdot \mathrm{x}=<\alpha(\mathrm{t}), \mathrm{x}>
$$


1.1.4 Definição Seja $\mathrm{d} \in \mathcal{D}_{[0, T\}}$. Uma divisão $\mathrm{d}^{\prime}$ é dita mais fina que $\mathrm{d}\left(\mathrm{d}^{\prime} \geq \mathrm{d}\right)$ se todo ponto $t_{\mathrm{j}}$ de $\mathrm{d}$ for um ponto de $\mathrm{d}^{\prime}$.

1.1.5 Definição Para $\alpha:[0, \mathrm{~T}] \rightarrow \mathrm{L}(\mathrm{X}, \mathrm{Y})$ e $\mathrm{f}:[0, \mathrm{~T}] \rightarrow \mathrm{X}$ definimos a integral interior ( de Dushnik) por

$$
\int_{0}^{\mathrm{T}} \cdot \mathrm{d} \alpha(\mathrm{t}) \cdot \mathrm{f}(\mathrm{t})=\lim _{\mathrm{d} \in \mathcal{D}_{[0, \mathrm{~T}]}} \sum_{\mathrm{i}=1}^{|\mathrm{d}|}\left[\alpha\left(\mathrm{t}_{\mathrm{i}}\right)-\alpha\left(\mathrm{t}_{\mathrm{i}-1}\right)\right] \cdot \mathrm{f}\left(\xi_{\mathrm{i}}\right)
$$

sendo $\left.\xi_{\mathrm{i}} \in\right] \mathrm{t}_{\mathrm{i}-1}, \mathrm{t}_{\mathrm{i}}[$, quando existir tal limite. Aqui entendemos que

$$
\mathrm{x}=\lim _{\mathrm{d} \in \mathcal{D}_{[0, T]}} \mathrm{x}_{\mathrm{d}}
$$

$\mathrm{x} \in \mathrm{X}$, se para toda vizinhança $\mathrm{V}$ de $\mathrm{x}$, existe uma divisão $\mathrm{d}_{\mathrm{V}} \in \mathcal{D}_{[0, \mathrm{~T}]}$ tal que, para toda $d$ mais fina que $d_{V}$, temos que $x_{d} \in V$.

1.1.6 Teorema $(1.10$ de $[7]) \quad$ Se $\alpha \in \mathrm{SV}([0, \mathrm{~T}], \mathrm{L}(\mathrm{X}, \mathrm{Y}))$ e $\mathrm{f} \in \mathrm{G}([0, \mathrm{~T}], \mathrm{X})$, então existe a integral

$$
\int_{0}^{\mathrm{T}} \cdot \mathrm{d} \alpha(\mathrm{t}) \cdot \mathrm{f}(\mathrm{t})
$$

e

$$
\left\|\int_{0}^{\mathrm{T}} \cdot \mathrm{d} \alpha(\mathrm{t}) \cdot \mathrm{f}(\mathrm{t})\right\| \leq \mathrm{SV}[\alpha]\left\|\mathrm{f}^{-}\right\|
$$


1.1.7 Observação Em particular, no caso em que $Y=\mathbb{R}$ temos que, para $\alpha \in \mathrm{BV}_{0}\left([0, \mathrm{~T}], \mathrm{X}^{\prime}\right)$ e $\mathrm{f} \in \mathrm{G}([0, \mathrm{~T}], \mathrm{X})$, então existe a integral

$$
\int_{0}^{\mathrm{T}} \cdot<\mathrm{d} \alpha(\mathrm{t}), \mathrm{f}(\mathrm{t})>\in \mathbb{R}
$$

A integral interior estende a integral de Riemman-Stieltjes no sentido de permitir a integração de funções $\mathrm{f}$ em relação a núcleos $\alpha$, mesmo quando $\mathrm{f}$ e $\alpha$ têm pontos de descontinuidade em comum. Lembremos que quando isto acontece a integral de RiemmanStieltjes para $\alpha$ e f pode não existir. Os dois próximos resultados e o exemplo a seguir mostram esta extensão no caso numérico.

1.1.8 Exemplo Seja $\alpha \in \mathrm{SV}([0, \mathrm{~T}], \mathrm{L}(\mathrm{X}, \mathrm{Y}))$. Suponhamos que $\mathrm{f}(\mathrm{t})=\mathrm{z}$, para todo $\mathrm{t} \in[0, \mathrm{~T}]$, sendo $\mathrm{z} \in \mathrm{X}$ fixo. Então, para uma divisão qualquer de $[0, \mathrm{~T}]$,

$$
\mathrm{d}: 0=\mathrm{t}_{0}<\mathrm{t}_{1}<\ldots<\mathrm{t}_{\mathrm{n}}=\mathrm{T}
$$

$$
\begin{aligned}
& \sum_{\mathrm{i}=1}^{|\mathrm{d}|}\left[\alpha\left(\mathrm{t}_{\mathrm{i}}\right)-\alpha\left(\mathrm{t}_{\mathrm{i}-1}\right)\right] \cdot \mathrm{f}\left(\xi_{\mathrm{i}}\right)=\left[\alpha\left(\mathrm{t}_{1}\right)-\alpha\left(\mathrm{t}_{0}\right)\right] \cdot \mathrm{z}+\left[\alpha\left(\mathrm{t}_{2}\right)-\alpha\left(\mathrm{t}_{1}\right)\right] \cdot \mathrm{z}+\ldots+\left[\alpha\left(\mathrm{t}_{\mathrm{n}}\right)-\alpha\left(\mathrm{t}_{\mathrm{n}-1}\right)\right] \cdot \mathrm{z}= \\
& \quad=\left[\alpha\left(\mathrm{t}_{1}\right)-\alpha(\underbrace{\mathrm{t}_{0}}_{0})+\alpha\left(\mathrm{t}_{2}\right)-\alpha\left(\mathrm{t}_{1}\right)+\ldots+\alpha(\underbrace{\mathrm{t}_{\mathrm{n}}}_{\mathrm{T}})-\alpha\left(\mathrm{t}_{\mathrm{n}-1}\right)\right] \cdot \mathrm{z}=[\alpha(\mathrm{T})-\alpha(0)] \cdot \mathrm{z} .
\end{aligned}
$$

Portanto,

$$
\int_{0}^{\mathrm{T}} \cdot \mathrm{d} \alpha(\mathrm{t}) \cdot \mathrm{f}(\mathrm{t})=[\alpha(\mathrm{T})-\alpha(0)] \cdot \mathrm{z}
$$


1.1.9 Lema (I.1.1 de [10/) Se existe a integral de Riemman-Stieltjes

$$
\int_{0}^{\mathrm{T}} \mathrm{d} \alpha(\mathrm{t}) \cdot \mathrm{f}(\mathrm{t})
$$

então existe a integral interior, e

$$
\int_{0}^{\mathrm{T}} \cdot \mathrm{d} \alpha(\mathrm{t}) \cdot \mathrm{f}(\mathrm{t})=\int_{0}^{\mathrm{T}} \mathrm{d} \alpha(\mathrm{t}) \cdot \mathrm{f}(\mathrm{t})
$$

1.1.10 Teorema $(I .1 .4$ de $[10 /) \quad$ Dado $\mathrm{p} \in[0, \mathrm{~T}[$, existe a integral interior

$$
\int_{0}^{\mathrm{T}} \cdot \mathrm{d} \alpha(\mathrm{t}) \cdot \mathrm{f}(\mathrm{t})
$$

se, e somente se, existirem as integrais

$$
\int_{0}^{\mathrm{p}} \cdot \mathrm{d} \alpha(\mathrm{t}) \cdot \mathrm{f}(\mathrm{t}) \quad \text { e } \quad \int_{\mathrm{p}}^{\mathrm{T}} \cdot \mathrm{d} \alpha(\mathrm{t}) \cdot \mathrm{f}(\mathrm{t})
$$

Além disso,

$$
\int_{0}^{\mathrm{T}} \cdot \mathrm{d} \alpha(\mathrm{t}) \cdot \mathrm{f}(\mathrm{t})=\int_{0}^{\mathrm{p}} \cdot \mathrm{d} \alpha(\mathrm{t}) \cdot \mathrm{f}(\mathrm{t})+\int_{\mathrm{p}}^{\mathrm{T}} \cdot \mathrm{d} \alpha(\mathrm{t}) \cdot \mathrm{f}(\mathrm{t})
$$

1.1.11 Exemplo Consideremos, para $\mathrm{p} \in] 0, \mathrm{~T}[$,

$$
\alpha(\mathrm{t})=\chi_{[\mathbf{p}, \mathbf{T}]} \quad \text { e } \quad \mathrm{f}=\chi_{\mid \mathbf{p}, \mathbf{T}]},
$$


sendo que $\chi_{\mathrm{A}}$ denota a função característica do conjunto A. Então existem as integrais de Riemman-Stieltjes

$$
\int_{0}^{\mathrm{p}} \mathrm{d} \alpha(\mathrm{t}) \cdot \mathrm{f}(\mathrm{t})=0 \quad \text { e } \quad \int_{\mathrm{p}}^{\mathrm{T}} \mathrm{d} \alpha(\mathrm{t}) \cdot \mathrm{f}(\mathrm{t})=0
$$

Pelo lema anterior, segue que existem

$$
\int_{0}^{\mathrm{p}} \cdot \mathrm{d} \alpha(\mathrm{t}) \cdot \mathrm{f}(\mathrm{t}) \quad \text { e } \quad \int_{\mathrm{p}}^{\mathrm{T}} \cdot \mathrm{d} \alpha(\mathrm{t}) \cdot \mathrm{f}(\mathrm{t})
$$

e, pelo teorema 1.1.10, existe $\int_{0}^{\mathrm{T}} \cdot \mathrm{d} \alpha(\mathrm{t}) \cdot \mathrm{f}(\mathrm{t})$. Porém, não existe a integral $\int_{0}^{\mathrm{T}} \mathrm{d} \alpha(\mathrm{t}) \cdot \mathrm{f}(\mathrm{t})$, já que $\alpha$ e f são descontínuas em p.

1.1.12 Exemplo Consideremos $\alpha_{\mathrm{p}}, \alpha_{\mathrm{T}} \in \mathrm{SV}([0, \mathrm{~T}], \mathrm{L}(\mathrm{X}))$, para $\left.\mathrm{p} \in\right] 0, \mathrm{~T}[$, dadas por

$$
\begin{aligned}
& \alpha_{\mathrm{p}}(\mathrm{s}) \cdot \mathrm{x}=\left(\chi_{[\mathrm{p}, \mathrm{T}]} \cdot \mathrm{x}\right)(\mathrm{s}), \\
& \alpha_{\mathrm{T}}(\mathrm{s}) \cdot \mathrm{x}=\left(\chi_{\{\mathrm{T}\}} \cdot \mathrm{x}\right)(\mathrm{s}),
\end{aligned}
$$

para $s \in[0, T]$ e $x \in X$.

(a) Para cada $f \in G([0, T], X)$,

$$
\begin{aligned}
& \int_{0}^{\mathrm{T}} \cdot \mathrm{d} \alpha_{\mathrm{p}}(\mathrm{s}) \cdot \mathrm{f}(\mathrm{s})=\mathrm{f}\left(\mathrm{p}_{-}\right) \\
& \int_{0}^{\mathrm{T}} \cdot \mathrm{d} \alpha_{\mathrm{T}}(\mathrm{s}) \cdot \mathrm{f}(\mathrm{s})=\mathrm{f}\left(\mathrm{T}_{-}\right)
\end{aligned}
$$


Em particular, se $f \in G^{-}([0, T], X)$,

$$
\begin{aligned}
& \int_{0}^{\mathrm{T}} \cdot \mathrm{d} \alpha_{\mathrm{p}}(\mathrm{s}) \cdot \mathrm{f}(\mathrm{s})=\mathrm{f}(\mathrm{p}) . \\
& \int_{0}^{\mathrm{T}} \cdot \mathrm{d} \alpha_{\mathrm{T}}(\mathrm{s}) \cdot \mathrm{f}(\mathrm{s})=\mathrm{f}(\mathrm{T}) .
\end{aligned}
$$

Realmente, se $\mathrm{d}: 0=\mathrm{s}_{0}<\mathrm{s}_{1}<\ldots<\mathrm{s}_{\mathrm{k}}<\ldots<\mathrm{s}_{\mathrm{n}}=\mathrm{T}$ é uma divisão de $[0, \mathrm{~T}]$, sendo $\left.\mathrm{s}_{\mathrm{k}}=\mathrm{p} \mathrm{e} \xi_{\mathrm{i}} \in\right] s_{i-1}, s_{i}[$, para $\mathrm{i}=1,2, \ldots \mathrm{n}$, então

$$
\begin{aligned}
\sum_{\mathrm{i}=1}^{\mathrm{n}}\left[\alpha_{\mathrm{p}}\left(\mathrm{s}_{\mathrm{i}}\right)-\right. & \left.\alpha_{\mathrm{p}}\left(\mathrm{s}_{\mathrm{i}-1}\right)\right] \cdot \mathrm{f}\left(\xi_{\mathrm{i}}\right)=\left[\alpha_{\mathrm{p}}\left(\mathrm{s}_{1}\right)-\alpha_{\mathrm{p}}(0)\right] \cdot \mathrm{f}\left(\xi_{1}\right)+\ldots+\left[\alpha_{\mathrm{p}}(\mathrm{p})-\alpha_{\mathrm{p}}\left(\mathrm{s}_{\mathrm{k}-1}\right)\right] \cdot \mathrm{f}\left(\xi_{\mathrm{k}}\right)+ \\
& +\left[\alpha_{\mathrm{p}}\left(\mathrm{s}_{\mathrm{k}+1}\right)-\alpha_{\mathrm{p}}\left(\mathrm{s}_{\mathrm{k}}\right)\right] \cdot \mathrm{f}\left(\xi_{\mathrm{k}+1}\right)+\ldots+\left[\alpha_{\mathrm{p}}(\mathrm{T})-\alpha_{\mathrm{p}}\left(\mathrm{s}_{\mathrm{n}-1}\right)\right] \cdot \mathrm{f}\left(\xi_{\mathrm{n}}\right)
\end{aligned}
$$

Como $\alpha_{\mathrm{p}}\left(\mathrm{s}_{\mathrm{i}}\right)=0$, para $0 \leq \mathrm{i}<\mathrm{k}, \alpha_{\mathrm{p}}\left(\mathrm{s}_{\mathrm{i}}\right)=\mathrm{I}_{\mathrm{x}}$, para $\mathrm{k} \leq \mathrm{i} \leq \mathrm{n}$, temos que

$$
\sum_{\mathrm{i}=1}^{\mathrm{n}}\left[\alpha_{\mathrm{p}}\left(\mathrm{s}_{\mathrm{i}}\right)-\alpha_{\mathrm{p}}\left(\mathrm{s}_{\mathrm{i}-1}\right)\right] \cdot \mathrm{f}\left(\xi_{\mathrm{i}}\right)=\alpha_{\mathrm{p}}(\mathrm{p}) \cdot \mathrm{f}\left(\xi_{\mathrm{k}}\right)
$$

Logo,

$$
\lim _{\mathrm{d} \in \mathcal{D}} \sum_{\mathrm{i}=1}^{|\mathrm{d}|}\left[\alpha_{\mathrm{p}}\left(\mathrm{s}_{\mathrm{i}}\right)-\alpha_{\mathrm{p}}\left(\mathrm{s}_{\mathrm{i}-1}\right)\right] \cdot \mathrm{f}\left(\xi_{\mathrm{i}}\right)=\lim _{\xi_{\mathrm{k}}{ }_{\mathrm{p}}} \mathrm{f}\left(\xi_{\mathrm{k}}\right)=\mathrm{f}\left(\mathrm{p}_{-}\right) .
$$

No caso de $\alpha_{\mathrm{T}}$, para uma divisão qualquer de $[0, \mathrm{~T}]$, temos que

$$
\sum_{\mathrm{i}=1}^{|\mathrm{d}|}\left[\alpha_{\mathrm{p}}\left(\mathrm{s}_{\mathrm{i}}\right)-\alpha_{\mathrm{p}}\left(\mathrm{s}_{\mathrm{i}-1}\right)\right] \cdot \mathrm{f}\left(\xi_{\mathrm{i}}\right)=\mathrm{f}\left(\xi_{\mathrm{n}}\right)
$$




$$
\lim _{\mathrm{d} \in \mathcal{D}} \sum_{\mathrm{i}=1}^{|\mathrm{d}|}\left[\alpha_{\mathrm{T}}\left(\mathrm{s}_{\mathrm{i}}\right)-\alpha_{\mathrm{T}}\left(\mathrm{s}_{\mathrm{i}-1}\right)\right] \cdot \mathrm{f}\left(\xi_{\mathrm{i}}\right)=\mathrm{f}\left(\mathrm{T}_{-}\right)
$$

já que $\alpha_{\mathrm{T}}\left(\mathrm{s}_{\mathrm{i}}\right) \cdot \mathrm{f}\left(\xi_{\mathrm{i}}\right)=0, \mathrm{i}=1,2, \ldots, \mathrm{n}-1$ e $\alpha_{\mathrm{T}}\left(\mathrm{s}_{\mathrm{n}}\right)=\mathrm{I}_{\mathrm{x}}$.

Dada $\alpha:[0, \mathrm{~T}] \rightarrow \mathrm{L}(\mathrm{X})$ e $\varphi \in X^{\prime}$, consideremos $\varphi \circ \alpha:[0, \mathrm{~T}] \rightarrow \mathrm{X}^{\prime}$ definida, para $\mathrm{x} \in \mathrm{X}$ e $\mathrm{s} \in[0, \mathrm{~T}]$, por

$$
[\varphi \circ \alpha](\mathrm{s}) \cdot \mathrm{x}=\varphi[\alpha(\mathrm{s}) \cdot \mathrm{x}] .
$$

1.1.13 Proposição Sejam $\alpha \in \mathrm{SV}([0, \mathrm{~T}], \mathrm{L}(\mathrm{X}))$ e $\varphi \in \mathrm{X}^{\prime}$. Então

$$
\varphi \circ \alpha \in \mathrm{BV}\left([0, \mathrm{~T}], \mathrm{X}^{\prime}\right) .
$$

Prova : Para cada $\mathrm{d} \in \mathcal{D}_{[0, \mathrm{~T}]}, \mathrm{d}: 0=\mathrm{s}_{0}<\mathrm{s}_{1}<\ldots<\mathrm{s}_{\mathrm{n}}=\mathrm{T}$,

$$
\begin{gathered}
\left\|\sum_{\mathrm{i}=1}^{|\mathrm{d}|}\left[(\varphi \circ \alpha)\left(\mathrm{s}_{\mathrm{i}}\right)-(\varphi \circ \alpha)\left(\mathrm{s}_{\mathrm{i}-1}\right)\right] \cdot \mathrm{x}_{\mathrm{i}}\right\|=\| \sum_{\mathrm{i}=1}^{|\mathrm{d}|}\left(\varphi \circ \alpha\left(\mathrm{s}_{\mathrm{i}}\right)\right) \cdot \mathrm{x}_{\mathrm{i}}-\left(\varphi \circ \alpha\left(\mathrm{s}_{\mathrm{i}-1}\right) \cdot \mathrm{x}_{\mathrm{i}} \|=\right. \\
=\| \sum_{\mathrm{i}=1}^{|\mathrm{d}|}\left[\varphi\left(\alpha\left(\mathrm{s}_{\mathrm{i}}\right) \cdot \mathrm{xi}\right)-\varphi\left(\alpha\left(\mathrm{s}_{\mathrm{i}-1}\right) \cdot \mathrm{x}_{\mathrm{i}}\right)\|=\| \sum_{\mathrm{i}=1}^{|\mathrm{d}|} \varphi\left(\left[\alpha\left(\mathrm{s}_{\mathrm{i}}\right)-\alpha\left(\mathrm{s}_{\mathrm{i}-1}\right)\right] \cdot \mathrm{x}_{\mathrm{i}}\right) \| \leq\right. \\
\leq\|\varphi\|\left\|\sum_{\mathrm{i}=1}^{|\mathrm{d}|}\left[\alpha\left(\mathrm{s}_{\mathrm{i}}\right)-\alpha\left(\mathrm{s}_{\mathrm{i}-1}\right)\right] \cdot \mathrm{x}_{\mathrm{i}}\right\| .
\end{gathered}
$$

Logo, 


$$
\begin{aligned}
& \mathrm{SV}_{\mathrm{d}}[\varphi \circ \alpha]=\sup \left\{\left\|\sum_{\mathrm{i}=1}^{|\mathrm{d}|}\left[(\varphi \circ \alpha)\left(\mathrm{s}_{\mathrm{i}}\right)-(\varphi \circ \alpha)\left(\mathrm{s}_{\mathrm{i}-1}\right)\right] \cdot \mathrm{x}_{\mathrm{i}}\right\| / \mathrm{x}_{\mathrm{i}} \in \mathrm{X},\left\|\mathrm{x}_{\mathrm{i}}\right\| \leq 1\right\} \leq \\
& \leq\|\varphi\| \sup \left\{\left\|\sum_{\mathrm{i}=1}^{|\mathrm{d}|}\left[\alpha\left(\mathrm{s}_{\mathrm{i}}\right)-\alpha\left(\mathrm{s}_{\mathrm{i}-1}\right)\right] \cdot \mathrm{x}_{\mathrm{i}}\right\| / \mathrm{x}_{\mathrm{i}} \in \mathrm{X},\left\|\mathrm{x}_{\mathrm{i}}\right\| \leq 1\right\}=\|\varphi\| \operatorname{SV}_{\mathrm{d}}[\alpha],
\end{aligned}
$$

e portanto, $\varphi \circ \alpha \in \mathrm{BV}\left([0, \mathrm{~T}], \mathrm{X}^{\prime}\right)$.

1.1.14 Observação Segue do Teorema 1.1.6 que, para toda u $\in \mathrm{G}([0, \mathrm{~T}], \mathrm{X})$, existe

$$
\int_{0}^{\mathrm{T}} \cdot\langle\mathrm{d}(\varphi \circ \alpha)(\mathrm{t}), \mathrm{u}(\mathrm{t})\rangle=\left\langle\varphi, \int_{0}^{\mathrm{T}} \cdot \mathrm{d} \alpha(\mathrm{t}) \cdot \mathrm{u}(\mathrm{t})\right\rangle
$$

De fato, para cada divisão $\mathrm{d} \in \mathcal{D}_{\{0, \mathrm{~T}\}}, \mathrm{d}: 0=\mathrm{s}_{0}<\mathrm{s}_{1}<\cdot<\mathrm{s}_{\mathrm{n}}=\mathrm{T}$, segue da linearidade de $\varphi$ que,

$$
\sum_{\mathrm{i}=1}^{|\mathrm{d}|}<(\varphi \circ \alpha)\left(\mathrm{s}_{\mathrm{i}}\right)-(\varphi \circ \alpha)\left(\mathrm{s}_{\mathrm{i}-1}\right) \cdot \mathrm{u}\left(\xi_{\mathrm{i}}\right)>=\left\langle\varphi, \sum_{\mathrm{i}=1}^{|\mathrm{d}|}\left[\alpha\left(\mathrm{s}_{\mathrm{i}}\right)-\alpha\left(\mathrm{s}_{\mathrm{i}-1}\right)\right] \cdot \mathrm{u}\left(\xi_{\mathrm{i}}\right)>\right.
$$

Como $\varphi$ é contínua,

$$
\lim _{\mathrm{d} \in \mathcal{D}} \sum_{\mathrm{i}=1}^{|\mathrm{d}|}\left\langle(\varphi \circ \alpha)\left(\mathrm{s}_{\mathrm{i}}\right)-(\varphi \circ \alpha)\left(\mathrm{s}_{\mathrm{i}-1}\right) \cdot \mathrm{u}\left(\xi_{\mathrm{i}}\right)\right\rangle=\left\langle\varphi, \lim _{\mathrm{d} \in \mathcal{D}} \sum_{\mathrm{i}=1}^{|\mathrm{d}|}\left[\alpha\left(\mathrm{s}_{\mathrm{i}}\right)-\alpha\left(\mathrm{s}_{\mathrm{i}-1}\right)\right] \cdot \mathrm{u}\left(\xi_{\mathrm{i}}\right)\right\rangle
$$




\subsection{Representação de Funcionais e Operadores}

A introdução das noções de semivariação e de integral interior ficam justificadas pelo próximo teorema de representação ( generalização do Teorema de Representação de Riez em $\left.\mathrm{C}([\mathrm{a}, \mathrm{b}])^{\prime}\right)$, que será usado para descrever, na forma integral, as restrições lineares acopladas ao nosso problema de controle ótimo.

Além dele, enunciamos também o teorema de representação para operadores causais, que garante a abrangência da equação (K).

Seja $\mathrm{f} \in \mathrm{G}([0, \mathrm{~T}], \mathrm{X})$. Definimos, para $\alpha \in \mathrm{SV}_{0}([0, \mathrm{~T}], \mathrm{L}(\mathrm{X}, \mathrm{Y}))$,

$$
\mathrm{F}_{\alpha}(\mathrm{f})=\int_{0}^{\mathrm{T}} \cdot \mathrm{d} \alpha(\mathrm{t}) \cdot \mathrm{f}(\mathrm{t})
$$

Desse modo, a cada $\alpha \in \mathrm{SV}([0, \mathrm{~T}], \mathrm{L}(\mathrm{X}, \mathrm{Y}))$ corresponde uma aplicação linear

$$
\mathrm{F}_{\alpha}: \mathrm{G}([0, \mathrm{~T}], \mathrm{X}) \rightarrow \mathrm{Y}
$$

1.2.1 Teorema (1.11 de /7/) A aplicação

$$
\alpha \in \mathrm{SV}_{0}([0, \mathrm{~T}], \mathrm{L}(\mathrm{X}, \mathrm{Y})) \rightarrow \mathrm{F}_{\alpha} \in \mathrm{L}\left(\mathrm{G}^{-}([0, \mathrm{~T}], \mathrm{X}), \mathrm{Y}\right)
$$

é uma isometria (isto é, $\left\|\mathrm{F}_{\alpha}\right\|=\mathrm{SV}[\alpha]$ ) do primeiro espaço de Banach no segundo. Além disso, 


$$
\alpha(\mathrm{t}) \cdot \mathrm{x}=\mathrm{F}_{\alpha}\left(\chi_{[0, \mathrm{t}]} \cdot \mathrm{x}\right)
$$

para todos $\mathrm{x} \in \mathrm{X}$ e $0<\mathrm{t} \leq \mathrm{T}$

Observemos que para $\mathrm{Y}=\mathbb{R}$, o teorema anterior garante que os espaços de Banach $\mathrm{BV}_{0}\left([0, \mathrm{~T}], \mathrm{X}^{\prime}\right)$ e $\mathrm{G}^{-}([0, \mathrm{~T}], \mathrm{X})^{\prime}$ são isométricos. Em outras palavras, os funcionais lineares contínuos em $\mathrm{G}^{-}([0, \mathrm{~T}], \mathrm{X})$ podem ser representados na forma integral

$$
\int_{0}^{\mathrm{T}} \cdot\langle\mathrm{d} \alpha(\mathrm{t}), \mathrm{f}(\mathrm{t})>
$$

$\operatorname{para} \alpha \in \mathrm{BV}_{0}\left([0, \mathrm{~T}], \mathrm{X}^{\prime}\right)$.

1.2.2 Notações : Para $K:[0, T] \times[0, T] \rightarrow L(X, Y)$, denotamos por

$$
\mathrm{K}^{\mathrm{t}}:[0, \mathrm{~T}] \rightarrow \mathrm{L}(\mathrm{X}, \mathrm{Y}) \text { e } \mathrm{K}_{\mathrm{s}}:[0, \mathrm{~T}] \rightarrow \mathrm{L}(\mathrm{X}, \mathrm{Y})
$$

as aplicações definidas por $\mathrm{K}^{\mathrm{t}}=\mathrm{K}(\mathrm{t}, \mathrm{s})=\mathrm{K}_{\mathrm{s}}$. Dizemos que $\mathrm{K}$ satisfaz às propriedades ,

$\left(\mathrm{G}^{\sigma}\right)$ : se K é simplesmente regrado como função da primeira variável, isto é,

$$
\mathrm{K}_{\mathrm{s}} \in \mathrm{G}([0, \mathrm{~T}], \mathrm{L}(\mathrm{X}, \mathrm{Y})), \text { para todo } \mathrm{s} \in[0, \mathrm{~T}]
$$

$\left(\mathrm{SV}^{\mathrm{u}}\right)$ : se K é uniformente de semivariação limitada como função da segunda variável, o que significa que. 


$$
\mathrm{SV}^{\mathrm{u}}[\mathrm{K}]=\sup _{0 \leq \mathrm{t} \leq \mathrm{T}} \mathrm{SV}\left[\mathrm{K}^{\mathrm{t}}\right]<\propto
$$

$\left(\mathrm{G}_{\triangle}^{\sigma}\right)$ : se $\mathrm{K}$ é simplesmente regrado sobre a diagonal, ou seja, para todo $\mathrm{x} \in \mathrm{X}$, a função

$$
\mathrm{t} \in[0, \mathrm{~T}] \rightarrow \mathrm{K}(\mathrm{t}, \mathrm{t}) \cdot \mathrm{x} \in \mathrm{Y}
$$

é regrada.

$\left(G_{0}^{\sigma}\right)$ : se $K$ é simplesmente regrado sobre a diagonal e $K(t, t)=0$, para todo $t \in[0, T]$.

$\left(\mathrm{G}_{\mathrm{I}}^{\sigma}\right)$ : se $\mathrm{K}$ é simplesmente regrado sobre a diagonal e $\mathrm{K}(\mathrm{t}, \mathrm{t})=\mathrm{I}_{\mathrm{x}}$, para todo $\mathrm{t} \in[0, \mathrm{~T}]$.

1.2.3 Definição Seja $K \in G_{0}^{\sigma} \cdot S^{u}([0, T] \times[0, T], L(X, Y))$. Para toda função $\mathrm{f} \in \mathrm{G}([0, \mathrm{~T}], \mathrm{X})$, definimos

$$
(\mathrm{k} \mathrm{f})(\mathrm{t})=\int_{0}^{\mathrm{t}} \cdot \mathrm{d}_{\mathrm{s}} \mathrm{K}(\mathrm{t}, \mathrm{s}) \cdot \mathrm{f}(\mathrm{s}), \quad 0 \leq \mathrm{t} \leq \mathrm{T}
$$

1.2.4 Observação Se K satisfaz $\left(G_{\triangle}^{\sigma}\right)$, então $k f \in G([0, T], Y)$.

1.2.5 Definição Dizemos que um operador $P \in L(G([0, T], X), G([0, T], Y))$ é causal (ou de causalidade) quando, para toda $\mathrm{f} \in \mathrm{G}([0, \mathrm{~T}], \mathrm{X})$ e todo $\mathrm{c} \in[0, \mathrm{~T}]$, 


$$
\left.f\right|_{[0, c]}=\left.0 \Rightarrow(P f)\right|_{[0, c]}=0
$$

Os operadores causais aparecem na modelagem de sistemas evolutivos cuja dinâmica depende apenas do seu comportamento passado. O próximo teorema fornece uma forma de representar os operadores causais e garante uma poderosa ferramenta no estudo da Teoria do Controle para uma ampla classe de equações.

1.2.6 Teorema (2.10 de /7/) A aplicação

$$
\mathrm{K} \in \mathrm{G}_{0}^{\sigma} \cdot \mathrm{SV}^{\mathrm{u}}([0, \mathrm{~T}] \times[0, \mathrm{~T}], \mathrm{L}(\mathrm{X}, \mathrm{Y})) \rightarrow \mathrm{k} \in \mathrm{L}\left(\mathrm{G}^{-}([0, \mathrm{~T}], \mathrm{X}), \mathrm{G}([0, \mathrm{~T}], \mathrm{Y})\right)
$$

é uma isometria do primeiro espaço de Banach sobre o subespaço dos operadores de causalidade do segundo. Além disso, se $\mathrm{x} \in \mathrm{X}$, para $\mathrm{s}, \mathrm{t} \in] 0, \mathrm{~T}]$,

$$
\mathrm{K}(\mathrm{t}, \mathrm{s}) \cdot \mathrm{x}=-\mathrm{k}\left[\chi_{\underline{l s, \mathrm{t}\}}} \cdot \mathrm{x}\right](\mathrm{t})
$$

e, para $\mathrm{t} \in[0, \mathrm{~T}]$

$$
\mathrm{K}(\mathrm{t}, 0) \cdot \mathrm{x}=-\mathrm{k}\left[\chi_{[0, t]} \cdot \mathrm{x}\right](\mathrm{t})
$$

\subsection{Equações Integrais}

Para $\mathrm{K} \in \mathrm{G}^{\sigma} . \mathrm{SV}^{\mathrm{u}}([0, \mathrm{~T}] \times[0, \mathrm{~T}], \mathrm{L}(\mathrm{X}, \mathrm{Y}))$, consideremos a equaçào 


$$
\mathrm{x}(\mathrm{t})-\mathrm{x}_{0}+\int_{0}^{\mathrm{t}} \cdot \mathrm{d}_{\mathrm{s}} \mathrm{K}(\mathrm{t}, \mathrm{s}) \cdot \mathrm{x}(\mathrm{s})=\mathrm{u}(\mathrm{t}), 0 \leq \mathrm{t} \leq \mathrm{T}
$$

sendo $\mathrm{x}, \mathrm{u} \in \mathrm{G}([0, \mathrm{~T}], \mathrm{X}), \mathrm{u}(0)=0$ e $\mathrm{x}_{0} \in \mathrm{X}$. Aqui, $\mathrm{x}(\mathrm{t})$ são os estados do sistema governado por $(\mathrm{K})$ e $\mathrm{u}(\mathrm{t})$ são as forças externas que o influenciam. Como já dissemos, a equação $(\mathrm{K})$ engloba vários tipos de equações. Vamos mostrar a seguir um exemplo que ilustra tanto a necessidade de se trabalhar em espaços de Banach como o alcance da equação (K). Neste sentido, o teorema de representação de operadores causais (Teorema 1.2.6) é essencial.

1.3.1 Exemplo Em [14], Narukawa estuda o movimento de uma corda vibrante, regido pela equação

$$
\rho(\mathrm{x})\left[\frac{\partial}{\partial \mathrm{t}^{2}} \mathrm{f}(\mathrm{t}, \mathrm{x})\right]-\frac{\partial}{\partial \mathrm{x}}\left[\mathrm{c}(\mathrm{x}) \frac{\partial}{\partial \mathrm{x}} \mathrm{f}(\mathrm{t}, \mathrm{x})\right]=\mathrm{u}(\mathrm{t}, \mathrm{x})
$$

para $0 \leq \mathrm{x} \leq \mathrm{L}, \mathrm{T}>0$ e $0 \leq \mathrm{t} \leq \mathrm{T}$. Fazendo

$$
g(t)=\left(\begin{array}{c}
f(t) \\
\frac{\partial}{\partial t} f(t)
\end{array}\right)
$$

podemos levar a equação (1.19) à equação funcional

$$
\frac{\mathrm{d}}{\mathrm{d} t} \mathrm{~g}(\mathrm{t})=\mathrm{Ag}(\mathrm{t})+\mathrm{Bu}(\mathrm{t})
$$

Aqui $g:[0 . T] \rightarrow X$. para $X=G([0, L], \mathbb{R})$, é definida, para cada $x \in[0, L]$, por

$$
g(t) \cdot x=\left(\begin{array}{c}
f(t) \\
\frac{\partial}{\partial t} f(t)
\end{array}\right) \cdot x=\left(\begin{array}{c}
f(t) \cdot x \\
\frac{\partial}{\partial t} f(t) \cdot x
\end{array}\right)=\left(\begin{array}{c}
f(t, x) \\
\frac{\partial}{\partial t} f(t, x)
\end{array}\right)=g(t, x)
$$




$$
\begin{gathered}
A=\left(\begin{array}{ll}
0 & \mathrm{I} \\
\mathrm{H} & 0
\end{array}\right), \quad[\mathrm{B} \mathrm{u}(\mathrm{t})] \cdot \mathrm{x}=\left(\begin{array}{c}
0 \\
\rho^{-1}(\mathrm{x}) \mathrm{u}(\mathrm{t}, \mathrm{x})
\end{array}\right) \\
(\mathrm{H} \mathrm{f}) \cdot \mathrm{x}=\rho^{-1}(\mathrm{x}) \frac{\partial}{\partial \mathrm{x}}\left[\mathrm{c}(\mathrm{x}) \frac{\partial}{\partial \mathrm{x}} \mathrm{f}(\mathrm{t}, \mathrm{x})\right]
\end{gathered}
$$

Desse modo A gera um $\mathrm{C}_{0}$-semigrupo. Em [8], Hönig mostra que, se $\mathrm{D}_{\mathrm{G}_{\mathrm{r}}}$ denota o domínio de A com a norma do gráfico, isto é, $\|g\|_{G_{r}}=\|g\|+\|A \cdot g\|$, a solução fraca gerada por forças $u \in G\left([0, T], D_{G_{r}}\right)$ é na verdade uma solução forte de (1.19).

Alguns exemplos de equações que são instâncias de $(\mathrm{K})$ e que, como o exemplo anterior, ilustram o alcance do estudo da teoria do controle para (K) no ambiente dos espaços de Banach, podem ser encontrados na bibliografia citada no começo deste capítulo.

Em [9], por exemplo, Hönig cita as Equações Integrais de Volterra Lineares, Equações Diferenciais Ordinárias Lineares, Equações com Retardamento, etc.

1.3.2 Definição Dizemos que $\mathrm{R} \in \mathrm{G}_{\mathrm{I}}^{\sigma} \cdot \mathrm{SV}^{\mathrm{u}}([0, \mathrm{~T}] \times[0, \mathrm{~T}], \mathrm{L}(\mathrm{X}))$ é um resolvente de $K$ se $R$ satisfizer a equação

$$
\mathrm{R}(\mathrm{t}, \mathrm{s})-\mathrm{I}_{\mathrm{x}}+{ }^{\sigma} \int_{\mathrm{s}}^{\mathrm{t}} \cdot \mathrm{d}_{\tau} \mathrm{K}(\mathrm{t}, \tau) \circ \mathrm{R}(\tau, \mathrm{s})=0,0 \leq \mathrm{s} \leq \mathrm{t} \leq \mathrm{T}
$$

Observemos que a equação anterior tem o sentido fraco, isto é,

$$
\left[\sigma \int_{\mathrm{s}}^{\mathrm{t}} \cdot \mathrm{d}_{\tau} \mathrm{K}(\mathrm{t}, \bar{\tau}) \circ \mathrm{R}(\tau, \mathrm{s})\right] \cdot \mathrm{x}=\int_{\mathrm{s}}^{\mathrm{t}} \cdot \mathrm{d}_{\tau} \mathrm{K}(\mathrm{t}, \tau) \cdot[\mathrm{R}(\tau, \mathrm{s}) \cdot \mathrm{x}] .
$$


O próximo teorema fornece condições para que a equação $(\mathrm{K})$ tenha uma única solução regrada, e que portanto, defina um processo ( linear) de controle.

1.3.3 Teorema (3.4 de (7/) Seja $\mathrm{K} \in \mathrm{G}_{0}^{\sigma} \cdot \mathrm{SV}^{\mathrm{u}}$. Se existir $\mathrm{R} \in \mathrm{G}_{\mathrm{I}}^{\sigma} \cdot \mathrm{SV}^{\mathrm{u}}$ satisfazendo

$$
\begin{aligned}
& \mathrm{R}(\mathrm{t}, \mathrm{s})-\mathrm{I}_{\mathrm{x}}+{ }^{\sigma} \int_{\mathrm{s}}^{\mathrm{t}} \cdot \mathrm{d}_{\tau} \mathrm{K}(\mathrm{t}, \tau) \circ \mathrm{R}(\tau, \mathrm{s})=0, \quad 0 \leq \mathrm{s} \leq \mathrm{t} \leq \mathrm{T} \\
& \mathrm{R}(\mathrm{t}, \mathrm{s})-\mathrm{K}(\mathrm{t}, \mathrm{s})-\mathrm{I}_{\mathrm{x}}+{ }^{\sigma} \int_{\mathrm{s}}^{\mathrm{t}} \cdot \mathrm{d}_{\tau} \mathrm{R}(\mathrm{t}, \tau) \circ \mathrm{K}(\tau, \mathrm{s})=0, \quad 0 \leq \mathrm{s} \leq \mathrm{t} \leq \mathrm{T}
\end{aligned}
$$

isto é, $K$ tem um único resolvente $R$, então, para todo $x_{0} \in X$ e toda $u \in G([0, T], X)$, a equação $(K)$ tem uma única solução regrada $\mathrm{x}_{\mathrm{u}}(\mathrm{t})$, que é dada pela fórmula

$$
\mathrm{x}_{\mathrm{u}}(\mathrm{t})=\mathrm{u}(\mathrm{t})+\mathrm{R}(\mathrm{t}, 0) \cdot \mathrm{x}_{0}-\int_{0}^{\mathrm{t}} \cdot \mathrm{d}_{\tau} \mathrm{R}(\mathrm{t}, \tau) \cdot \mathrm{u}(\tau), \quad 0 \leq \mathrm{t} \leq \mathrm{T}
$$

\subsubsection{Observação $\mathrm{O}$ conjunto}

$\mathcal{H}=\left\{\mathrm{K} \in \mathrm{G}_{0}^{\sigma} \cdot \mathrm{SV}^{\mathrm{u}} / \mathrm{K}\right.$ tem um único resolvente $\left.\mathrm{R} \in \mathrm{G}_{\mathrm{I}}^{\sigma} \cdot \mathrm{SV}^{\mathrm{u}}\right\}$

é um subconjunto aberto de $\mathrm{G}_{0}^{\sigma} \cdot \mathrm{SV}^{\mathrm{u}}$, e a aplicação $\mathrm{K} \in \mathcal{H} \rightarrow \mathrm{R}-\mathrm{I}_{\mathrm{x}} \in \mathcal{H}$ é bicontínua. Logo, se $\mathrm{K} \in \mathcal{H}$, a solução $\mathrm{x}_{\mathrm{u}}$ dada pela fórmula $(\rho)$ é uma função contínua de $\mathrm{x}_{0}$, u e $\mathrm{K}$. Segue então que

$\mathrm{u} \in \mathrm{G}^{-}([0, \mathrm{~T}], \mathrm{X})$ se, e somente se, $\mathrm{x}_{\mathrm{u}} \in \mathrm{G}^{-}([0, \mathrm{~T}], \mathrm{X})$. 
Como a aplicação $\rho: \mathrm{u} \rightarrow \mathrm{x}_{\mathrm{u}}$ é contínua, temos que $\rho^{-1}\left(\mathrm{G}^{-}([0, \mathrm{~T}], \mathrm{X})\right)$ é um subconjunto fechado de $\mathrm{G}([0, \mathrm{~T}], \mathrm{X})$.

1.3.5 Exemplo Usando a fórmula $\left(R^{*}\right)$ podemos mostrar que as respostas correspondentes aos controles

$$
\begin{aligned}
& \mathrm{u}_{\mathrm{y}}(\mathrm{t})=\mathrm{y}, \text { para } \mathrm{t} \in[0, \mathrm{~T}] \text { e } \mathrm{y} \in \mathrm{X} \text { fixo, } \\
& \mathrm{u}_{\mathrm{K}}(\mathrm{t})=-\mathrm{K}(\mathrm{t}, 0) \cdot \mathrm{x}_{0}, \text { para } \mathrm{t} \in[0, \mathrm{~T}]
\end{aligned}
$$

são dadas, respectivamente, por

$$
\begin{aligned}
& \mathrm{x}_{\mathrm{y}}(\mathrm{t})=\mathrm{R}(\mathrm{t}, 0) \cdot\left(\mathrm{y}+\mathrm{x}_{0}\right), \text { para } \mathrm{t} \in[0, \mathrm{~T}] \\
& \mathrm{x}_{\mathrm{K}}(\mathrm{t})=\mathrm{x}_{0}, \text { para } \mathrm{t} \in[0, \mathrm{~T}] .
\end{aligned}
$$

\subsection{Restrições Lineares}

Podemos exigir que cada solução $\mathrm{x}_{\mathrm{u}}$ da equação $(\mathrm{K})$ satisfaça uma restrição linear do tipo

$$
\mathrm{F}_{\alpha}[\mathrm{x}]=\int_{0}^{\mathrm{T}} \cdot \mathrm{d} \alpha(\mathrm{t}) \cdot \mathrm{x}(\mathrm{t})=\mathrm{z}
$$

sendo $\alpha \in \mathrm{SV}([0, \mathrm{~T}], \mathrm{L}(\mathrm{X}))$ e $\mathrm{z} \in \mathrm{X}$. Vamos mostrar alguns exemplos de tais restrições, exibindo o $\alpha$ correspondente.

1.4.1 Exemplo (Condições de fronteira) Sejam M. N $\in$ L(X. Y). A restrição

$$
M \cdot x(0)-N \cdot x(T)=0
$$


pode ser representada na forma integral $\left(\mathrm{F}_{\alpha}\right)$, com $\mathrm{z}=0$, e $\alpha \in \mathrm{SV}_{0}([0, \mathrm{~T}], \mathrm{L}(\mathrm{X}, \mathrm{Y}))$ dado por

$$
\alpha_{\mathrm{M}, \mathrm{N}}(\mathrm{t}) \cdot \mathrm{x}=\left\{\begin{array}{cl}
0, & \text { se } \mathrm{t}=0 \\
\mathrm{M} \cdot \mathrm{x}, & \text { se } 0<\mathrm{t}<\mathrm{T} \\
(\mathrm{M}-\mathrm{N}) \cdot \mathrm{x}, & \text { se } \mathrm{t}=\mathrm{T}
\end{array}\right.
$$

para todo $\mathrm{x} \in \mathrm{X}$ e todo $\mathrm{t} \in[0, \mathrm{~T}]$.

Realmente, temos que, se

$$
\mathrm{L}[\mathrm{x}]=\mathrm{M} \cdot \mathrm{x}(0)-\mathrm{N} \cdot \mathrm{x}(\mathrm{T})
$$

então $L \in L\left(G_{-}([0, T], X), Y\right)$. Portanto, do Teorema 1.2.1 segue que, para $\mathrm{x} \in \mathrm{X}$ e $0<\mathrm{t} \leq \mathrm{T}$,

$\alpha_{\mathrm{M}, \mathrm{N}}(\mathrm{t}) \cdot \mathrm{x}=\mathrm{L}\left[\chi_{[0, \mathrm{t}]} \cdot \mathrm{x}\right]=\mathrm{M}\left[\left(\chi_{[0, \mathrm{t}]} \cdot \mathrm{x}\right)(0)\right]-\mathrm{N}\left[\left(\chi_{[0, t]} \cdot \mathrm{x}\right)(\mathrm{T})\right]=\left\{\begin{array}{cl}\mathrm{M} \cdot \mathrm{x}, & \text { se } 0<\mathrm{t}<\mathrm{T} \\ (\mathrm{M}-\mathrm{N}) \cdot \mathrm{x}, & \text { se } \mathrm{t}=\mathrm{T}\end{array}\right.$

Considerando $\alpha_{\mathrm{M}, \mathrm{N}}(0)=0$, segue a afirmação.

Observemos que

(a) Se $M \in L(X)$ e $N=I_{X}$ temos

$$
\mathrm{L}[\mathrm{x}(\cdot)]=\mathrm{M} \cdot \mathrm{x}(0)-\mathrm{x}(\mathrm{T})=0,
$$

isto é,

$$
\mathrm{x}(\mathrm{T})=\mathrm{M} \cdot \mathrm{x}(0) .
$$


(b) Se $M \cdot x=\lambda x$ e $N=I_{x}$, temos

$$
\mathrm{L}[\mathrm{x}(\cdot)]=\lambda \mathrm{x}(0)-\mathrm{x}(\mathrm{T})=0
$$

isto é,

$$
\mathrm{x}(\mathrm{T})=\lambda \mathrm{x}(0)
$$

(c) Se $M=N=I_{x}$ temos o caso de ponto de retorno

$$
\mathrm{L}[\mathrm{x}(\cdot)]=\mathrm{x}(0)-\mathrm{x}(\mathrm{T})=0
$$

isto é,

$$
\mathrm{x}(\mathrm{T})=\mathrm{x}(0)
$$

1.4.2 Exemplo (Condições de Periodicidade) A restrição linear

$$
x(s+p)=x(s)
$$

para todo $\mathrm{s} \in[0, \mathrm{~T}]$, e para $\mathrm{p}>0, \mathrm{p} \in \mathbb{R}$, pode ser representada na forma integral $\left(\mathrm{F}_{\alpha}\right)$, $\operatorname{com} \mathrm{z}=0$, onde

$$
\alpha_{\mathrm{s}}(\mathrm{t}) \cdot \mathrm{x}=-\left(\chi_{[\mathrm{s}, \mathrm{s}+\mathrm{p} j} \cdot \mathrm{x}\right)(\mathrm{t})
$$

Realmente, seja, para cada $\mathrm{s} \in[0, \mathrm{~T}]$, 


$$
\mathrm{L}[\mathrm{x}(\cdot)]=\mathrm{x}(\mathrm{p}+\mathrm{s})-\mathrm{x}(\mathrm{s})
$$

Então $\mathrm{L} \in \mathrm{L}\left(\mathrm{G}^{-}([0, \mathrm{~T}], \mathrm{X}), \mathrm{X}\right) \mathrm{e}$

$$
\alpha_{\mathrm{s}}(\mathrm{t}) \cdot \mathrm{x}=\mathrm{L}\left(\chi_{[0, t]} \cdot \mathrm{x}\right)=\left(\chi_{[0, \mathrm{t}]} \cdot \mathrm{x}\right)(\mathrm{s}+\mathrm{p})-\left(\chi_{[0, \mathrm{t}]} \cdot \mathrm{x}\right)(\mathrm{s})
$$

Desse modo,

$$
\alpha_{\mathrm{s}}(\mathrm{t}) \cdot \mathrm{x}=\left\{\begin{array}{cl}
0, & \text { se } \mathrm{t} \in[0, \mathrm{~s}[ \\
-x, & \text { se } \mathrm{t} \in[\mathrm{s}, \mathrm{s}+\mathrm{p}] \\
0, & \text { se } \mathrm{t} \in[\mathrm{s}+\mathrm{p}, \mathrm{T}]
\end{array}\right.
$$

como afirmamos.

1.4.3 Exemplo (Condições Iniciais) Consideremos $\mathrm{z} \in \mathrm{X}$ fixo e $\mathrm{p} \in] 0$, $\mathrm{T}[$. Definindo

$$
\alpha(\mathrm{t}) \cdot \mathrm{y}=\left(\chi_{[\mathbf{p}, \mathbf{T}\}} \cdot \mathrm{y}\right)(\mathrm{t}), \text { para } \mathrm{t} \in[0, \mathrm{~T}] \text { e } \mathrm{y} \in \mathrm{X},
$$

podemos escrever na forma $\left(\mathrm{F}_{\alpha}\right)$, a condição

$$
\mathrm{x}\left(\mathrm{p}_{-}\right)=\mathrm{z}
$$

De fato, se $\mathrm{d}: 0=\mathrm{s}_{0}<\mathrm{s}_{1}<\ldots<\mathrm{s}_{\mathrm{k}}=\mathrm{p}<\ldots<\mathrm{s}_{\mathrm{n}}=\mathrm{T}$, e $\left.\xi_{\mathrm{i}} \in\right] \mathrm{s}_{\mathrm{i}-1} \cdot \mathrm{s}_{\mathrm{i}}[$,

$$
\sum_{\mathrm{i}=1}^{\mathrm{n}}\left[\alpha\left(\mathrm{s}_{\mathrm{i}}\right)-\alpha\left(\mathrm{s}_{\mathrm{i}-1}\right)\right] \cdot \mathrm{x}\left(\xi_{\mathrm{i}}\right)=\left[\alpha\left(\mathrm{s}_{1}\right)-\alpha(0)\right] \cdot \mathrm{x}\left(\xi_{1}\right)+\ldots+\left[\alpha(\mathrm{p})-\alpha\left(\mathrm{s}_{\mathrm{k}-1}\right)\right] \cdot \mathrm{x}\left(\xi_{\mathrm{k}-1}\right)+
$$




$$
+\left[\alpha\left(\mathrm{s}_{\mathrm{k}+1}\right)-\alpha(\mathrm{p})\right] \cdot \mathrm{x}\left(\xi_{\mathrm{k}}\right)+\ldots\left[\alpha\left(\mathrm{s}_{\mathrm{T}}\right)-\alpha\left(\mathrm{s}_{\mathrm{n}-1}\right)\right] \cdot \mathrm{x}\left(\xi_{\mathrm{n}}\right)=\mathrm{x}\left(\xi_{\mathrm{k}-1}\right) .
$$

já que $\alpha\left(\mathrm{s}_{\mathrm{i}}\right)=0$, para $\mathrm{i}=1,2, \ldots \mathrm{k}-1,\left[\alpha\left(\mathrm{s}_{\mathrm{k}+1}\right)-\alpha(\mathrm{k})\right]=0, \mathrm{i}=\mathrm{k}, \mathrm{k}+1 \ldots \mathrm{n} \mathbf{e}$ $\left[\alpha(\mathrm{p})-\alpha\left(\mathrm{s}_{\mathrm{k}-1}\right)\right] \cdot \mathrm{x}\left(\xi_{\mathrm{k}-1}\right)$.

Portanto,

$$
\int_{0}^{\mathrm{T}} \cdot \mathrm{d} \alpha(\mathrm{t}) \cdot \mathrm{x}(\mathrm{t})=\mathrm{x}\left(\mathrm{p}_{-}\right)
$$

Em particular, quando $\mathrm{p}=\mathrm{T}$, isto é,

$$
\alpha(\mathrm{t}) \cdot \mathrm{y}=\left(\chi_{\{\mathrm{T}\}} \cdot \mathrm{y}\right)(\mathrm{t}), \text { para } \mathrm{t} \in[0, \mathrm{~T}] \text { e } \mathrm{y} \in \mathrm{X},
$$

a restrição linear é

$$
\mathrm{x}\left(\mathrm{T}_{-}\right)=\mathrm{z}
$$

\subsection{Equação Adjunta}

Os resultados enunciados a seguir são casos particulares daqueles obtidos em [5] para $\mathrm{t}_{0}=0$. Vamos denotar por $\mathrm{BV}_{0}^{0, T}\left([0, \mathrm{~T}], \mathrm{X}^{\prime}\right)$ o espaço das funções $\mathrm{g}:[0, \mathrm{~T}] \rightarrow \mathrm{X}^{\prime}$ de variação limitada e tais que $g(0)=0$ e $g(T)=g(0)$.

Observemos que, fixados $c \in \mathbb{R}$ e $g \in B V_{0}^{0, T}\left([0, T], X^{\prime}\right)$, os conjuntos 


$$
\mathrm{H}_{\mathrm{g}(\mathrm{s})}(\mathrm{c})=[\mathrm{g}(\mathrm{s}): \mathrm{c}]=\{\mathrm{x} \in \mathrm{X} / \mathrm{g}(\mathrm{s}) \cdot \mathrm{x}=\mathrm{c}\}, \mathrm{s} \in[0, \mathrm{~T}]
$$

são hiperespaços em X.

Como já dissemos na introdução, nosso objeto de estudo são os sistemas governados pela equação $(\mathrm{K})$ e cujas soluções satisfazem a restrição linear $\left(\mathrm{F}_{\alpha}\right)$, isto é, o sistema

$$
\begin{aligned}
& \mathrm{x}(\mathrm{t})-\mathrm{x}_{0}+\int_{0}^{\mathrm{t}} \cdot \mathrm{d}_{\mathrm{s}} \mathrm{K}(\mathrm{t}, \mathrm{s}) \cdot \mathrm{x}(\mathrm{s})=\mathrm{u}(\mathrm{t}), 0 \leq \mathrm{t} \leq \mathrm{T} \\
& \mathrm{F}_{\alpha}[\mathrm{x}]=\int_{0}^{\mathrm{T}} \cdot \mathrm{d} \alpha(\mathrm{t}) \cdot \mathrm{x}(\mathrm{t})=\mathrm{z}
\end{aligned}
$$

$\operatorname{para} \mathrm{K} \in \mathrm{G}_{0}^{\sigma} \mathrm{SV}^{\mathrm{u}}([0, \mathrm{~T}] \times[0, \mathrm{~T}], \mathrm{L}(\mathrm{X})), \alpha \in \mathrm{SV}([0, \mathrm{~T}], \mathrm{L}(\mathrm{X})), \mathrm{z}, \mathrm{x}_{0} \in \mathrm{X}$ e x, u $\in \mathrm{G}([0, \mathrm{~T}], \mathrm{X})$

Em [j] Hönig mostra que o sistema $(\mathrm{K})+\left(\mathrm{F}_{\alpha}\right)$ tem uma equação adjunta dada por

$$
\mathrm{y}(\mathrm{s})+\int_{\mathrm{s}}^{\mathrm{T}} \cdot \mathrm{K}(\mathrm{t}, \mathrm{s})^{*} \cdot \mathrm{d} \mathrm{y}(\mathrm{t})-\int_{0}^{\mathrm{T}} \cdot \mathrm{K}(\mathrm{t}, 0)^{*} \cdot \mathrm{d} \mathrm{y}(\mathrm{t})+\alpha(\mathrm{s})^{*} \cdot \varphi=\mathrm{h}(\mathrm{s}), 0 \leq \mathrm{s} \leq \mathrm{T}
$$

sendo h, $y \in \mathrm{BV}_{0}^{0, T}\left([0, T] . \mathrm{X}^{\prime}\right)$ e $\varphi \in \mathrm{X}^{\prime}$. Observemos que na equação $\left(\mathrm{K}_{\alpha}^{*}\right)$ as integrais tem o significado

$$
<\int_{s}^{\mathrm{T}} \cdot \mathrm{K}(\mathrm{t}, \mathrm{s})^{x} \cdot \mathrm{d} \mathrm{y}(\mathrm{t}), \mathrm{x}>=\lim _{\mathrm{d} \in \mathcal{D}} \sum_{\mathrm{i}=1}^{\mathrm{n}}<\mathrm{K}(\mathrm{s}, \mathrm{T})^{*}\left[\mathrm{y}\left(\mathrm{t}_{\mathrm{i}-1}\right)-\mathrm{y}\left(\mathrm{t}_{\mathrm{i}}\right)\right], \mathrm{x}>=
$$




$$
=\lim _{d \in \mathcal{D}} \sum_{i=1}^{n}<\left[y\left(t_{i-1}\right)-y(i)\right], K(s, T) \cdot x>=\int_{s}^{T} \cdot\langle d y(t), K(s, T) \cdot x>.
$$

Vamos denotar por

$$
\Gamma_{0}=\{(\mathrm{t}, \mathrm{s}) \in[0, \mathrm{~T}] \times[0, \mathrm{~T}] / 0 \leq \mathrm{s} \leq \mathrm{t} \leq \mathrm{T}\}
$$

figura 1.1

Indicamos por $\left(\mathrm{K}_{\alpha}^{*}\right)_{0}$ a equação adjunta $\left(\mathrm{K}_{\alpha}^{*}\right)$ quando $\mathrm{h}=0$. Estamos considerando, em [5], $\mathrm{Y}=\mathrm{X}$ Definimos, para $\alpha \in \mathrm{SV}_{0}([0, \mathrm{~T}], \mathrm{L}(\mathrm{X}))$ e $\mathrm{R} \in \mathrm{G}_{\mathrm{I}}^{\sigma} \cdot \mathrm{SV}^{\mathrm{u}}\left(\Gamma_{0}, \mathrm{~L}(\mathrm{X})\right)$, o operador

$$
\mathrm{J}(0)=\sigma \int_{0}^{\mathrm{T}} \cdot \mathrm{d} \alpha(\mathrm{t}) \cdot \mathrm{R}(\mathrm{t}, 0) \quad[\in \mathrm{L}(\mathrm{X})]
$$

O operador $\mathrm{J}(0)$ fornece condições para garantir a existência de soluções da equação adjunta $\left(\mathrm{K}_{\alpha}^{*}\right)_{0}$. Além disso, tais soluções podem ser expressas em função de $\alpha$ e do resolvente $\mathrm{R}$ no :

1.5.1 Teorema (3.2 de $[5])$ Suponhamos que $\mathrm{K} \in \mathrm{G}_{0}^{\sigma} . \mathrm{SV}^{\mathrm{u}}\left(\Gamma_{0}, \mathrm{~L}(\mathrm{X})\right.$ ) tem um único resolvente $\mathrm{R}$. Dados $\alpha \in \mathrm{SV}_{0}([0, \mathrm{~T}], \mathrm{L}(\mathrm{X}))$ e $\varphi \in \mathrm{X}^{\prime}$, a equação $\left(\mathrm{K}_{\alpha}^{*}\right)_{0}$ tem uma solução $\mathrm{y} \in \mathrm{BV}_{0}^{0, \mathrm{~T}}\left([0, \mathrm{~T}] . \mathrm{X}^{\prime}\right)$ se, e somente se, $\mathrm{J}(0)^{*}{ }_{\hat{\tau}^{*}}=0$. Neste caso, para $0 \leq \mathrm{s} \leq \mathrm{T}$. 


$$
\mathrm{y}(\mathrm{s})=\left\{\int_{\mathrm{s}}^{\mathrm{T}} \cdot \mathrm{d} \alpha(\sigma) \cdot \mathrm{R}(\sigma, \mathrm{s})-\int_{0}^{\mathrm{T}} \cdot \mathrm{d} \alpha(\sigma) \cdot \mathrm{R}(\sigma, 0)\right\}^{*} \varphi
$$

O teorema a seguir nos permitirá caracterizar o conjunto de controles eficazes, em $\mathrm{G}([0, \mathrm{~T}], \mathrm{X})$, para o processo de controle $(\mathrm{K})+\left(\mathrm{F}_{\alpha}\right)$, através das soluções da equação adjunta $\left(\mathrm{K}_{\alpha}^{*}\right)_{0}$.

1.5.2 Teorema (4.1 de [5/) Suponhamos que $\mathrm{K} \in \mathrm{G}_{0}^{\sigma} . \mathrm{SV}^{\mathrm{u}}\left(\Gamma_{0}, \mathrm{~L}(\mathrm{X})\right.$ ) tem um único resolvente $\mathrm{R}$ e $\alpha \in \mathrm{SV}_{0}([0, \mathrm{~T}], \mathrm{L}(\mathrm{X}))$. As seguintes afirmações são equivalentes :

(a) Para $(\mathrm{u}, \mathrm{z}) \in \mathrm{G}([0, \mathrm{~T}], \mathrm{X}) \times \mathrm{X}$, o sistema $(\mathrm{K})+\left(\mathrm{F}_{\boldsymbol{\alpha}}\right)$ tem uma solução $\mathrm{x} \in \mathrm{G}([0, \mathrm{~T}], \mathrm{X})$ se, e somente se, para todo $\operatorname{par}(\mathrm{y}, \varphi) \in \mathrm{BV}_{0}^{0, \mathrm{~T}}\left([0, \mathrm{~T}], \mathrm{X}^{\prime}\right) \times \mathrm{X}^{\prime}$ que satisfaz $\left(\mathrm{K}_{\alpha}^{*}\right)_{0}$ temos

$$
\int_{0}^{\mathrm{T}} \cdot<\mathrm{d} \mathrm{y}(\mathrm{t}), \mathrm{u}(\mathrm{t})>+<\varphi, \mathrm{z}>=0
$$

(b) $J(0) X$ é um subespaço vetorial fechado de X.

1.5.3 Observação A condição (b) é satisfeita, por exemplo, quando tivermos $\operatorname{dim} \mathrm{J}(0) \mathrm{X}<\infty$.

1.5.4 Observação Quando $\mathrm{z}=0 \mathrm{em}(*)$, o termo $\langle\varphi, \mathrm{z}\rangle=0$, para todo $\varphi \in \mathrm{X}^{\prime}$. Como a aplicação

$$
\Psi: \mathrm{u} \in \mathrm{G}([0, \mathrm{~T}], \mathrm{X}) \longmapsto \int_{0}^{\mathrm{T}} \cdot\langle\mathrm{d} \mathrm{y}(\mathrm{t}), \mathrm{u}(\mathrm{t})>\in \mathbb{R}
$$


é linear, segue que $(\star)$ define um subespaço de $\mathrm{G}([0, \mathrm{~T}], \mathrm{X})$, núcleo de $\Psi$. Desse modo. os controles que produzem soluções do sistema $(K)+\left(F_{\alpha}\right)$ são aqueles do núcleo de $\Psi$. naturalmente sempre que a condição (b) do teorema 1.5.2 estiver satisteita. Quando $\mathrm{z} \neq 0$, (*) define um espaço afim em $G([0, T], X)$,

$$
\mathrm{H}_{\Psi}(<\varphi,-\mathrm{z}>)=[\Psi:<\varphi,-\mathrm{z}>]=\{\mathrm{u} \in \mathrm{G}([0, \mathrm{~T}], \mathrm{X}) / \Psi \mathrm{u}=<\varphi,-\mathrm{z}>\}
$$

Tais hiperespaços ajudarão a determinar o conjunto de controles admissíveis para o sistema $(\mathrm{K})+\left(\mathrm{F}_{\alpha}\right)$.

1.5.5 Observação O teorema anterior será essencial para a caracterização dos controles extremais ( Princípio do Máximo ) para o problema de controle que enunciaremos no capítulo 3. O próximo resultado é um caso particular do lema 4.2 de [5] para o caso em que $\mathrm{Y}=\mathbb{R}$ e $\mathrm{t}_{0}=0$.

1.5.6 Lema (4.2 de $[5]) \quad$ Se para $(\mathrm{u}, \xi) \in \mathrm{G}([0, \mathrm{~T}], \mathrm{X}) \times \mathbb{R}$ o sistema $(\mathrm{K})+\left(\mathrm{F}_{\alpha}\right)$ tem uma solução $\mathrm{x} \in \mathrm{G}([0, \mathrm{~T}], \mathrm{X})$ então $\xi=\mathrm{J}(0) \cdot \mathrm{x}_{0}+\xi_{\mathrm{u}}$, sendo

$$
\xi_{\mathrm{u}}=\int_{0}^{\mathrm{T}} \cdot \mathrm{d}_{\sigma}\left[\int_{\sigma}^{\mathrm{T}} \cdot \mathrm{d} \alpha(\tau) \cdot \mathrm{R}(\tau, \sigma)\right] \cdot \mathrm{u}(\sigma)
$$




\section{Capítulo 2}

\section{Controles Admissiveis e o Conjunto de Atingibilidade}

\subsection{Transmissão de Propriedades Geométricas}

No processo de controle governado pela equação $(\mathrm{K})$, as entradas u são as forças externas aplicadas ao sistema com o objetivo de levar o estado inicial $\mathrm{x}_{0}$ a um estado-alvo pré-fixado. As forças u não podem ser tomadas arbitrariamente pois têm limitações naturais, determinadas pela nossa capacidade de produzí-las. Em termos algébricos, tais limitações podem ser expressas através de restrições do tipo

$$
\mathrm{u}(\mathrm{t}) \in \Gamma, 0 \leq \mathrm{t} \leq \mathrm{T}
$$

sendo $\Gamma$ um subconjunto de $X$. Os sistemas com este tipo de restrição no controle são chamados na literatura de sistemas parametrizados. $\mathrm{O}$ conjunto $\Gamma \subset \mathrm{X}$ é chamado na teoria clássica de conjunto restrição. Suas propriedades geométricas induzem propriedades 
semelhantes seja no conjunto de controles admissíveis para o nosso processo de controle, como no conjunto de estados que podem se atingidos com o uso de tais controles. Podemos dizer que as propriedades de $\Gamma$ são transmitidas a estes dois conjuntos. Detalhando : para $\Gamma \subset \mathrm{X}$, consideramos o subconjunto das funções regradas

$$
\mathrm{G}([0, \mathrm{~T}], \Gamma)=\{\mathrm{u} \in \mathrm{G}([0, \mathrm{~T}], \mathrm{X}) / \mathrm{u}(\mathrm{t}) \in \Gamma, 0 \leq \mathrm{t} \leq \mathrm{T}\}
$$

O conjunto de controles admissíveis será tomado como um subconjunto próprio de $\mathrm{G}([0, \mathrm{~T}], \Gamma)$. Nesta seção mostramos que o conjunto $\mathrm{G}([0, \mathrm{~T}], \Gamma)$ herda de $\Gamma$, entre outras, as propriedades de convexidade e fechamento, essenciais para a existência de controle ótimo.

2.1.1 Observação É claro que se $\Lambda \subset \Gamma \subset X$, então

$$
\mathrm{G}([0, \mathrm{~T}], \Lambda) \subset \mathrm{G}([0, \mathrm{~T}], \Gamma)
$$

Em particular, se para $\lambda \in \mathbb{R}, \lambda \geq 0$,

$$
\lambda \Gamma=\{y \in X / y=\lambda x, x \in X\},
$$

então, se $\Gamma$ for convexo e $0 \in \Gamma$,

$$
\begin{gathered}
\mathrm{G}([0, \mathrm{~T}], \lambda \Gamma) \subset \mathrm{G}([0, \mathrm{~T}], \Gamma), \text { para } \lambda, 0 \leq \lambda \leq 1, \\
\mathrm{G}([0, \mathrm{~T}], \lambda \Gamma) \subset \mathrm{G}([0, \mathrm{~T}], \eta \Gamma), \text { para } 1<\lambda<\eta .
\end{gathered}
$$


O Teorema de Hahn-Banach será utilizado no nosso critério para garantir a existência de controles ótimos, o que exige um estudo prévio de como as propriedades geométricas do conjunto $\Gamma$ são transferidas a $G([0, T], \Gamma)$ e deste ao conjunto de controles admissíveis. É o que fazemos na

2.1.2 Proposição Se $\Gamma$ é não-vazio, simétrico, convexo e limitado, então G([0,T], $)$ também é não-vazio, simétrico, convexo e limitado, respectivamente. Além disso, se $0 \in \Gamma$, então $0 \in \mathrm{G}([0, \mathrm{~T}], \Gamma)$.

Prova : Dado $\gamma \in \Gamma$, consideremos o controle constante $\mathrm{u}_{\gamma}(\mathrm{t})=\gamma, \mathrm{t} \in[0, \mathrm{~T}]$. Então $\mathrm{u}_{\gamma} \in \mathrm{G}([0, \mathrm{~T}], \Gamma)$, já que $\mathrm{u}_{\gamma}$ é contínua. Quando $\gamma=0$, temos a última afirmação da proposição. Para a convexidade, sejam $u, v \in G([0, T], \Gamma)$ e $0 \leq \lambda \leq 1$. Então para todo $\mathrm{t} \in[0, \mathrm{~T}]$, segue da convexidade de $\Gamma$ que,

$$
[\lambda \mathbf{u}+(1-\lambda) \mathbf{v}](\mathrm{t})=\lambda \mathbf{u}(\mathrm{t})+(1-\lambda) \mathbf{v}(\mathrm{t}) \in \Gamma .
$$

Além disso, é claro que $[\lambda u+(1-\lambda) v] \in G([0, T], X)$. Portanto, $\lambda u+(1-\lambda) v \in G([0, T], \Gamma)$. Quanto à simetria, se $\mathrm{u} \in \mathrm{G}([0, \mathrm{~T}], \Gamma)$, então $-\mathrm{u} \in \mathrm{G}([0, \mathrm{~T}], \mathrm{X}) \mathrm{e},-\mathrm{u}(\mathrm{t}) \in \Gamma$, para todo $\mathrm{t} \in[0, \mathrm{~T}]$. Logo $-\mathrm{u} \in \mathrm{G}([0, \mathrm{~T}], \Gamma)$. Finalmente, se $\Gamma \subset \mathrm{B}_{\mathrm{r}}(0)$, então sempre que $\mathrm{u} \in \mathrm{G}([0, \mathrm{~T}], \Gamma)$,

$$
\|\mathrm{u}\|=\sup _{\mathrm{t} \in\left[0, \mathrm{~T}^{\prime}\right.}\|\mathrm{u}(\mathrm{t})\| \leq \mathrm{r} .
$$

2.1.3 Proposição Se $\Gamma$ é fechado, então $G([0, T], \Gamma)$ também é fechado.

Prova : Seja $\left(u_{n}\right)_{n \in I N}$ uma sequência de funções em $G([0, T], \Gamma)$ tal que $u_{n} \rightarrow u$ em $\mathrm{G}([0, \mathrm{~T}], \mathrm{X})$. Então, para cada $\mathrm{t} \in[0, \mathrm{~T}[$, existe 


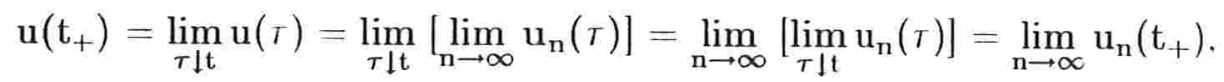

já que $\mathrm{u}_{\mathrm{n}} \rightarrow \mathrm{u}$ uniformemente e $\mathrm{u}_{\mathrm{n}} \in \mathrm{G}([0, \mathrm{~T}], \Gamma), \mathrm{n} \in \mathrm{IN}$. Analogamente, existe

$$
\left.\left.\mathrm{u}\left(\mathrm{t}_{-}\right)=\lim _{\mathrm{n} \rightarrow \infty} \mathrm{u}_{\mathrm{n}}\left(\mathrm{t}_{-}\right), \text {para cada } \mathrm{t} \in\right] 0, \mathrm{~T}\right] .
$$

Logo u é uma função regrada. Além disso, para cada $t \in[0, T]$, temos que $u_{n}(t) \in \Gamma$ e $u_{n}(t) \rightarrow u(t)$. Como $\Gamma$ é fechado, segue que $u(t) \in \Gamma$, para todo $t \in[0, T]$, e assim, $\mathrm{u} \in \mathrm{G}([0, \mathrm{~T}], \Gamma)$.

2.1.4 Proposição Se int $\Gamma \neq \emptyset$, então int $\mathrm{G}([0, \mathrm{~T}], \Gamma) \neq \emptyset$.

Prova : Se int $\Gamma \neq \varnothing$, então existem $\gamma \in \Gamma$ e $r>0$ tais que $B_{r}(\gamma) \subset \Gamma$. Seja $u_{\gamma}(t)=\gamma$, para todo $\mathrm{t} \in[0, \mathrm{~T}]$. Claro que $\mathrm{u}_{\gamma} \in \mathrm{G}([0, \mathrm{~T}], \Gamma)$. Além disso, se

$$
\mathrm{v} \in \mathrm{B}_{\mathrm{r}}\left(\mathrm{u}_{\gamma}\right)=\left\{\mathrm{w} \in \mathrm{G}([0, \mathrm{~T}], \mathrm{X}) /\left\|\mathrm{w}-\mathrm{u}_{\gamma}\right\|<\mathrm{r}\right\},
$$

então v é uma função regrada e

$$
\sup _{t \in[0, T]}\|v(t)-\gamma\|=\left\|v-u_{\gamma}\right\|<r
$$

e portanto, $\|\mathrm{v}(\mathrm{t})-\gamma\|<\mathrm{r}$, isto é, $\mathrm{v}(\mathrm{t}) \in \mathrm{B}_{\mathrm{r}}(\gamma) \subset \Gamma$, para todo $\mathrm{t} \in[0, \mathrm{~T}]$. Temos então que, $v \in G([0, T], \Gamma)$, o que mostra que $B_{r}\left(u_{\gamma}\right) \subset G([0, T], \Gamma)$. Portanto, segue que int $\mathrm{G}([0, \mathrm{~T}], \Gamma) \neq \varnothing$. 
2.1.5 Proposição Suponhamos que int $\Gamma \neq \emptyset$. Então

$$
\mathrm{G}([0, \mathrm{~T}], \text { int } \Gamma) \subset \operatorname{int} \mathrm{G}([0, \mathrm{~T}], \Gamma)
$$

Prova : As proposições 2.1.2 e 2.1.4 garantem que $G([0, T]$, int $\Gamma)$ e int $G([0, T], \Gamma)$ são não-vazios. Suponhamos que $u \in G([0, T]$, int $\Gamma)$. Então existe $r>0$ tal que, para todo $\mathrm{t} \in[0, \mathrm{~T}], \mathrm{B}_{\mathrm{r}}(\mathrm{u}(\mathrm{t})) \subset \Gamma$. Consideremos

$$
\mathrm{B}_{\mathrm{r}}(\mathrm{u})=\{\mathrm{v} \in \mathrm{G}([0, \mathrm{~T}], \mathrm{X}) /\|\mathrm{v}-\mathrm{u}\|<\mathrm{r}\}
$$

Se $\mathrm{v} \in \mathrm{B}_{\mathrm{r}}(\mathrm{u})$, então $\|\mathrm{v}(\mathrm{t})-\mathrm{u}(\mathrm{t})\|<\mathrm{r}$, para todo $\mathrm{t} \in[0, \mathrm{~T}]$. Logo $\mathrm{v}(\mathrm{t}) \in \mathrm{B}_{\mathrm{r}}(\mathrm{u}(\mathrm{t})) \subset \Gamma$, e portanto, $\mathrm{v}(\mathrm{t}) \in \Gamma$, para todo $\mathrm{t} \in[0, \mathrm{~T}]$. Portanto temos que $\mathrm{v} \in \mathrm{G}([0, \mathrm{~T}], \Gamma)$, isto é, $\mathrm{B}_{\mathrm{r}}(\mathrm{u}) \subset \mathrm{G}([0, \mathrm{~T}], \Gamma)$. Logo $\mathrm{u} \in$ int $\mathrm{G}([0, \mathrm{~T}], \Gamma)$.

2.1.6 Exemplo Não é verdade que $\partial \mathrm{G}([0, \mathrm{~T}], \Gamma)=\mathrm{G}([0, \mathrm{~T}], \partial \Gamma)$. De fato, consideremos $\Gamma=[0,1]$ e $\mathrm{u}:[0, \mathrm{~T}] \rightarrow[0,1]$ dada por

$$
\mathrm{u}(\mathrm{t})= \begin{cases}0, & \text { se } 0 \leq \mathrm{t}<\frac{\mathrm{T}}{2} \\ \frac{1}{2}, & \text { se } \mathrm{t}=\frac{\mathrm{T}}{2} \\ 1, & \text { se } \frac{\mathrm{T}}{2}<\mathrm{t} \leq \mathrm{T}\end{cases}
$$

Sejam, para $n \in \mathbb{N}$,

$$
v_{n}(t)=\left\{\begin{array}{cl}
\frac{1}{n+1}, & \text { se } 0 \leq t<\frac{T}{2} \\
\frac{1}{2}-\frac{1}{n+1}, & \text { se } t=\frac{T}{2} \\
1-\frac{1}{n+1}, & \text { se } \frac{T}{2}<t \leq T
\end{array}\right.
$$




$$
W_{n}(t)= \begin{cases}-\frac{1}{n+1}, & \text { se } 0 \leq t<\frac{T}{2}, \\ \frac{1}{2}+\frac{1}{n+1}, & \text { se } t=\frac{T}{2}, \\ 1+\frac{1}{n+1}, & \text { se } \frac{T}{2}<t \leq T .\end{cases}
$$

Desse modo, pela proposição 2.1.5,

$$
\begin{gathered}
\mathrm{v}_{\mathrm{n}} \in \mathrm{G}([0, \mathrm{~T}], \operatorname{int} \Gamma) \subset \operatorname{int} \mathrm{G}([0, \mathrm{~T}], \Gamma), \\
\mathrm{w}_{\mathrm{n}} \notin \mathrm{G}([0, \mathrm{~T}], \Gamma),
\end{gathered}
$$

para todo $n \in \mathbb{N}$. Além disso, como

$$
\left\|\mathrm{v}_{\mathrm{n}}-\mathrm{u}\right\|=\frac{1}{\mathrm{n}}=\left\|\mathrm{w}_{\mathrm{n}}-\mathrm{u}\right\|
$$

dado $r>0$, existe $u m n_{0} \in \mathbb{N}$ suficientemente grande, tal que $v_{n_{0}}, w_{n_{0}} \in B_{r}(u)$. Portanto $u \in \partial \mathrm{G}([0, T], \Gamma)$. No entanto, como $u\left(\frac{T}{2}\right)=\frac{1}{2} \notin\{0,1\}=\partial \Gamma$, segue que $\mathrm{u} \notin \mathrm{G}([0, \mathrm{~T}], \partial \Gamma)$.

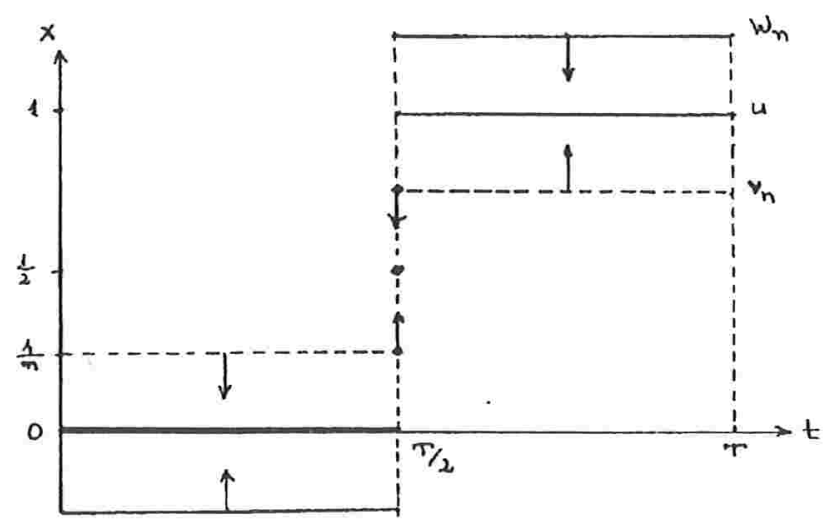

figura 2.1 


\subsection{Controles Eficazes}

Chamaremos de controles eficazes àqueles controles que realizam efetivamente o sistema $(\mathrm{K})+\left(\mathrm{F}_{\alpha}\right)$, dado na seção 1.5 , no sentido de produzirem soluções da equação $(\mathrm{K})$ que cumprem a condição $\left(\mathrm{F}_{\alpha}\right)$. Às soluções associadas a cada controle eficaz chamaremos soluções eficazes.

A existência de uma equação adjunta para o sistema $(K)+\left(F_{\alpha}\right)$ e a caracterização das funções regradas u ( controles ) que produzem soluções eficazes nos permitirá definir um subconjunto de $\mathrm{G}([0, \mathrm{~T}], \mathrm{X})$, onde será permitido escolher os nossos controles. Mais precisamente, vamos fixar aqui, com a ajuda do Teorema 1.5.2, o conjunto dos controles eficazes. Tal conjunto será caracterizado como um subconjunto do núcleo de um funcional linear contínuo definido em $\mathrm{G}([0, \mathrm{~T}], \mathrm{X})$.

É sobre este conjunto de controles que, no capítulo 3, definiremos nosso problema de controle ótimo.

Suponhamos que $\mathrm{K}$ e $\alpha$ estejam nas condições do Teorema 1.5.2, isto é, que $\mathrm{K} \in \mathrm{G}_{0}^{\sigma} \cdot \mathrm{SV}^{\mathrm{u}}\left(\Gamma_{0}, \mathrm{~L}(\mathrm{X})\right)$ tenha um único resolvente $\mathrm{R}, \alpha \in \mathrm{SV}_{0}([0, \mathrm{~T}], \mathrm{L}(\mathrm{X}))$ e que além disso,

$\mathrm{J}(0) \mathrm{X}$ é um subespaço vetorial fechado de X,

sendo $\mathrm{J}(0)$ o operador definido em (1.24).

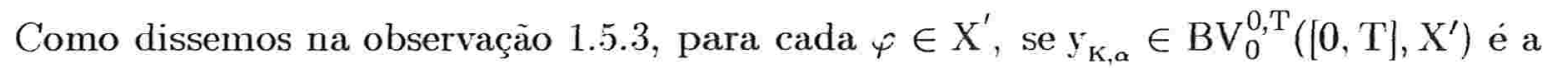
solução de $\left(\mathrm{K}_{\alpha}^{*}\right)_{0}$, fica definida a aplicação linear 


$$
\Psi_{\mathrm{K}, \boldsymbol{\alpha}}: \mathrm{u} \in \mathrm{G}([0, \mathrm{~T}], \mathrm{X}) \longmapsto \int_{0}^{\mathrm{T}} \cdot\left\langle\mathrm{d} \mathrm{y}_{\mathrm{K}, \alpha}(\mathrm{t}), \mathrm{u}(\mathrm{t})>\in \mathbb{R}\right.
$$

Temos também que $\Psi_{\mathrm{K}, \boldsymbol{\alpha}}$ é contínua, pois $\mathrm{BV}_{0}^{0, \mathrm{~T}}\left([0, \mathrm{~T}], \mathrm{X}^{\prime}\right)=\mathrm{SV}_{0}^{0, \mathrm{~T}}\left([0, \mathrm{~T}], \mathrm{X}^{\prime}\right)$ e, pelo Teorema 1.1.9,

$$
\left\|\Psi_{\mathrm{K}, \alpha}(\mathrm{u})\right\|=\mathrm{SV}\left[\mathrm{y}_{\mathrm{K}, \alpha}\right]\left\|\mathrm{u}^{-}\right\|
$$

A aplicação $\Psi_{K, \alpha}$ leva à definição dos conjuntos :

$$
\begin{gathered}
\mathcal{U}_{\mathrm{K}, \alpha}=\operatorname{Ker} \Psi_{\mathrm{K}, \alpha} . \\
\mathcal{U}_{\mathrm{K}, \alpha, \Gamma}=\mathcal{U}_{\mathrm{K}, \alpha} \cap \mathrm{G}([0, \mathrm{~T}], \Gamma) .
\end{gathered}
$$

\section{Temos então que}

- $\mathcal{U}_{\mathrm{K}, \boldsymbol{\alpha}}$ é um subespaço fechado de $\mathrm{G}([0, \mathrm{~T}], \mathrm{X})$, de codimensão 1 . De fato, seja $\mathrm{u}_{\mathrm{n}} \rightarrow \mathrm{u}$ em $\mathrm{G}([0, \mathrm{~T}], \mathrm{X})$ tal que $\mathrm{u}_{\mathrm{n}} \in \mathcal{U}_{\mathrm{K}, \alpha},(\mathrm{n} \in \mathrm{N})$. Como $\Psi_{\mathrm{K}, \alpha}$ é contínua, $\Psi_{\mathrm{K}, \alpha}\left(\mathrm{u}_{\mathrm{n}}\right) \rightarrow \Psi_{\mathrm{K}, \alpha}(\mathrm{u})$, isto é,

$$
\Psi_{K, \alpha}(u)=\lim _{n \rightarrow \infty} \Psi_{K . \alpha}\left(u_{n}\right)=0
$$

Portanto $\mathrm{u} \in \mathcal{U}_{\mathrm{K}, \alpha}$.

$\cdot \mathcal{U}_{\mathrm{K}, \alpha, \Gamma}$ é o subconjunto de $\mathcal{U}_{\mathrm{K}, \alpha}$ das funções regradas $u:[0, \mathrm{~T}] \rightarrow \mathrm{X}$ que têm valor em $\Gamma$. 
- Se u $\in \mathcal{U}_{\mathrm{K}, \alpha, \Gamma}$, então o sistema $(\mathrm{K})+\left(\mathrm{F}_{\alpha}\right), \operatorname{com} z=0$ (v. seção 1.5) tem uma solução $\mathrm{x}_{\mathrm{u}} \in \mathrm{G}([0, \mathrm{~T}], \mathrm{X})$.

2.2.1 Observação Quando $\mathrm{z} \neq 0$, definimos os hiperespaços

$$
\mathcal{U}_{\mathrm{K}, \boldsymbol{\alpha}, \mathrm{z}}=\left\{\mathrm{u} \in \mathrm{G}([0, \mathrm{~T}], \mathrm{X}) / \Psi_{\mathrm{K}, \alpha}(\mathrm{u})=<\varphi, \mathrm{z}>\right\}
$$

Sendo assim, podemos considerar o conjunto

$$
\mathcal{U}_{\mathrm{K}, \alpha, z, \Gamma}=\mathcal{U}_{\mathrm{K}, \alpha, z} \cap \mathrm{G}([0, \mathrm{~T}], \Gamma)
$$

e garantir que quando $\mathrm{u} \in \mathcal{U}_{\mathrm{K}, \boldsymbol{\alpha}, \mathrm{z}, \mathrm{\Gamma}}$ o sistema $(\mathrm{K})+\left(\mathrm{F}_{\boldsymbol{\alpha}}\right)$ tem uma solução $\mathrm{x}_{\mathrm{u}} \in \mathrm{G}([0, \mathrm{~T}], \mathrm{X})$.

Com o intuito de não carregar a notação : já que $\mathrm{K}$ e $\alpha$ estão fixados, doravante denotaremos

$$
\mathcal{U}_{\mathrm{K}, \alpha}=\mathcal{U}, \quad \mathcal{U}_{\mathrm{K}, \alpha, \Gamma}=\mathcal{U}_{\Gamma} \quad \text { e } \mathcal{U}_{\mathrm{K}, \alpha, z, \Gamma}=\mathcal{U}_{\mathrm{z}, \Gamma}
$$

e vamos nos referir a $\mathcal{U}_{\Gamma}$ e $\mathcal{U}_{z, \Gamma}$ como o Conjunto dos Controles Admissíveis (relativos ao sistema $(\mathrm{K})+\left(\mathrm{F}_{\alpha}\right), \operatorname{com} \mathrm{z}=0 \mathrm{e} \mathrm{z} \neq 0$, respectivamente). Claro que a escolha do conjunto $\Gamma$ determina as propriedades geométricas de $\mathcal{U}_{\Gamma}$ e $\mathcal{U}_{z, \Gamma}$. Vamos denotar o subconjunto de $\mathcal{U}_{\Gamma}$ das funções regradas contínuas à esquerda por $\mathcal{U}_{\Gamma}^{-}$, isto é

$$
\mathcal{U}_{\Gamma}^{-}=\mathcal{U} \cap \mathrm{G}^{-}([0, \mathrm{~T}], \Gamma)
$$


Vamos a seguir detalhar algumas propriedades de $\mathcal{U}_{\Gamma}$ que são herdadas do conjunto restrição $\Gamma$, já que os efeitos no nosso sistema serão sempre provocados por forças em $\mathcal{U}_{\Gamma}$.

2.2.2 Proposição Se $\gamma \in \Gamma$, então os controles constantes $\mathrm{u}_{\gamma}(\mathrm{t})=\gamma$, $\mathrm{t} \in[0, \mathrm{~T} \mid$, pertencem a $\mathcal{U}_{\Gamma}$.

Prova : Seja $\gamma \in \Gamma$, e consideremos o controle constante $\mathrm{u}_{\gamma}$. É claro que $\mathrm{u}_{\gamma} \in \mathrm{G}([0, \mathrm{~T}], \Gamma)$. Além disso, como $\mathrm{u}_{\gamma}$ é constante (ver exemplo 1.1.11) e $\mathrm{y}_{\mathrm{K}, \alpha} \in \mathrm{BV}_{0}^{0, \mathrm{~T}}\left([0, \mathrm{~T}], \mathrm{X}^{\prime}\right)$, isto é. $\mathrm{y}_{\mathrm{K}, \alpha}(\mathrm{T})=\mathrm{y}_{\mathrm{K}, \alpha}(0)=0$,

$$
\int_{0}^{\mathrm{T}} \cdot<\operatorname{dy}(\mathrm{t}), \mathrm{u}_{\gamma}(\mathrm{t})>=<\mathrm{y}_{\mathrm{K}, \alpha}(\mathrm{T})-\mathrm{y}_{\mathrm{K}, \alpha}(0), \mathrm{u}_{\gamma}(\mathrm{t})>=0
$$

Assim, $\mathrm{u}_{\gamma} \in \mathcal{U}$, e portanto, $\mathrm{u}_{\gamma} \in \mathcal{U}_{\Gamma}$.

2.2.3 Proposição $\mathcal{U}_{\Gamma}$ é não-vazio e simétrico se, e somente se, o conjunto restrição $\Gamma$ é não-vazio e simétrico.

Prova : Se $\Gamma \neq \emptyset$, consideremos o controle $\mathrm{u}_{\gamma}$, com $\gamma \in \Gamma$. Pelo Lema anterior, $\mathrm{u}_{\gamma} \in \mathcal{U}_{\Gamma}$, e portanto $\mathcal{U}_{\Gamma} \neq \varnothing$. Reciprocamente, se $\mathcal{U}_{\Gamma} \neq \varnothing$ então existe $\mathrm{u} \in \mathrm{G}([0, \mathrm{~T}], \Gamma)$, e portanto. existe $t_{0} \in[0, T]$ tal que $u\left(t_{0}\right) \in \Gamma$. Logo $\Gamma \neq \varnothing$. Suponhamos agora que $\mathcal{U}_{\Gamma}$ é simétrico. Se $\gamma \in \Gamma$, então, pelo Lema anterior, $\mathrm{u}_{\gamma} \in \mathcal{U}_{\Gamma}$. Logo $\mathrm{u}_{-\gamma} \in \mathcal{U}_{\Gamma}$, ou seja, $-\gamma \in \Gamma$ e portanto $\Gamma$ é simétrico. Por outro lado, se $\Gamma$ é simétrico, seja $u \in \mathcal{U}_{\Gamma}$. Então, para cada $\mathrm{t} \in[0, \mathrm{~T}], \mathrm{u}(\mathrm{t}) \in \Gamma$, e portanto $-\mathrm{u}(\mathrm{t}) \in \Gamma$, para $\mathrm{t} \in[0, \mathrm{~T}]$, isto é, $-\mathrm{u} \in \mathrm{G}([0, \mathrm{~T}], \Gamma)$, já que $-\mathrm{u} \in \mathrm{G}([0, \mathrm{~T}], \mathrm{X})$. Logo $\mathcal{U}_{\Gamma}$ é simétrico.

2.2.4 Proposição Se $\Gamma \subset \mathrm{X}$ é um conjunto convexo e limitado, então $\mathcal{U}_{\Gamma}$ também é convexo e limitado.

Prova : $\mathcal{U}_{\Gamma}$ herda de $\Gamma$. via Proposição 2.1.2. a convexidade e a limitaçào, já que 
$\mathrm{G}([0, \mathrm{~T}], \Gamma)$ é convexo e limitado e $\mathcal{U}_{\Gamma}=\operatorname{Ker} \Psi_{K, \boldsymbol{\alpha}} \cap \mathrm{G}([0, \mathrm{~T}], \Gamma)$.

2.2.5 Observação Segue da Observação 2.1.1 que, se $\Lambda, \Gamma \subset \mathrm{X}$ e $\Lambda \subset \Gamma$, então

$$
\mathcal{U}_{\Lambda} \subset \mathcal{U}_{\Gamma}
$$

Em particular, como será necessário estudar a potência dos controles adequada para se atingir um estado pré-definido, temos

$$
\begin{gathered}
\mathcal{U}_{\lambda \Gamma} \subset \mathcal{U}_{\Gamma}, \text { para cada } \lambda, 0 \leq \lambda \leq 1 . \\
\mathcal{U}_{\lambda \Gamma} \subset \mathcal{U}_{\eta \Gamma}, 1 \leq \lambda<\eta .
\end{gathered}
$$

2.2.6 Proposição Se $\Gamma \subset \mathrm{X}$ é fechado, então $\mathcal{U}_{\Gamma}$ também é fechado.

Prova : Se $\Gamma$ é fechado, então (ver Proposição 2.1.3) G([0,T], Г) também o é. Lembrando que $\mathcal{U}$ é um subespaço de $\mathrm{G}([0, \mathrm{~T}], \mathrm{X})$, segue que $\mathcal{U}_{\Gamma}$ é fechado.

2.2.7 Observação $\quad$ Se $u_{0}(t)=0$, para $t \in[0, T]$, entào

$$
\mathrm{u}_{0} \in \mathcal{U}_{\Gamma} \text { se, e somente se, } 0 \in \Gamma \text {. }
$$

Observemos agora que int $\mathcal{U}_{\Gamma}=\emptyset$, já que $\mathcal{U}_{\Gamma}$ está contido no subespaço próprio $\mathcal{U}$ de $\mathrm{G}([0, \mathrm{~T}], \mathrm{X})$. Para evitar a imposição de condições de transversalidade entre os núcleos de 
funcionais definidos em $X$ e o conjunto dos estados que podem ser atingidos com o uso do controles em $\mathcal{U}_{\Gamma}$, vamos considerar

2.2.8 Definição Definimos o interior relativo e fronteira relativa de $\mathcal{U}_{\Gamma}$ por

$$
\begin{gathered}
\text { int }_{\text {rel }} \mathcal{U}_{\Gamma}=\mathcal{U} \cap \text { int } \mathrm{G}([0, \mathrm{~T}], \Gamma) . \\
\partial_{\text {rel }} \mathcal{U}_{\Gamma}=\mathcal{U} \cap \partial \mathrm{G}([0, \mathrm{~T}], \Gamma) .
\end{gathered}
$$

2.2.9 Proposição Se int $\Gamma \neq \varnothing$, então int ${ }_{\text {rel }} \mathcal{U}_{\Gamma} \neq \varnothing$.

Prova : Basta lembrarmos que se, int $\Gamma \neq \varnothing$, então, pela proposição 2.1 .4 , temos que int $\mathrm{G}([0, \mathrm{~T}], \Gamma) \neq \varnothing$.

2.2.10 Proposição Suponhamos que int $\Gamma \neq \varnothing$. Então

$$
\left[\mathcal{U}_{\Gamma} \mid=\mathcal{U}\right.
$$

sendo que $\left[\mathcal{U}_{\Gamma}\right]$ é o subespaço de $\mathrm{G}([0, \mathrm{~T}], \mathrm{X})$ gerado por $\mathcal{U}_{\Gamma}$.

Prova : Como $\mathcal{U}$ é um subespaço fechado de $\mathrm{G}([0, \mathrm{~T}], \mathrm{X})$ e $\mathcal{U}_{\Gamma} \subset \mathcal{U}$, segue que $\left[\mathcal{U}_{\Gamma}\right] \subset \mathcal{U}$. Por outro lado, segue da Proposição anterior que int ${ }_{\mathrm{rel}} \mathcal{U}_{\mathrm{r}} \neq \varnothing$. Sejam então $\mathrm{v} \in \operatorname{int} \mathcal{U}_{\mathrm{r}}$ e $\mathrm{B}_{\epsilon}(\mathrm{v}) \subset \mathcal{U}_{\Gamma}$, para $\epsilon>0$ suficientemente pequeno. Dado $\mathrm{u} \in \mathcal{U}$, consideremos o vetor $\mathrm{u}-\mathrm{v}$. Então existem $\mathrm{w} \in \mathrm{B}_{\epsilon}(\mathrm{v})$ e $\lambda \in \mathbb{R}$ tais que $\mathrm{u}-\mathrm{v}=\lambda \mathrm{w}$. Portanto, $\mathrm{u}=\mathrm{v}+\lambda \mathrm{w}$. com $\mathrm{v}, \mathrm{w} \in \mathrm{B}_{\epsilon}(\mathrm{v}) \subset \mathcal{U}_{\mathrm{r}}$. Logo $\mathrm{u} \in\left[\mathcal{U}_{\mathrm{r}}\right]$ e $\mathcal{U} \subset\left[\mathcal{U}_{\mathrm{r}}\right]$. 
É importante conhecer propriedades sobre aquelas forças de maior potência que podem ser utilizadas em uma certa direção a cada instante. Como $\mathcal{U}_{\Gamma}$ é convexo e limitado (para $\Gamma$ convexo e limitado) é de se esperar que tais forças estejam localizadas na fronteira relativa de $\mathcal{U}_{\Gamma}$ e que estejam relacionadas, claro, com os extremos do conjunto restrição $\Gamma$. Em geral, os controles ótimos $\mathrm{u}^{*}$ associados aos funcionais custo têm tais propriedades extremais e as respostas ótimas $\mathrm{x}_{\mathrm{u}^{*}}$ associadas a eles têm seu valor $\mathrm{x}_{\mathrm{u}^{*}}(\mathrm{t})$ na fronteira do conjunto dos estados que podem ser atingidos num instante $t$.

\subsubsection{Proposição $\quad \mathrm{u}_{\gamma} \in \partial_{\text {rel }} \mathcal{U}_{\Gamma}$ se, e somente se, $\gamma \in \partial \Gamma$.}

Prova : Suponhamos que $\gamma \in \partial \Gamma$. Então, para todo $r>0$, existem $\gamma_{1}, \gamma_{2} \in B_{r}(\gamma)$ tais que $\gamma_{1} \in \Gamma$ e $\gamma_{2} \notin \Gamma$. Sejam $u_{1}, u_{2}:[0, T] \rightarrow X$, dadas por $\mathrm{u}_{\mathrm{i}}(\mathrm{t})=\gamma_{\mathrm{i}}$, para $\mathrm{t} \in[0, \mathrm{~T}]$. Claro que, como $\mathrm{u}_{1}$ e $\mathrm{u}_{2}$ são constantes, segue que $\mathrm{u}_{1}, \mathrm{u}_{2} \in \mathcal{U}$. Além disso, $\gamma_{1} \in \Gamma$ e $\gamma_{2} \notin \Gamma$ acarretam, respectivamente, que $\mathrm{u}_{1} \in \mathrm{G}([0, \mathrm{~T}], \Gamma)$ e $\mathrm{u}_{2} \notin \mathrm{G}([0, \mathrm{~T}], \Gamma)$. Portanto,

. $\mathrm{u}_{1} \in \mathcal{U}_{\Gamma}$.

. $\mathrm{u}_{2} \in \mathcal{U} \operatorname{mas} \mathrm{u}_{2} \notin \mathcal{U}_{\Gamma}$

. $\mathrm{u}_{1}, \mathrm{u}_{2} \in \mathrm{B}_{\mathrm{r}}\left(\mathrm{u}_{\gamma}\right) \cap \mathcal{U}$. pois

$$
\left\|\mathrm{u}_{\mathrm{i}}-\mathrm{u}_{\gamma}\right\|=\sup _{0 \in[0, T]}\left\|\mathrm{u}_{\mathrm{i}}(\mathrm{t})-\mathrm{u}_{\gamma}(\mathrm{t})\right\|=\left\|\gamma_{\mathrm{i}}-\gamma\right\|<\mathrm{r} .
$$

Logo, $\mathrm{u}_{\gamma} \in \partial_{\text {rel }} \mathcal{U}_{\Gamma}$.

Por outro lado, se $u_{\gamma} \in \partial_{\text {rel }} \mathcal{U}_{\Gamma}$, então existem $v, w \in G([0, T], X)$ tais que . $\mathrm{v} \in \mathcal{U}_{\Gamma}$.

$\mathrm{w} \in \mathcal{U}$ e w $\notin \mathrm{G}([0, \mathrm{~T}], \Gamma)$ 
. $\mathbf{v}, \mathbf{w} \in \mathrm{B}_{\mathrm{r}}\left(\mathrm{u}_{\gamma}\right)$, para todo $\mathrm{r}>0$.

Como $w \in G([0, T], X) \backslash G([0, T], \Gamma)$, segue que existe $t_{0} \in[0, T]$ tal que $w\left(t_{0}\right) \notin \Gamma$. Logo, para todo $\mathrm{r}>0$, existem $\gamma_{1}=\mathrm{v}\left(\mathrm{t}_{0}\right) \in \Gamma$, e $\gamma_{2}=\mathrm{w}\left(\mathrm{t}_{0}\right) \notin \Gamma$ tais que

$$
\begin{aligned}
& \left\|\gamma_{1}-\gamma\right\|=\left\|\mathrm{v}\left(\mathrm{t}_{0}\right)-\mathrm{u}_{\gamma}\left(\mathrm{t}_{0}\right)\right\| \leq \sup _{\mathrm{t} \in[0, \mathrm{~T}]}\left\|\mathrm{v}(\mathrm{t})-\mathrm{u}_{\gamma}(\mathrm{t})\right\|=\left\|\mathrm{v}-\mathrm{u}_{\gamma}\right\|<\mathrm{r}, \\
& \left\|\gamma_{2}-\gamma\right\|=\left\|\mathrm{w}\left(\mathrm{t}_{0}\right)-\mathrm{u}_{\gamma}\left(\mathrm{t}_{0}\right)\right\| \leq \sup _{\mathrm{t} \in[0, \mathrm{~T}]}\left\|\mathrm{w}(\mathrm{t})-\mathrm{u}_{\gamma}(\mathrm{t})\right\|=\left\|\mathrm{w}-\mathrm{u}_{\gamma}\right\|<\mathrm{r} .
\end{aligned}
$$

Portanto, $\gamma \in \partial \Gamma$.

\subsection{O Conjunto de Atingibilidade}

Como já observamos na seção anterior, a cada $u \in \mathcal{U}_{\Gamma}$, o conjunto dos controles admissíveis, corresponde uma solução do sistema $(\mathrm{K})+\left(\mathrm{F}_{\alpha}\right)$, dada pela fórmula $(\rho)(\mathrm{v}$. seção 1.3$)$. Portanto, para cada instante fixado $\overline{\mathrm{t}} \in[0, \mathrm{~T}]$, o estado do sistema neste instante, $\mathrm{x}_{\mathrm{u}}(\overline{\mathrm{t}}) \in$ $\mathrm{X}$, é dado por

$$
\mathrm{x}_{\mathrm{u}}(\overline{\mathrm{t}})=\mathrm{u}(\overline{\mathrm{t}})+\mathrm{R}(\overline{\mathrm{t}}, 0) \cdot \mathrm{x}_{0}-\int_{0}^{\overline{\mathrm{t}}} \cdot \mathrm{d}_{\tau} \mathrm{R}(\overline{\mathrm{t}}, \tau) \cdot \mathrm{u}(\tau)
$$

Claro que mudanças nos controles $u$, com $\bar{t}$ fixo, acarretam alterações no estado $x_{u}(\bar{t})$. $\mathrm{O}$ conjunto dos pontos do espaço $\mathrm{X}$, que podem ser alcançados a partir do estado inicial $\mathrm{x}_{0}$, utilizando-se controles $\mathrm{u} \in \mathcal{U}_{\Gamma}$, desempenha, junto com suas propriedades geométricas, um papel essencial na Teoria do Controle em Tempo Ótimo. Nosso objetivo aqui é mostrar que tal conjunto herda algumas propriedades geométricas do conjunto restrição $\Gamma$.

Consideremos a seguinte aplicação 


$$
\mathcal{F}: \mathrm{G}([0, \mathrm{~T}], \mathrm{X}) \rightarrow \mathrm{G}([0, \mathrm{~T}], \mathrm{X})
$$

definida por

$$
(\mathcal{F} \mathrm{u})(\mathrm{t})=\mathrm{u}(\mathrm{t})-\int_{0}^{\mathrm{t}} \cdot \mathrm{d}_{\tau} \mathrm{R}(\mathrm{t}, \tau) \cdot \mathrm{u}(\tau), 0 \leq \mathrm{t} \leq \mathrm{T}
$$

sendo $R \in G_{I}^{\sigma} \cdot S V^{u}$ o único resolvente de $K \in G_{0}^{\sigma} \cdot S V^{u}$.

2.3.1 Observação Observemos em primeiro lugar que $\mathcal{F}$ é uma aplicaçāo linear. Além disso, $\mathcal{F} \mathrm{u}=\mathrm{x}_{\mathrm{u}}-\mathrm{R}(\cdot, 0) \cdot \mathrm{x}_{0}$ e portanto $\mathcal{F}$ depende continuamente de $\mathrm{u}$ ( ver Observação 1.3.4).

Usando a função $\mathcal{F}$, o processo de controle para a equação $(K)$, definido pela fórmula $(\rho)$, pode ser visto como um processo de controle afim "perturbado ",

$$
\mathrm{u} \in \mathrm{G}([0, \mathrm{~T}], \mathrm{X}) \rightarrow \Phi(\mathrm{u})(\mathrm{t})=(\mathcal{F} \mathrm{u})(\mathrm{t})+\mathrm{X}_{0} \in \mathrm{X}
$$

sendo o termo "perturbante $" \mathrm{X}_{0} \in \mathrm{X}$ dado por

$$
\mathrm{X}_{0}=\left\{\mathrm{R}(\mathrm{t}, 0) \cdot \mathrm{x}_{0}\right\}
$$

para $\mathrm{x}_{0} \in \mathrm{X}$ fixo.

Denotaremos por $\mathcal{W}_{\mathrm{K}}\left(\mathrm{t}, 0 . \mathrm{x}_{0}, \mathrm{U}\right)$ o Conjunto dos Pontos Atingiveis, a partir de $\mathrm{x}_{0}$, no instante t. e usando controles num conjunto $\mathrm{U} \subset \mathrm{G}([0, \mathrm{~T}], \mathrm{X})$. Ou seja, 


$$
\begin{gathered}
\mathcal{W}_{\mathrm{K}}\left(\mathrm{t}, 0, \mathrm{x}_{0}, \mathrm{U}\right)=\left\{(\mathcal{F} \mathrm{u})(\mathrm{t})+\mathrm{R}(\mathrm{t}, 0) \cdot \mathrm{x}_{0} / \mathrm{u} \in \mathrm{U}\right\}= \\
=\{(\mathcal{F} \mathrm{u})(\mathrm{t}) / \mathrm{u} \in \mathrm{U}\}+\mathrm{R}(\mathrm{t}, 0) \cdot \mathrm{x}_{0}=(\mathcal{F} \mathrm{U})(\mathrm{t})+\mathrm{R}(\mathrm{t}, 0) \cdot \mathrm{x}_{0},
\end{gathered}
$$

sendo $\mathrm{R}$ o resolvente (único) de $\mathrm{K} \in \mathrm{G}_{0}^{\sigma} \cdot \mathrm{SV}^{\mathrm{u}}([0, \mathrm{~T}] \times[0, \mathrm{~T}], \mathrm{L}(\mathrm{X}))$ e $\mathrm{x}_{0} \in \mathrm{X}$ fixo.

\subsubsection{Observação Sejam $t \in[0, T]$ e $x_{0} \in X$.}

(a) Se $\mathrm{U} \subset \mathrm{G}([0, \mathrm{~T}], \mathrm{X})$, então $\mathcal{W}_{\mathrm{K}}\left(\mathrm{t}, 0, \mathrm{x}_{0}, \mathrm{U}\right) \subset \mathrm{X}$ é uma translação rígida de $\mathcal{W}_{\mathrm{K}}(\mathrm{t}, 0,0, \mathrm{U})$ pelo vetor $\mathrm{R}(\mathrm{t}, 0) \cdot \mathrm{x}_{0}$. Sua geometria portanto, independe de $\mathrm{R}(\mathrm{t}, 0) \cdot \mathrm{x}_{0}$. que só é importante para sua localização em X.

(b) Se U é um subespaço $G([0, T], X)$, então $\mathcal{W}\left(t, 0, x_{0}, U\right)$ é uma variedade linear de $X$. Em particular, se $\mathrm{x}_{0} \in \operatorname{Ker} \mathrm{R}(\mathrm{t}, 0)$, então $\mathcal{W}\left(\mathrm{t}, 0, \mathrm{x}_{0}, \mathrm{U}\right)$ é um subespaço de $\mathrm{X}$.

2.3.3 Notação Como o núcleo $\mathrm{K}$ e o instante inicial $\mathrm{t}_{0}=0$ estão fixados, vamos usar a seguinte notação :

$$
\mathcal{W}\left(\mathrm{t}, \mathrm{x}_{0}, \mathrm{U}\right)=\mathcal{W}_{\mathrm{K}}\left(\mathrm{t}, 0 . \mathrm{x}_{0}, \mathrm{U}\right) \text { e } \mathcal{W}(\mathrm{t}, 0, \mathrm{U})=\mathcal{W}_{\mathrm{K}}(\mathrm{t}, 0.0, \mathrm{U})
$$

2.3.4 Observação Da observação 2.3.2(a) temos que

$$
\mathcal{W}\left(\mathrm{t}, \mathrm{x}_{0}, \mathrm{U}\right)=\mathcal{W}(\mathrm{t}, 0, \mathrm{U})+\mathrm{R}(\mathrm{t}, 0) \cdot \mathrm{x}_{0}
$$


Claro que

$$
\mathrm{x}_{0} \in \operatorname{Ker} \mathrm{R}(\mathrm{t}, 0) \Longrightarrow \mathcal{W}\left(\mathrm{t}, \mathrm{x}_{0}, \mathrm{U}\right)=\mathcal{W}(\mathrm{t}, 0, \mathrm{U})
$$

Nas próximas proposições vamos estudar as propriedades geométricas do conjunto dos estados atingiveis .

2.3.5 Proposição Sejam $t \in[0, T]$ e $x_{0} \in X$. Então

$$
0 \in \mathcal{W}\left(\mathrm{t}, \mathrm{x}_{0}, \mathbf{U}\right) \text { se, e somente se, } \mathrm{u}_{-2 \mathbf{x}_{\mathbf{0}}} \in \mathbf{U}
$$

Prova : Temos que $0 \in \mathcal{W}\left(\mathrm{t}, \mathrm{x}_{0}, \mathrm{U}\right)$ se, e somente se, existe um controle $\mathrm{u} \in \mathrm{U}$ tal que $(\mathcal{F} \mathrm{u})(\mathrm{t})+\mathrm{R}(\mathrm{t}, 0) \cdot \mathrm{x}_{0}=0$, isto é,

$$
(\mathcal{F} \mathrm{u})(\mathrm{t})=-\mathrm{R}(\mathrm{t}, 0) \cdot \mathrm{x}_{0} .
$$

Consideremos $\mathrm{u}(\mathrm{t})=-2 \mathrm{x}_{0}$, para $\mathrm{t} \in[0, \mathrm{~T}]$. $\mathrm{O}$ exemplo 1.3.5 garante que tal controle produz a solução

$$
\mathrm{x}_{-2 \mathrm{x}_{0}}(\mathrm{t})=\mathrm{R}(\mathrm{t}, 0) \cdot\left(-2 \mathrm{x}_{0}+\mathrm{x}_{0}\right)=-\mathrm{R}(\mathrm{t}, 0) \cdot \mathrm{x}_{0} \text {, para } \mathrm{t} \in[0, \mathrm{~T}] .
$$

Portanto, acontece (2.22) se, e somente se, $\mathrm{u}_{-2 \mathrm{x}_{0}} \in \mathbf{U}$.

2.3.6 Observação Quando $\mathbf{U}=\mathcal{U}_{\Gamma}$, com $\Gamma$ simétrico, temos que 


$$
0 \in \mathcal{W}\left(\mathrm{t}, \mathrm{x}_{0}, \mathcal{U}_{\Gamma}\right) \text { se, e somente se, } \mathrm{x}_{0} \in \frac{1}{2} \Gamma
$$

já que $\mathrm{u}_{-2 \mathrm{x}_{0}} \in \mathcal{U}_{\Gamma}$ se, e somente se, $-2 \mathrm{x}_{0} \in \Gamma$ (ver Proposição 2.2.3).

\subsubsection{Proposição Sejam $t \in[0, T]$ e $x_{0} \in X$. Então}

$$
\mathcal{W}\left(\mathrm{t}, \mathrm{x}_{0}, \mathcal{U}_{\mathrm{r}}\right) \neq \emptyset \text {, se, e somente se, } \Gamma \neq \varnothing
$$

Prova : Da definição do conjunto de atingibilidade temos que $\mathcal{W}\left(\mathrm{t}, \mathrm{x}_{0}, \mathrm{U}\right) \neq \varnothing$ se, e somente se, $\mathbf{U} \neq \varnothing$. Quando $\mathbf{U}=\mathcal{U}_{\Gamma}$, a Proposição 2.2.3 dá o resultado.

2.3.8 Proposição Suponhamos que $x_{0} \in \operatorname{Ker} R(t, 0)$. Então, para cada $t \in[0, T]$,

$$
\mathcal{W}\left(\mathrm{t}, \mathrm{x}_{0}, \mathcal{U}_{\Gamma}\right) \text { é simétrico se, e somente se, } \Gamma \text { é simétrico. }
$$

Prova : Segue da linearidade da função $\mathcal{F}$ e da proposição 2.2.3.

2.3.9 Proposição Sejam $\mathrm{t} \in[0, \mathrm{~T}]$ e $\mathrm{x}_{0} \in \mathrm{X}$. Se $\Gamma$ é convexo e limitado, entào $\mathcal{W}\left(\mathrm{t}, \mathrm{x}_{0}, \mathcal{U}_{\Gamma}\right)$ também é convexo e limitado.

Prova : Como $\mathcal{F}$ é linear, se $\mathrm{U} \subset \mathrm{G}([0, \mathrm{~T}], \mathrm{X})$ é convexo então também é convexo o conjunto $\mathcal{F} \mathbf{U}=\mathcal{W}(t, 0, \mathbf{U})$. Portanto.

$$
\mathcal{W}\left(\mathrm{t}, \mathrm{x}_{0}, \mathrm{U}\right)=\mathcal{F}(\mathrm{U})(\mathrm{t})+\mathrm{R}(\mathrm{t}, 0) \cdot \mathrm{x}_{0},
$$

é convexo. Quando $\mathrm{U}=\mathcal{U}_{\Gamma}$, segue da Proposição 2.2.4 que $\mathcal{U}_{\Gamma}$ é convexo. Portanto concluimos que $\mathcal{W}\left(\mathrm{t}, \mathrm{x}_{0}, \mathcal{U}_{\Gamma}\right)$ também é convexo. 
Como $\mathcal{F}$ é contínua, temos que $\|\mathcal{F}\|<\infty$. Além disso, $\mathrm{R} \in \mathrm{G}_{\mathrm{I}}^{\sigma} . \mathrm{SV} \mathrm{u}^{\mathrm{u}}$, isto é.

$$
\mathrm{SV}^{\mathrm{u}}[\mathrm{R}]=\sup _{0 \leq \mathrm{t} \leq \mathrm{T}} \mathrm{SV}\left[\mathrm{R}^{\mathrm{t}}\right]<\infty
$$

Assim, se U é limitado,

$$
\|\mathrm{y}\|=\left\|(\mathcal{F} \mathrm{u})(\mathrm{t})+\mathrm{R}(\mathrm{t}, 0) \cdot \mathrm{x}_{0}\right\| \leq\left\{1+\mathrm{SV}^{\mathrm{u}}[\mathrm{R}]\right\}\|\mathrm{u}\|+\left\|\mathrm{R}(\mathrm{t}, 0) \cdot \mathrm{x}_{0}\right\|<\infty,
$$

para todo $\mathrm{y} \in \mathcal{W}\left(\mathrm{t}, \mathrm{x}_{0}, \mathbf{U}\right)$. Em particular, vale a conclusão anterior quando $\mathrm{U}=\mathcal{U}_{\Gamma}$.

2.3.10 Proposição Sejam $t \in[0, \mathrm{~T}]$ e $\mathrm{x}_{0} \in \mathrm{X}$. Então valem as inclusōes

(a) $\mathcal{W}\left(\mathrm{t}, \mathrm{x}_{0}, \mathcal{U}_{\mathrm{r}}\right) \subset \mathcal{W}\left(\mathrm{t}, \mathrm{x}_{0}, \mathcal{U}\right)$.

(b) $\mathcal{W}\left(\mathrm{t}, \mathrm{x}_{0}, \lambda \mathcal{U}_{\Gamma}\right) \subset \mathcal{W}\left(\mathrm{t}, \mathrm{x}_{0}, \mathcal{U}_{\Gamma}\right)$, para cada $\lambda \in \mathbb{R}, 0 \leq \lambda \leq 1$

(c) $\mathcal{W}\left(\mathrm{t}, \mathrm{x}_{0}, \lambda \mathcal{U}_{\Gamma}\right) \subset \mathcal{W}\left(\mathrm{t}, \mathrm{x}_{0}, \eta \mathcal{U}_{\Gamma}\right)$, se $1<\lambda<\eta$.

(d) $\mathcal{W}\left(\mathrm{t}, \mathrm{x}_{0}, \mathcal{U}_{\lambda \mathrm{\Gamma}}\right) \subset \mathcal{W}\left(\mathrm{t}, \mathrm{x}_{0}, \mathcal{U}_{\Gamma}\right)$, para cada $\lambda \in \mathbb{R}, 0 \leq \lambda \leq 1$.

(e) $\mathcal{W}\left(\mathrm{t}, \mathrm{x}_{0}, \mathcal{U}_{\lambda \Gamma}\right) \subset \mathcal{W}\left(\mathrm{t}, \mathrm{x}_{0}, \mathcal{U}_{\eta \Gamma}\right)$, para $1 \leq \lambda<\eta$.

Prova : Sejam $U, V \subset G([0, T], X)$ tais que $U \subset V$. Então

$$
\mathcal{W}\left(\mathrm{t}, \mathrm{x}_{0}, \mathrm{U}\right) \subset \mathcal{W}\left(\mathrm{t}, \mathrm{x}_{0}, \mathrm{~V}\right)
$$

Realmente, se $\mathrm{z} \in \mathcal{W}\left(\mathrm{t}, \mathrm{x}_{0}, \mathrm{U}\right)$, entāo $\mathrm{z}=\mathrm{x}_{\mathrm{u}}(\mathrm{t})$, para algum $\mathrm{u} \in \mathrm{U}$. Como $\mathrm{u} \in \mathrm{V}$, segue que $\mathrm{z} \in \mathcal{W}\left(\mathrm{t}, \mathrm{x}_{0}, \mathbf{V}\right)$. Como $\lambda \mathbf{U} \subset \mathbf{U}$, para $0 \leq \lambda \leq 1$, e $\lambda \mathbf{U} \subset \eta \mathbf{U}$, para $1<\lambda<\eta$. temos que

$\mathcal{W}\left(\mathrm{t}, \mathrm{x}_{0}, \lambda \mathrm{U}\right) \subset \mathcal{W}\left(\mathrm{t}, \mathrm{x}_{0}, \mathrm{U}\right), 0 \leq \lambda<1$ 
$\mathcal{W}\left(\mathrm{t}, \mathrm{x}_{0}, \lambda \mathbf{U}\right) \subset \mathcal{W}\left(\mathrm{t}, \mathrm{x}_{0}, \eta \mathbf{U}\right)$, para $1<\lambda<\eta$

Em particular, quando $U=\mathcal{U}_{\Gamma}$, temos (a), (b) e (c). Da observação 2.2 .5 temos as afirmações (d) e (e).

2.3.11 Proposiçāo Seja $U \subset \mathrm{G}([0, \mathrm{~T}], \mathrm{X})$. Para quaisquer $\lambda \in \mathbb{R}, \mathrm{x}_{0} \in \mathrm{X}$ e $\mathrm{t} \in[0, \mathrm{~T}]$

$$
\mathcal{W}\left(\mathrm{t}, \mathrm{x}_{0}, \lambda \mathbf{U}\right)=\lambda \mathcal{W}\left(\mathrm{t}, \mathrm{x}_{0}, \mathbf{U}\right)+(1-\lambda) \mathrm{R}(\mathrm{t}, 0) \cdot \mathrm{x}_{0}
$$

Além disso, quando $\mathrm{x}_{0} \in \operatorname{Ker} \mathrm{R}(\mathrm{t}, 0)$,

$$
\mathcal{W}\left(\mathrm{t}, \mathrm{x}_{0}, \lambda \mathbf{U}\right)=\lambda \mathcal{W}\left(\mathrm{t}, \mathrm{x}_{0}, \mathbf{U}\right)
$$

Em particular, vale a fórmula anterior para $\mathbf{U}=\mathcal{U}_{\Gamma}$.

Prova : Como $\mathcal{F}$ é linear, $\mathcal{F}(\lambda U)=\lambda \mathcal{F}(\mathbf{U})$. Realmente, se $\mathrm{v} \in \lambda \boldsymbol{U}$, então $\mathrm{v}=\lambda \mathbf{u}$, para algum $u \in \mathbf{U}$. Logo,

$$
\left(\mathcal{F}_{\mathrm{v}}\right)(\mathrm{t})=(\mathcal{F})(\lambda \mathrm{u})(\mathrm{t})=\lambda(\mathcal{F} \mathrm{u})(\mathrm{t})
$$

Assim,

$$
\begin{gathered}
\mathcal{W}\left(\mathrm{t}, \mathrm{x}_{0}, \lambda \mathrm{U}\right)=\left\{\left(\mathcal{F}_{\mathrm{v}}\right)(\mathrm{t}) / \mathrm{v} \in \mathrm{U}+\mathrm{R}(\mathrm{t}, 0) \cdot \mathrm{x}_{0}=\lambda\{(\mathcal{F}) \mathrm{u}(\mathrm{t}) / \mathrm{u} \in \mathrm{U}\}+\right. \\
+\mathrm{R}(\mathrm{t}, 0) \cdot \mathrm{x}_{0}=\lambda\{(\mathcal{F}) \mathrm{u}(\mathrm{t}) / \mathrm{u} \in \mathrm{U}\}+\lambda \mathrm{R}(\mathrm{t}, 0) \cdot \mathrm{x}_{0}+(1-\lambda) \mathrm{R}(\mathrm{t} .0) \cdot \mathrm{x}_{0}=
\end{gathered}
$$




$$
=\lambda \mathcal{W}\left(\mathrm{t}, \mathrm{x}_{0}, \mathrm{U}\right)+(1-\lambda) \mathrm{R}(\mathrm{t}, 0) \cdot \mathrm{x}_{0} .
$$

2.3.12 Observação Quando $U$ é um subespaço de $G([0, T], X)$ e $x_{0} \in \operatorname{Ker} R(t, 0)$, o conjunto de atingibilidade $\mathcal{W}\left(\mathrm{t}, \mathrm{x}_{0}, \mathrm{U}\right)$ também é um subespaço de $\mathrm{X}$. Isto acontece, por exemplo, quando $\mathrm{U}=\mathcal{U}$.

2.3.13 Observação A Proposição 2.3 .11 garante que a aplicação

$$
\lambda \rightarrow \mathcal{W}\left(\mathrm{t}, \mathrm{x}_{0}, \lambda \mathcal{U}_{\Gamma}\right)
$$

é contínua em relação à métrica de Hausdorff. Além disso, a parte (c) da Proposição 2.3.10 garante sua monotonicidade (no sentido da inclusão).

2.3.14 Lema Seja $z \in X$. Então, para todo $t \in[0, T]$ : o controle

$$
\mathrm{u}(\mathrm{t})=\left[\mathrm{I}_{\mathrm{x}}-\mathrm{K}(\mathrm{t}, 0)\right] \cdot \mathrm{z}-\mathrm{x}_{0}
$$

é tal que $\mathrm{x}_{\mathrm{u}}(\mathrm{t})=(\mathcal{F} \mathrm{u})(\mathrm{t})+\mathrm{R}(\mathrm{t}, 0) \cdot \mathrm{x}_{0}=\mathrm{z}$.

Prova: De fato, se $u(t)=z-x_{0}-K(t, 0) \cdot z$,

$$
\int_{0}^{\mathrm{t}} \cdot \mathrm{d}_{\tau} \mathrm{R}(\mathrm{t}, \tau) \cdot\left[\mathrm{z}-\mathrm{x}_{0}-\mathrm{K}(\tau, 0) \cdot \mathrm{z}\right]=\int_{0}^{\mathrm{t}} \cdot \mathrm{d}_{\tau} \mathrm{R}(\mathrm{t}, \tau) \cdot\left[\mathrm{z}-\mathrm{x}_{0}\right]-\int_{0}^{\mathrm{t}} \cdot \mathrm{d}_{\tau} \mathrm{R}(\mathrm{t}, \tau) \circ \mathrm{K}(\tau, 0) \cdot \mathrm{z}=
$$




$$
\begin{gathered}
=[\mathrm{R}(\mathrm{t}, \mathrm{t})-\mathrm{R}(\mathrm{t}, 0)] \cdot\left(\mathrm{z}-\mathrm{x}_{0}\right)-\int_{0}^{\mathrm{t}} \cdot \mathrm{d}_{\tau} \mathrm{R}(\mathrm{t}, \tau) \circ \mathrm{K}(\tau, 0) \cdot \mathrm{z}=\left[\mathrm{I}_{\mathrm{x}}-\mathrm{R}(\mathrm{t}, 0)\right] \cdot\left(\mathrm{z}-\mathrm{x}_{0}\right) \\
-\int_{0}^{\mathrm{t}} \cdot \mathrm{d}_{\tau} \mathrm{R}(\mathrm{t}, \tau) \circ \mathrm{K}(\tau, 0) \cdot \mathrm{z}
\end{gathered}
$$

Como $R \in G_{I}^{\sigma} \cdot S V^{u}$, e portanto $R(t, t)=I_{x}$, é o resolvente de $K$, usando a fórmula $\left(R_{*}\right)$,

$$
\begin{gathered}
(\mathcal{F} \mathrm{u})(\mathrm{t})+\mathrm{R}(\mathrm{t}, 0) \cdot \mathrm{x}_{0}=\mathrm{z}-\mathrm{x}_{0}-\mathrm{K}(\mathrm{t}, 0) \cdot \mathrm{z}+\int_{0}^{\mathrm{t}} \cdot \mathrm{d}_{\tau} \mathrm{R}(\mathrm{t}, \tau) \cdot\left[\mathrm{z}-\mathrm{x}_{0}+\mathrm{K}(\tau, 0) \cdot \mathrm{z}\right]= \\
=\mathrm{z}-\mathrm{x}_{0}-\mathrm{K}(\mathrm{t}, 0) \cdot \mathrm{z}-\left[\mathrm{I}_{\mathrm{x}}-\mathrm{R}(\mathrm{t}, 0)\right] \cdot\left(\mathrm{z}-\mathrm{x}_{0}\right)+\int_{0}^{\mathrm{t}} \cdot \mathrm{d}_{\tau} \mathrm{R}(\mathrm{t}, \tau) \circ \mathrm{K}(\tau, 0) \cdot \mathrm{z}= \\
=\mathrm{R}(\mathrm{t}, 0) \cdot \mathrm{z}-\mathrm{K}(\mathrm{t}, 0) \cdot \mathrm{z}+\int_{0}^{\mathrm{t}} \cdot \mathrm{d}_{\tau} \mathrm{R}(\mathrm{t}, \tau) \circ \mathrm{K}(\tau, 0) \cdot \mathrm{z}=\mathrm{I}_{\mathrm{x}} \cdot \mathrm{z}=\mathrm{z} .
\end{gathered}
$$

2.3.15 Observação Da definição do conjunto de atingibilidade temos que, para cada $\mathrm{t} \in[0, \mathrm{~T}]$, se $\mathrm{z} \in \mathcal{W}\left(\mathrm{t}, \mathrm{x}_{0}, \mathcal{U}_{\Gamma}\right)$ então $\left[\mathrm{I}_{\mathrm{x}}-\mathrm{K}(\mathrm{t}, 0)\right] \cdot \mathrm{z}-\mathrm{x}_{0} \in \Gamma$. Realmente, se $\left[\mathrm{I}_{\mathrm{x}}-\mathrm{K}(\mathrm{t}, 0)\right] \cdot \mathrm{z}-\mathrm{x}_{0} \notin \Gamma$, para algum $\mathrm{t} \in[0, \mathrm{~T}]$, então $\mathrm{u} \notin \mathcal{U}_{\Gamma}$, e portanto, $(\mathcal{F} \mathrm{u})(\mathrm{t})+$ $\mathrm{R}(\mathrm{t}, 0) \cdot \mathrm{x}_{0}=\mathrm{z} \notin \mathcal{W}\left(\mathrm{t}, \mathrm{x}_{0}, \mathcal{U}_{\mathrm{r}}\right)$.

2.3.16 Proposição Para cada $\mathrm{t} \in[0, \mathrm{~T}]$ e $\mathrm{x}_{0} \in X$, se $\mathrm{U} \subset \mathrm{G}([0, \mathrm{~T}], \mathrm{X})$ é fechado, então $\mathcal{W}\left(\mathrm{t}, \mathrm{x}_{0}, \mathrm{U}\right)$ também é fechado.

Prova : Seja $\left(\mathrm{y}_{\mathrm{n}}\right)_{\mathrm{n} \in \mathbb{N}}$ uma sequência em $\mathcal{W}\left(\mathrm{t}, \mathrm{x}_{0}, \mathrm{U}\right)$ tal que $\mathrm{y}_{\mathrm{n}} \rightarrow \mathrm{y}$ em $\mathrm{X}$. Então, pelo lema anterior, a sequência $\left(u_{n}\right)_{n \in \mathbb{N}}$ dada por 


$$
\mathrm{u}_{\mathrm{n}}(\mathrm{t})=\mathrm{y}_{\mathrm{n}}-\mathrm{x}_{0}-\mathrm{K}(\mathrm{t}, 0) \cdot \mathrm{y}_{\mathrm{n}},
$$

é tal que $u_{n} \in \mathbf{U}$ e

$$
\mathrm{y}_{\mathrm{n}}=\left(\mathcal{F} \mathrm{u}_{\mathrm{n}}\right)(\mathrm{t})+\mathrm{R}(\mathrm{t}, 0) \cdot \mathrm{x}_{0}=\mathrm{u}_{\mathrm{n}}(\mathrm{t})+\mathrm{R}(\mathrm{t}, 0) \cdot \mathrm{x}_{0}-\int_{0}^{\mathrm{t}} \cdot \mathrm{d}_{\tau} \mathrm{R}(\mathrm{t}, \tau) \cdot \mathrm{u}_{\mathrm{n}}(\tau)
$$

Agora, para cada $\mathrm{t} \in[0, \mathrm{~T}]$ e $\mathrm{m}, \mathrm{n} \in \mathbb{N}$,

$$
\left\|\mathrm{u}_{\mathrm{n}}(\mathrm{t})-\mathrm{u}_{\mathrm{m}}(\mathrm{t})\right\|=\left\|\mathrm{y}_{\mathrm{n}}-\mathrm{y}_{\mathrm{m}}-\mathrm{K}(\mathrm{t}, 0) \cdot\left(\mathrm{y}_{\mathrm{n}}-\mathrm{y}_{\mathrm{m}}\right)\right\| \leq\left(1+S V^{\mathrm{u}}[K]\right)\left\|\mathrm{y}_{\mathrm{n}}-\mathrm{y}_{\mathrm{m}}\right\|
$$

Portanto,

$$
\left\|\mathrm{u}_{\mathrm{n}}-\mathrm{u}_{\mathrm{m}}\right\| \leq\left(1+S \mathrm{~V}^{\mathrm{u}}[\mathrm{K}]\right)\left\|\mathrm{y}_{\mathrm{n}}-\mathrm{y}_{\mathrm{m}}\right\|
$$

Como $\left(\mathrm{y}_{\mathrm{n}}\right)_{\mathrm{n} \in \mathbb{N}}$ é uma sequência de Cauchy em $\mathrm{X}$ e $\mathrm{K} \in \mathrm{G}_{0}^{\sigma} \cdot \mathrm{SV}^{\mathrm{u}}$, segue da desigualdade anterior que $\left(u_{n}\right)_{n \in \mathbb{N}}$ é uma sequência de Cauchy em $U \subset G([0, T], X)$, isto é. existe $\mathrm{u} \in \mathrm{G}([0, \mathrm{~T}] . \mathrm{X})$ tal que $\mathrm{u}_{\mathrm{n}} \rightarrow \mathrm{u}$ uniformente. Como $\mathrm{U}$ é fechado segue que $\mathrm{u} \in \mathbf{U}$. Da continuidade de $\mathcal{F}$ temos que, $\left(\mathcal{F} \mathbf{u}_{n}\right) \rightarrow(\mathcal{F} u)$ uniformemente. Logo,

$$
\mathrm{y}_{\mathrm{n}}=\left(\mathcal{F} \mathrm{u}_{\mathrm{n}}\right)(\mathrm{t})+\mathrm{R}(\mathrm{t}, 0) \cdot \mathrm{x}_{0} \rightarrow(\mathcal{F} \mathrm{u})(\mathrm{t}) \mathrm{em} \mathrm{X}
$$

para todo $t \in[0, T]$. Segue portanto, da unicidade do limite, que

$$
\mathrm{y}=(\mathcal{F} \mathrm{u})(\mathrm{t}), \operatorname{com} \mathrm{u} \in \mathbf{U} \text {. isto é, } \mathrm{y} \in \mathcal{W}\left(\mathrm{t}, \mathrm{x}_{0} . \mathrm{U}\right)
$$


Portanto $\mathcal{W}\left(\mathrm{t}, \mathrm{x}_{0}, \mathrm{U}\right)$ é fechado.

2.3.17 Proposição Para cada $t \in[0, T]$ e $x_{0} \in X$, suponhamos que $\Gamma \subset X$ é fechado. Então $\mathcal{W}\left(\mathrm{t}, \mathrm{x}_{0}, \mathcal{U}_{\mathrm{r}}\right)$ também é fechado.

Prova : Segue da Proposição anterior e da Proposição 2.2.4.

2.3.18 Proposição Sejam $\mathrm{t} \in[0, \mathrm{~T}]$ e $\mathrm{x}_{0} \in \mathrm{X}$. Então int $\mathcal{W}\left(\mathrm{t}, \mathrm{x}_{0}, \mathcal{U}_{\Gamma}\right)=\varnothing$, para qualquer $\Gamma \subset \mathrm{X}$.

Prova : Sejam $U \subset \mathrm{G}([0, \mathrm{~T}], \mathrm{X}), \mathrm{r}>0$ e consideremos $\mathrm{z} \in \mathcal{W}\left(\mathrm{t}, \mathrm{x}_{0}, \mathrm{U}\right)$. Pelo Lema 2.3.14, o controle $u(t)=\left[I_{x}-K(t, 0)\right] z-x_{0}$ é tal que $u \in U$ e

$$
\mathrm{z}=(\mathcal{F} \mathrm{u})(\mathrm{t})+\mathrm{R}(\mathrm{t}, 0) \cdot \mathrm{x}_{0}
$$

Como int $\mathbf{U}=\emptyset$, para $\overline{\mathrm{r}}=\frac{\mathrm{r}}{\|\mathcal{F}\|}>0$ existe $\mathrm{v} \in \mathbf{U}$ tal que

$$
\mathrm{v} \in \mathrm{B}_{\overline{\mathrm{r}}}(\mathrm{u}) \text { e } \mathrm{v} \notin \mathrm{U} \text {. }
$$

Consideremos $\mathrm{y}=\left(\mathcal{F}_{\mathrm{v}}\right)(\mathrm{t})+\mathrm{R}(\mathrm{t}, 0) \cdot \mathrm{x}_{0}$. Então, como $\|\mathrm{v}-\mathrm{u}\|<\overline{\mathrm{r}}$,

$$
\|\mathrm{y}-\mathrm{z}\|=\|\mathcal{F}(\mathrm{v}-\mathrm{u})(\mathrm{t})\| \leq\|\mathcal{F}\|\|\mathrm{v}-\mathrm{u}\|<\mathrm{r} .
$$

Portanto $\mathrm{y} \in \mathrm{B}_{\mathrm{r}}(\mathrm{z})$. No entanto, como $\mathrm{v} \notin \mathrm{U}$, temos que $\mathrm{y} \notin \mathcal{W}\left(\mathrm{t}, \mathrm{x}_{0}\right.$. U $)$. Em particular. como int $\mathcal{U}_{\Gamma}=\varnothing$. temos o resultado. 
2.3.19 Observação Lembrando que $\mathcal{U}_{\Gamma}$ está contido no subespaço próprio $\mathcal{U}$ de $\mathrm{G}([0, \mathrm{~T}], \mathrm{X})$, e da Observação 2.3.2 (b), segue que $\mathcal{W}\left(\mathrm{t}, \mathrm{x}_{0}, \mathcal{U}_{\Gamma}\right)$ está contido na variedade linear $\mathcal{W}\left(\mathrm{t}, \mathrm{x}_{0}, \mathcal{U}\right)=\mathcal{W}(\mathrm{t}, 0, \mathcal{U})+\mathrm{R}(\mathrm{t}, 0) \cdot \mathrm{x}_{0}$ e que $\mathcal{W}\left(\mathrm{t}, 0, \mathcal{U}_{\mathrm{r}}\right)$ está contido no subespaço $\mathcal{W}(\mathrm{t}, 0, \mathcal{U})$.

Se considerarmos o conjunto restrição $\Gamma$ convexo teremos $\mathcal{U}_{\Gamma}$ convexo, e portanto $\mathcal{W}\left(\mathrm{t}, \mathrm{x}_{0}, \mathcal{U}_{\Gamma}\right)$ também convexo. Portanto tem sentido considerar os elementos extremos destes três conjuntos.

2.3.20 Definição Dizemos que um controle u $\in \mathcal{U}_{\Gamma}$ é $\Gamma$-extremal no intervalo $[0, t]$, para $t \in[0, T]$, se a resposta correspondente $\mathrm{x}_{\mathrm{u}}$, dada pela fórmula $(\rho)$, é tal que

$$
\mathrm{x}_{\mathrm{u}}(\mathrm{t}) \in \partial_{\mathrm{rel}} \mathcal{W}\left(\mathrm{t}, \mathrm{x}_{0}, \mathcal{U}_{\mathbf{r}}\right)
$$

2.3.21 Proposição Suponhamos que u $\in \partial \mathcal{U}_{\Gamma}$. Então u é um controle $\Gamma$-extremal. Em outras palavras,

$$
\mathcal{W}\left(\mathrm{t}, \mathrm{x}_{0}, \partial \mathcal{U}_{\Gamma}\right) \subset \partial_{\text {rel }} \mathcal{W}\left(\mathrm{t}, \mathrm{x}_{0}, \mathcal{U}_{\Gamma}\right)
$$

Prova : Sejam $\mathbf{U} \subset \mathrm{G}([0, \mathrm{~T}], \mathrm{X}), \mathrm{z} \in \mathcal{W}\left(\mathrm{t}, \mathrm{x}_{0}, \partial \mathbf{U}\right)$ e $\mathrm{r}>0$. Então existe $\mathrm{u} \in \partial \mathrm{U}$ tal que

$$
\mathrm{z}=\mathrm{x}_{\mathrm{u}}(\mathrm{t})=(\mathcal{F} \mathrm{u})(\mathrm{t})+\mathrm{R}(\mathrm{t}, 0) \cdot \mathrm{x}_{0}
$$

Como $\mathrm{u} \in \partial \mathbf{U}$ : dado $\overline{\mathrm{r}}=\frac{\mathrm{r}}{\|\mathcal{F}\|}>0$, existem $\mathrm{v}_{1} \cdot \mathrm{v}_{2} \in \mathrm{G}([0, \mathrm{~T}] . \mathrm{X})$ tais que $\mathrm{v}_{1} \cdot \mathrm{v}_{2} \in \mathrm{B}_{\mathrm{r}}(\mathrm{u})$. $\mathrm{v}_{1} \in \mathrm{U}$ e $\mathrm{v}_{2} \in \mathrm{G}([0, \mathrm{~T}], \mathrm{X}) \backslash \mathrm{U}$. Consideremos, para $\mathrm{i}=1,2$, 


$$
\mathrm{y}_{\mathbf{i}}=\left(\mathcal{F}_{\mathbf{v}_{\mathrm{i}}}\right)(\mathrm{t})+\mathrm{R}(\mathrm{t}, 0) \cdot \mathrm{x}_{0}
$$

Então $\mathrm{y}_{1} \in \mathcal{W}\left(\mathrm{t}, \mathrm{x}_{0}, \mathrm{U}\right)$ e $\mathrm{y}_{2} \in \mathrm{X} \backslash \mathcal{W}\left(\mathrm{t}, \mathrm{x}_{0}, \mathbf{U}\right)$. Além disso, para $\mathrm{i}=1,2$,

$$
\left\|\mathrm{y}_{\mathrm{i}}-\mathrm{z}\right\|=\left\|\left(\mathcal{F}_{\mathrm{v}_{\mathrm{i}}}\right)(\mathrm{t})-(\mathcal{F} \mathrm{u})(\mathrm{t})\right\| \leq\left\|\mathcal{F}\left(\mathrm{v}_{\mathrm{i}}-\mathrm{u}\right)\right\| \leq\|\mathcal{F}\|\left\|\mathrm{v}_{\mathrm{i}}-\mathrm{u}\right\|<\mathrm{r},
$$

isto é, $\mathrm{y}_{\mathrm{i}} \in \mathrm{B}_{\mathrm{r}}(\mathrm{z})$. Portanto, $\mathrm{z} \in \partial \mathcal{W}\left(\mathrm{t}, \mathrm{x}_{0}, \mathbf{U}\right)$. Em particular, para $\mathbf{U}=\mathcal{U}_{\mathbf{r}}$, temos o resultado.

Dados $\mathrm{W} \subset \mathrm{X}$ e $\mathrm{V} \subset \mathrm{X}^{\prime}$, sejam

$$
\begin{aligned}
& \mathrm{W}^{\mathrm{o}}=\left\{\mathrm{x}^{\prime} \in \mathrm{X}^{\prime} / \mathrm{x}^{\prime}(\mathrm{x})=0, \text { para todo } \mathrm{x} \in \mathrm{W}\right\} \\
& { }^{\circ} \mathrm{V}=\left\{\mathrm{x} \in \mathrm{X} / \mathrm{x}^{\prime}(\mathrm{x})=0, \text { para todo } \mathrm{x}^{\prime} \in \mathrm{V}\right\}
\end{aligned}
$$

os conjuntos anuladores de $\mathrm{W}$ e $\mathrm{V}$, respectivamente. Se $\mathrm{W}$ é um subconjunto de $\mathrm{X}$ e $[W]$ é o subespaço fechado gerado por $W$, então $[W]={ }^{\circ}\left(W^{\circ}\right)($ ver $[15]$, Lemma 3.3, p. 61 ). Para cada $\mathrm{t} \in[0, \mathrm{~T}]$, vamos denotar por

$$
\mathrm{S}(\mathrm{t})={ }^{\circ}\left(\mathcal{W}\left(\mathrm{t}, 0, \mathcal{U}_{\Gamma}\right)^{\circ}\right)
$$

e vamos considerar, respectivamente, a fronteira e o interior de $\mathcal{W}\left(\mathrm{t}, 0, \mathcal{U}_{\Gamma}\right)$ relativos a $\mathrm{S}(\mathrm{t})$

$$
\operatorname{int}_{\mathrm{S}(\mathrm{t})} \mathcal{W}\left(\mathrm{t} .0 . \mathcal{U}_{\mathrm{r}}\right)=\mathrm{s}(\mathrm{t}) \cap \operatorname{int} \mathcal{W}\left(\mathrm{t}, 0 . \mathcal{U}_{\mathrm{r}}\right)
$$




$$
\partial_{\mathrm{S}(\mathrm{t})} \mathcal{W}\left(\mathrm{t}, 0, \mathcal{U}_{\mathrm{r}}\right)=\mathrm{S}(\mathrm{t}) \cap \partial \mathcal{W}\left(\mathrm{t}, 0, \mathcal{U}_{\Gamma}\right)
$$

2.3.22 Proposição Suponhamos que int $\Gamma \neq \emptyset$. Então, para cada $t \in[0, T]$,

$$
\mathrm{s}(\mathrm{t})=\mathcal{W}(\mathrm{t}, 0, \mathcal{U})
$$

Prova : Pela parte (a) da proposição 2.3.10, $\mathcal{W}\left(\mathrm{t}, 0, \mathcal{U}_{\Gamma}\right) \subset \mathcal{W}(\mathrm{t}, 0, \mathcal{U})$. Além disso, pela proposição $2.3 .17, \mathcal{W}(\mathrm{t}, 0, \mathcal{U})$ é fechado, e portanto,

$$
\left[\mathcal{W}\left(\mathrm{t}, 0, \mathcal{U}_{\mathrm{r}}\right)\right] \subset \mathcal{W}(\mathrm{t}, 0, \mathcal{U})
$$

Por outro lado, se $\mathrm{z} \in \mathcal{W}(\mathrm{t}, 0, \mathcal{U})$, então $\mathrm{z}=(\mathcal{F} \mathrm{u})(\mathrm{t})$, para algum $\mathrm{u} \in \mathcal{U}$. Como $\mathcal{U}=\left[\mathcal{U}_{\Gamma}\right]$ ( ver proposição 2.2 .10 ),

$$
\mathrm{u}=\sum_{\mathrm{j}=1}^{\mathrm{n}} \alpha_{\mathrm{j}} \mathrm{u}_{\mathrm{j}}
$$

$\mathrm{u}_{\mathrm{j}} \in \mathcal{U}_{\Gamma}$ e para escalares $\alpha_{\mathrm{j}}$

Portanto,

$$
\mathrm{z}=(\mathcal{F} \mathrm{u})(\mathrm{t})=\left(\mathcal{F} \sum_{\mathrm{j}=1}^{\mathrm{n}} \alpha_{\mathrm{j}} \mathrm{u}_{\mathrm{j}}\right)(\mathrm{t})=\sum_{\mathrm{j}=1}^{\mathrm{n}} \alpha_{\mathrm{j}}\left(\mathcal{F} \mathrm{u}_{\mathrm{j}}\right)(\mathrm{t})
$$

Como $\mathrm{u}_{\mathrm{j}} \in \mathcal{W}\left(\mathrm{t}, 0, \mathcal{U}_{\Gamma}\right)$, para $\mathrm{j}=1 \ldots, \mathrm{n}$, segue que $\mathrm{z} \in\left[\mathcal{W}\left(\mathrm{t}, 0, \mathcal{U}_{\mathrm{r}}\right)\right]$. Logo,

$$
\mathcal{W}(\mathrm{t}, 0 . \mathcal{U}) \subset\left[\mathcal{W}\left(\mathrm{t} .0 . \mathcal{U}_{\Gamma}\right)\right]
$$


2.3.23 Proposição Suponhamos que int $\Gamma \neq \emptyset$. Então, para cada $t \in[0, T]$ :

$$
\operatorname{int}_{\mathrm{S}(\mathrm{t})} \mathcal{W}\left(\mathrm{t}, 0, \mathcal{U}_{\mathrm{r}}\right) \neq \varnothing
$$

Prova : Consideremos a aplicação avaliação no instante t,

$$
\mathcal{F}_{\mathrm{t}}: \mathrm{u} \in \mathrm{G}([0, \mathrm{~T}], \mathrm{X}) \longmapsto(\mathcal{F} \mathrm{u})(\mathrm{t}) \in \mathrm{X}
$$

Então, $\mathcal{F}_{\mathrm{t}}$ é linear e

. $\mathcal{F}_{\mathrm{t}}$ é contínua, pois se $\mathrm{u}, \mathrm{v} \in \mathrm{G}([0, \mathrm{~T}], \mathrm{X})$,

$$
\left\|\mathcal{F}_{\mathrm{t}} \mathrm{u}-\mathcal{F}_{\mathrm{t}} \mathrm{v}\right\|=\|\mathrm{u}(\mathrm{t})-\mathrm{v}(\mathrm{t})\| \leq\|\mathrm{u}-\mathrm{v}\|
$$

. $\mathcal{F}_{\mathrm{t}}$ é sobrejetora, já que se $\mathrm{y} \in \mathrm{X}$, a função $\mathrm{u}(\mathrm{t})=\left[\mathrm{I}_{\mathrm{x}}-\mathrm{K}(\mathrm{t}, 0)\right] \cdot \mathrm{y}, \mathrm{t} \in[0, \mathrm{~T}]$, é regrada e $\mathcal{F}_{\mathrm{t}} \mathrm{u}=\mathrm{y}$.

Portanto, pelo Teorema da Aplicação Aberta, $\mathcal{F}_{\mathrm{t}}$ é uma aplicação aberta. Como, pela proposição 2.2.4, int $_{\text {rel }} \mathcal{U}_{\Gamma} \neq \varnothing$, segue que $\mathcal{F}_{\mathrm{t}}$ (int rel $_{\mathcal{U}_{\mathrm{\Gamma}}}$ ) é um subconjunto aberto e não-vazio. Pela parte (a) da proposição 2.3.5,

$$
\mathcal{F}_{\mathrm{t}}\left(\text { int }_{\text {rel }} \mathcal{U}_{\Gamma}\right)=\mathcal{W}\left(\mathrm{t}, 0, \text { int }_{\text {rel }} \mathcal{U}_{\Gamma}\right) \subset \mathcal{W}\left(\mathrm{t}, 0, \mathcal{U}_{\Gamma}\right) \subset \mathcal{W}(\mathrm{t} .0 . \mathcal{U})=\mathrm{S}(\mathrm{t})
$$

e portanto, $\operatorname{int}_{\mathrm{S}(\mathrm{t})} \mathcal{W}\left(\mathrm{t}, 0, \mathcal{U}_{\Gamma}\right) \neq \emptyset$.

2.3.24 Proposição $\operatorname{Se} u \in \partial_{\mathrm{r}} \mathcal{U}_{\mathrm{\Gamma}}$, então, para cada $t \in[0, T]$,

$$
(\mathcal{F} \mathbf{u})(\mathrm{t}) \in \partial_{\mathrm{S}(\mathrm{t})} \mathcal{W}\left(\mathrm{t} .0 . \mathcal{U}_{\Gamma}\right)
$$


Prova : Seja $\mathrm{y}=(\mathcal{F} \mathrm{u})(\mathrm{t})$, para cada $\mathrm{t} \in[0, \mathrm{~T}]$, e consideremos $\mathrm{B}_{\mathrm{r}}(\mathrm{y}), \mathrm{r}>0$. Se $\mathrm{u} \in \partial_{\mathrm{r}} \mathcal{U}_{\Gamma}$. então, para $\overline{\mathrm{r}}=\frac{\mathrm{r}}{\|\mathcal{F}\|}>0$, existem $\mathrm{v}_{1}, \mathrm{v}_{2} \in \mathrm{B}_{\overline{\mathrm{r}}}(\mathrm{u}) \subset \mathcal{U}$ tais que $\mathrm{v}_{1} \in \mathcal{U}_{\mathrm{r}}$ e $\mathrm{v}_{2} \notin \mathcal{U}_{\mathrm{r}}$. Desse modo, $\mathrm{z}_{1}=\left(\mathcal{F}_{\mathrm{v}_{1}}\right)(\mathrm{t}) \in \mathcal{W}\left(\mathrm{t}, 0, \mathcal{U}_{\Gamma}\right)$ e $\mathrm{z}_{2}=\left(\mathcal{F}_{\mathrm{v}_{2}}\right)(\mathrm{t}) \notin \mathcal{W}\left(\mathrm{t}, 0, \mathcal{U}_{\Gamma}\right)$. Além disso, para $\mathrm{i}=1,2$,

$$
\left\|\mathrm{z}_{\mathrm{i}}-\mathrm{y}\right\|=\left\|\left(\mathcal{F}_{\mathrm{v}_{\mathrm{i}}}\right)(\mathrm{t})-(\mathcal{F} \mathrm{u})(\mathrm{t})\right\| \leq \|\left(\mathcal{F}\|\| \mathrm{v}_{\mathrm{i}}-\mathrm{u} \|<\mathrm{r}\right.
$$

e portanto, $\mathrm{z}_{\mathrm{i}} \in \mathrm{B}_{\mathrm{r}}(\mathrm{y})$. Logo $\mathrm{y} \in \partial_{\mathrm{S}(\mathrm{t})} \mathcal{W}\left(\mathrm{t}, 0, \mathcal{U}_{\Gamma}\right)$.

2.3.25 Observação A proposição anterior, junto com a proposição 2.2.6, garante a existência de controles $\Gamma$-extremais constantes. Em outras palavras, se $\gamma \in \partial \Gamma$, então o controle constante $\dot{u}_{\gamma}(\mathrm{t})=\gamma, \mathrm{t} \in[0, \mathrm{~T}]$ é tal que

$$
\left(\mathcal{F} \mathrm{u}_{\gamma}\right)(\mathrm{t}) \in \partial_{\mathrm{s}(\mathrm{t})} \mathcal{W}\left(\mathrm{t}, 0, \mathcal{U}_{\Gamma}\right)
$$

Como $\left(\mathcal{F} \mathrm{u}_{\gamma}\right)(\mathrm{t})=\mathrm{R}(\mathrm{t}, 0) \cdot \gamma$, temos que,

$$
\text { se } \gamma \in \partial \Gamma \text {, então } \mathrm{R}(\mathrm{t}, 0) \cdot \gamma \in \partial_{\mathrm{S}(\mathrm{t})} \mathcal{W}\left(\mathrm{t}, 0, \mathcal{U}_{\Gamma}\right)
$$

2.3.26 Observação As aplicações $\mathcal{F}_{\mathrm{t}}$ em (2.41) também serão usadas no próximo capítulo para definirmos os funcionais custo. 


\section{Capítulo 3}

\section{Existência de Controles Ótimos :}

\section{Um Princípio do Máximo}

\subsection{Introdução}

Neste capítulo, que é o núcleo do trabalho, pretendemos mostrar alguns resultados que garantam a existência e unicidade de controles ótimos, referentes aos custos funcionais que serão definidos a seguir, e estabelecer um Princípio do Máximo que vai caracterizar todo controle ótimo como um controle extremal. Tal Princípio do Máximo suporta, em última instância, a formulação de um algoritmo que permite determinar um controle ótimo.

O problema de controle que vamos considerar a seguir tem quatro pressupostos básicos.

$\left(\mathrm{H}_{1}\right) \mathrm{O}$ núcleo $\mathrm{K} \in \mathrm{G}_{0}^{\sigma} \cdot \mathrm{SV}^{\mathrm{u}}$ tem um único resolvente $\mathrm{R} \in \mathrm{G}_{\mathrm{I}}^{\sigma} \cdot \mathrm{SV}^{\mathrm{u}}$.

$\left(\mathrm{H}_{2}\right) \mathrm{J}(0) \mathrm{X}$ é um subespaço vetorial fechado de $\mathrm{X}$ ( v. Teor 1.5.2). 
$\left(\mathrm{H}_{3}\right) \Gamma \subset \mathrm{X}$ é um subconjunto não-vazio, convexo, fechado. limitado e com interior nàovazio.

$\left(\mathrm{H}_{4}\right) \alpha \in \mathrm{SV}_{0}([0, \mathrm{~T}], \mathrm{L}(\mathrm{X}))$.

Consideremos, sob as hipóteses anteriores, o seguinte problema de controle ótimo, que denotaremos por $(\mathcal{P})_{0, \mathrm{~T}, \mathrm{x}_{0}}$ :

Maximizar um funcional $\mathcal{L}: \mathrm{G}([0, \mathrm{~T}], \mathrm{X}) \rightarrow \mathbb{R}, \quad$ sujeito à :

dinâmica :

$$
\mathrm{x}(\mathrm{t})-\mathrm{x}_{\mathrm{o}}+\int_{0}^{\mathrm{t}} \cdot \mathrm{d}_{\mathrm{s}} \mathrm{K}(\mathrm{t}, \mathrm{s}) \cdot \mathrm{x}(\mathrm{s})=\mathrm{u}(\mathrm{t}), 0 \leq \mathrm{t} \leq \mathrm{T}<\infty
$$

restrição linear :

$$
\mathrm{F}_{\alpha}[\mathrm{x}]=\int_{0}^{\mathrm{T}} \cdot \mathrm{d} \alpha(\mathrm{s}) \cdot \mathrm{x}(\mathrm{s})=0
$$

restrição sobre os controles :

$$
\mathrm{u} \in \mathcal{U}_{\Gamma}
$$

Lembramos que $\mathrm{X}$ é um espaço de Banach, $\mathrm{x}_{0} \in \mathrm{X}$ e $\mathrm{x}, \mathrm{u} \in \mathrm{G}([0, \mathrm{~T}] . \mathrm{X})$. A restrição nos controles $(\Gamma)$, aliada às hipóteses $\left(\mathrm{H}_{\mathrm{i}}^{\prime} \mathrm{s}\right)$ garantem, via teorema 1.5 .2 , que cada força 
$\mathrm{u} \in \mathcal{U}_{\Gamma}$ produz uma solução $\mathrm{x}_{\mathrm{u}} \in \mathrm{G}([0, \mathrm{~T}], \mathrm{X})$ do sistema $(\mathrm{K})+\left(\mathrm{F}_{\alpha}\right)_{0}$. Em outras palavras. $\mathcal{U}_{\Gamma}$ é o subconjunto das funções regradas u $:[0, \mathrm{~T}] \rightarrow \mathrm{X}$ que são eficazes no sentido de que produzem soluções da equação $(\mathrm{K})$ que realizam a condição $\left(\mathrm{F}_{\alpha}\right)_{0}$.

Para cada instante $\overline{\mathrm{t}} \in[0, \mathrm{~T}]$, vamos considerar neste capítulo, funcionais custo $\mathcal{L}(\overline{\mathrm{t}}): \mathrm{G}([0, \mathrm{~T}], \mathrm{X}) \rightarrow \mathbb{R}$, nas formas $:$

$$
\begin{gathered}
\mathcal{L}(\overline{\mathrm{t}}) \mathrm{u}=\left\langle\varphi, \mathrm{x}_{\mathrm{u}}(\overline{\mathrm{t}})>,\right. \\
\mathcal{L}(0, \overline{\mathrm{t}}) \mathrm{u}=\int_{0}^{\overline{\mathrm{t}}} \cdot\left\langle\mathrm{d} \gamma(\mathrm{s}), \mathrm{x}_{\mathrm{u}}(\mathrm{s})>\right.
\end{gathered}
$$

sendo que as funções $\varphi, \gamma$ serão definidas convenientemente nos parágrafos 3.2, 3.4. Além disso, no parágrafo 3.5 faremos algumas observações sobre programação dinâmica, cuja abordagem envolve o estudo de funcionais do tipo

$$
\mathcal{L}(\overline{\mathrm{t}}, \mathrm{T})=\left\langle\varphi, \mathrm{x}_{\mathrm{u}}(\mathrm{T})\right\rangle+\int_{\overline{\mathrm{t}}}^{\mathrm{T}} \cdot\left\langle\mathrm{d} \gamma(\mathrm{s}), \mathrm{x}_{\mathrm{u}}(\mathrm{s})\right\rangle
$$

\subsection{Funcional Avaliação no Instante $\overline{\mathbf{t}}$}

Consideremos

$$
\varphi \in \mathrm{S}(\overline{\mathrm{t}})^{\prime}, \hat{\varphi} \neq 0
$$

isto é, f é um funcional linear contínuo não-nulo definido no subespaço fechado $\mathcal{W}(\overline{\mathrm{t}}, 0, \mathcal{U})$ gerado por $\mathcal{W}\left(\overline{\mathrm{t}}, 0, \mathcal{U}_{\Gamma}\right)$. Sob as hipóteses $\left(\mathrm{H}_{\mathrm{i}}\right), \mathrm{i}=1, \ldots, 4$, para cada $\overline{\mathrm{t}} \in[0, \mathrm{~T}]$, vamos considerar, em primeiro lugar os funcionais custo $\mathcal{L}(\overline{\mathrm{t}}): \mathrm{G}([0, \mathrm{~T}], \mathrm{X}) \rightarrow \mathbb{R}$ da forma 


$$
\mathcal{L}(\overline{\mathrm{t}}) \mathrm{u}=\left\langle\varphi, \mathrm{x}_{\mathrm{u}}(\overline{\mathrm{t}})>\right.
$$

sendo $\mathrm{x}_{\mathrm{u}}$ a solução de $(\mathrm{K})+\left(\mathrm{F}_{\alpha}\right)_{0}+(\Gamma)$ associada a cada controle $\mathrm{u} \in \mathcal{U}_{\mathrm{\Gamma}}$. Dessa maneira, $\mathcal{L}(\overline{\mathrm{t}})$ não é um funcional linear ( isto ocorre se, e somente se, $\mathrm{x}_{0} \in \operatorname{Ker} \mathrm{R}(\overline{\mathrm{t}}, 0)$ ) porém

$$
\mathcal{L}(\overline{\mathrm{t}}) \mathrm{u}=\left\langle\varphi,(\mathcal{F} \mathrm{u})(\overline{\mathrm{t}})>+<\varphi \circ \mathrm{R}(\overline{\mathrm{t}}, 0), \mathrm{x}_{0}>\right.
$$

Para cada $c \in \mathbb{R}$, denotamos os hiperplanos de nível c de $\mathcal{L}(\bar{t})$ e de $\varphi$, respectivamente em $G([0, T], X), S(\bar{t})$, e em $S(\bar{t})+R(\bar{t}, 0) \cdot x_{0}$, por

$$
\begin{gathered}
\mathcal{A}(\varphi, \mathrm{c})=\left\{\mathrm{u} \in \mathrm{G}([0, \mathrm{~T}], \mathrm{X}) / \mathcal{L}(\overline{\mathrm{t}}) \mathrm{u}=<\varphi, \mathrm{x}_{\mathrm{u}}(\overline{\mathrm{t}})>=\mathrm{c}\right\} \cap \mathcal{U}, \\
\mathcal{H}_{\varphi}(\mathrm{c})=\{\mathrm{x} \in \mathrm{X} /<\varphi, \mathrm{x}>=\mathrm{c}\}, \quad \text { e } \quad \mathcal{K}_{\varphi}(\mathrm{c})=\mathcal{H}_{\varphi}(\mathrm{c})+\mathrm{R}(\overline{\mathrm{t}}, 0) \cdot \mathrm{x}_{0}
\end{gathered}
$$

3.2.1 Observação Com as notações anteriores é claro que $\mathrm{u} \in \mathcal{A}(\varphi, \mathrm{c}) \cap \mathcal{U}_{\Gamma}$, se, e somente se, $\mathrm{x}_{\mathrm{u}}(\overline{\mathrm{t}}) \in \mathcal{K}_{\varphi}(\mathrm{c}) \cap \mathcal{W}\left(\overline{\mathrm{t}}, \mathrm{x}_{0}, \mathcal{U}_{\Gamma}\right)$, se, e somente se, $(\mathcal{F} \mathrm{u})(\overline{\mathrm{t}}) \in \mathcal{H}_{\varphi}(\mathrm{c}) \cap \mathcal{W}\left(\overline{\mathrm{t}}, 0, \mathcal{U}_{\Gamma}\right)$. Portanto, $\mathcal{A}(\varphi, \mathrm{c}) \cap \mathcal{U}_{\Gamma} \neq \emptyset$, se, e somente se, $\mathcal{K}_{\varphi}(\mathrm{c}) \cap \mathcal{W}\left(\overline{\mathrm{t}}, \mathrm{x}_{0}, \mathcal{U}_{\Gamma}\right) \neq \emptyset$, se, e somente se, $\mathcal{H}_{\varphi}(\mathrm{c}) \cap \mathcal{W}\left(\overline{\mathrm{t}}, 0, \mathcal{U}_{\Gamma}\right) \neq \varnothing$.

3.2.2 Observação Como $\mathcal{W}\left(\overline{\mathrm{t}}, \mathrm{x}_{0}, \mathcal{U}_{\Gamma}\right) \neq \varnothing$, já que $\Gamma \neq \varnothing$, a condição $\varphi \neq 0$ implica que, para algum controle $\mathrm{u} \in \mathcal{U}_{\Gamma}, \mathcal{L}(\overline{\mathrm{t}}) \mathrm{u}=<\varphi, \mathrm{x}_{\mathrm{u}}(\overline{\mathrm{t}})>\neq 0$, e portanto, $\left.\mathcal{L}(\overline{\mathrm{t}})\right|_{\mathcal{u}_{\Gamma}} \neq 0$. Logo 
que, para algum controle $\mathrm{u} \in \mathcal{U}_{\Gamma}, \mathcal{L}(\overline{\mathrm{t}}) \mathrm{u}=<\varphi, \mathrm{x}_{\mathrm{u}}(\overline{\mathrm{t}})>\neq 0$, e portanto, $\left.\mathcal{L}(\overline{\mathrm{t}})\right|_{u_{\Gamma}} \neq 0$. Logo $\left.\mathcal{L}\left(\overline{\mathrm{t}}_{\mathrm{.}}\right)\right|_{u} \neq 0$. Assim, se existir um $c_{0} \in \mathbb{R}$ tal que $\mathcal{A}\left(\varphi, c_{0}\right)=\mathcal{U}_{\Gamma}$, então $\mathcal{L}\left(\overline{\mathrm{t}}_{\mathrm{t}}\right) \mathrm{u}=\mathrm{c}_{0}$, para todo $\mathrm{u} \in \mathcal{U}_{\Gamma}$. Portanto,

$$
\mathcal{A}(\varphi, \mathrm{c}) \neq \mathcal{U}, \text { para todo c } \in \mathbb{R}
$$

isto é, $\mathcal{A}(\varphi, \mathrm{c})$ e $\mathcal{U}$ são "transversais ". Na verdade a condição $\varphi \neq 0, \varphi \in \mathrm{S}(\overline{\mathrm{t}})^{\prime}$, é equivalente à condição $\varphi \in \mathrm{X}^{\prime}$ tal que $\mathcal{W}\left(\overline{\mathrm{t}}, \mathrm{x}_{0}, \mathcal{U}\right) \neq\{\mathrm{x} \in \mathrm{X} / \varphi(\mathrm{x})=\mathrm{c}\}$, para todo $\mathrm{c} \in \mathbb{R}$. Tal condição garante a transversalidade entre o hiperplano $\mathcal{W}\left(\bar{t}_{1}, \mathrm{x}_{0}, \mathcal{U}\right)$ e os hiperplanos $[\varphi: c]=\{x \in X / \varphi(x)=c\}$. Sem esta condição, como acabamos de mostrar, para algum $c_{0} \in \mathbb{R}$, teríamos $\left.\mathcal{L}(\overline{\mathrm{t}})\right|_{u_{\Gamma}}=$ constante, caso em que não teríamos um problema de otimização, e $\mathcal{A}(\varphi, \mathrm{c}) \cap \mathcal{U}_{\Gamma}=\varnothing$, para todo $\mathrm{c} \neq \mathrm{c}_{0}$. Como nos interessam apenas os estados que são atingidos pelo sistema, optamos pela primeira formulação. A figura a seguir representa os conjuntos definidos anteriormente

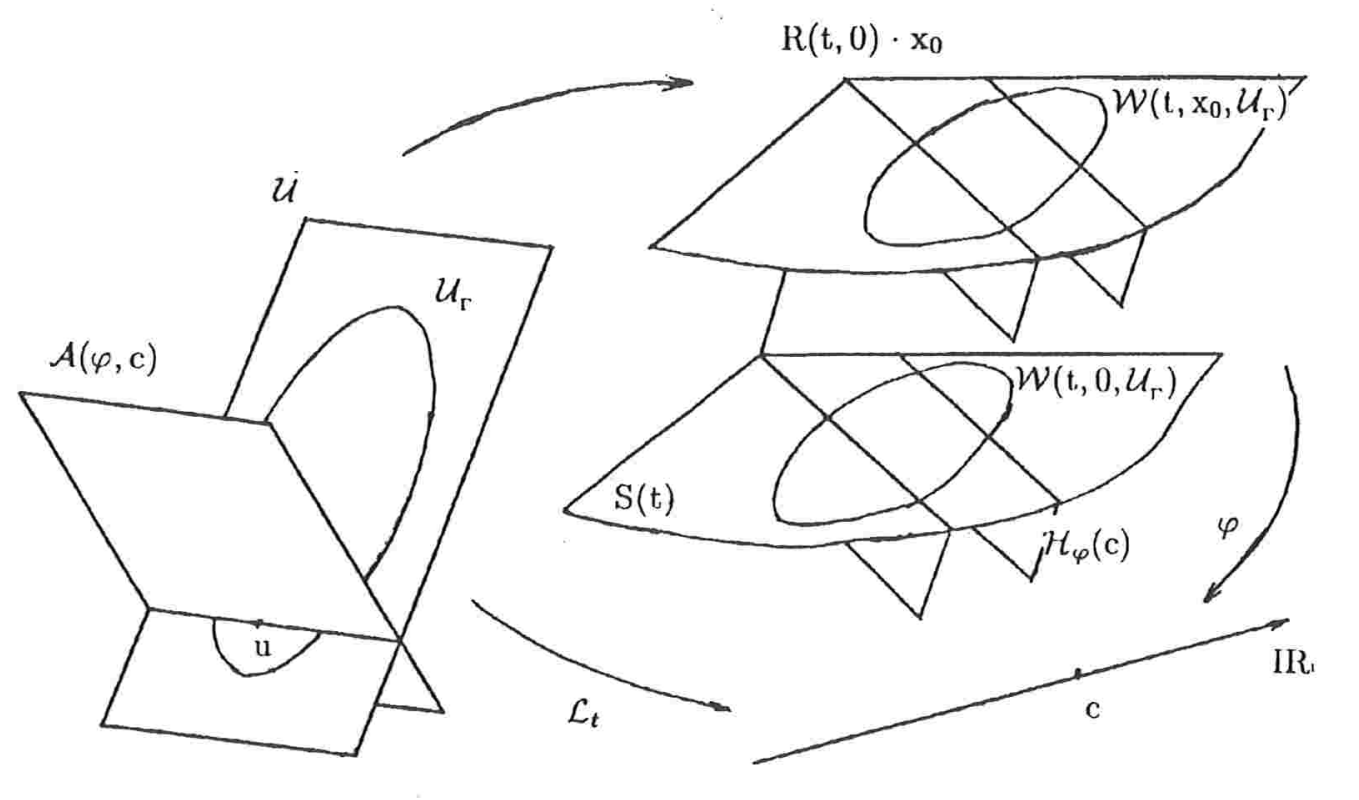

figura 3.1

3.2.3 Observação Fixado $\varphi \in \mathrm{S}(\overline{\mathrm{t}})^{\prime}$ 
a. Para cada $\mathrm{c} \in \mathbb{R}$, os conjuntos $\mathcal{A}(\varphi, \mathrm{c})$ são fechados e convexos.

b. Se $c_{1} \neq c_{2}$, então $\mathcal{A}\left(\varphi, c_{1}\right) \cap \mathcal{A}\left(\varphi, c_{2}\right)=\varnothing$.

Nosso objetivo é mostrar que os conjuntos $\mathcal{A}(\varphi, \mathrm{c})$ interceptam $\mathcal{U}_{\Gamma}$ e que existe um $c_{\max }$ tal que o controle $\mathrm{u} \in \mathcal{A}\left(\varphi, c_{\max }\right) \cap \mathcal{U}_{\Gamma}$ é um controle ótimo para o problema $(\mathrm{K})+\left(\mathrm{F}_{\alpha}\right)+(\Gamma)$. Vamos começar mostrando algumas propriedades dos conjuntos $\mathcal{A}(\varphi, \mathrm{c})$.

3.2.4 Proposição Seja $\mathrm{u}_{0}(\mathrm{t})=0$, para todo $\mathrm{t} \in[0, \mathrm{~T}]$. Então $\mathrm{u}_{0} \in \mathcal{A}(\varphi, \mathrm{c})$ se, e somente se, $\mathrm{c}=[\varphi \circ \mathrm{R}(\mathrm{t}, 0)] \cdot \mathrm{x}_{0} . \quad$ Em particular, $\mathrm{u}_{0} \in \mathcal{A}(\varphi, 0)$ se, e somente se. $\mathrm{x}_{0} \in \operatorname{Ker}[\varphi \circ \mathrm{R}(\mathrm{t}, 0)]$. Além disso, se $\mathrm{x}_{0} \in \operatorname{Ker} \mathrm{R}(\mathrm{t}, 0)$, então $\mathrm{u}_{0} \in \mathcal{A}(\varphi, 0)$.

Prova : Se $\mathrm{u}_{0} \equiv 0$, temos que, para $0 \leq \mathrm{t} \leq \mathrm{T}$, como $\mathcal{F} \mathrm{u}=0, \mathrm{x}_{\mathrm{u}_{0}}(\mathrm{t})=\mathrm{R}(\mathrm{t}, 0) \cdot \mathrm{x}_{0}$, e portanto,

$$
\mathcal{L}(\mathrm{t}) \mathrm{u}_{0}=<\varphi, \mathrm{R}(\mathrm{t}, 0) \cdot \mathrm{x}_{0}>=<\varphi \circ \mathrm{R}(\mathrm{t}, 0), \mathrm{x}_{0}>
$$

donde seguem os resultados.

Já vimos no lema 2.2 .2 que, para $\gamma \in \Gamma$. os controles constantes $\mathbf{u}_{\gamma} \equiv \gamma$ pertencem a $\mathcal{U}_{\Gamma}$. Uma questão que surge naturalmente é : para que $\mathrm{c} \in \mathbb{R}$ o hiperplano $\mathcal{A}(\varphi, \mathrm{c})$ contém $\mathbf{u}_{\gamma}$, e portanto, se os conjuntos $\mathcal{A}(\varphi, \mathrm{c})$ interceptam $\mathcal{U}_{\Gamma}$.

3.2.5 Proposição Se $\gamma \in \Gamma$, então existe $c \in \mathbb{R}$ tal que $\mathcal{A}(\varphi, c) \cap \mathcal{U}_{\Gamma} \neq \emptyset$. Na verdade $c=[\varphi \circ R(\overline{\mathrm{t}}, 0)] \cdot\left(\mathrm{x}_{0}+\gamma\right), \overline{\mathrm{t}}$ fixo.

Prova : Se $\mathrm{u}_{\gamma}(\mathrm{t})=\gamma$, para todo $\mathrm{t} \in[0, \mathrm{~T}]$. então a resposta correspondente $\mathrm{x}_{\mathrm{u}_{\gamma}}$, para 
cada $\mathrm{t} \in[0, \mathrm{~T}]$, é dada por

$$
\mathrm{x}_{\mathrm{u}_{\gamma}}(\mathrm{t})=\mathrm{R}(\mathrm{t}, 0) \cdot\left(\mathrm{x}_{0}+\gamma\right)
$$

(ver exemplo 1.3.2). Assim,

$$
\left.\mathcal{L}(\mathrm{t}) \mathrm{u}_{\gamma}=<\varphi, \mathrm{x}_{\mathrm{u}_{\gamma}}(\overline{\mathrm{t}})\right\rangle=[\varphi \circ \mathrm{R}(\mathrm{t}, 0)] \cdot\left(\mathrm{x}_{0}+\gamma\right) .
$$

Lembrando que $u_{\gamma} \in \mathcal{U}_{\Gamma}$ se, e somente se, $\gamma \in \Gamma$, temos o resultado.

3.2.6 Observação Se $\gamma \in \Gamma$ e $u_{\gamma}(\bar{t})=\gamma$, para todo $\mathrm{t} \in[0, \mathrm{~T}]$, então segue da proposição anterior que $\mathrm{u}_{\gamma} \in \mathcal{A}(\varphi, \mathrm{c}) \cap \mathcal{U}_{\Gamma}$, para $\mathrm{c}=[\varphi \circ \mathrm{R}(\overline{\mathrm{t}}, 0)] \cdot\left(\mathrm{x}_{0}+\gamma\right)$, o que acontece se, e somente se,

$$
\mathrm{R}(\overline{\mathrm{t}}, 0) \cdot\left(\mathrm{x}_{0}+\gamma\right) \in \mathcal{H}_{\varphi}(\mathrm{c})
$$

3.2.7 Observação Para todo $y \in \mathrm{S}_{\overline{\mathrm{t}}}$, em particular para $\mathrm{y} \in \mathcal{W}\left(\overline{\mathrm{t}}, 0, \mathcal{U}_{\Gamma}\right)$, se $\varphi \in \mathrm{S}_{\overline{\mathrm{t}}}^{\prime}$, então existe $\mathrm{c}_{\mathrm{y}} \in \mathbb{R}$ tal que $\mathrm{y} \in \mathcal{H}_{\varphi}\left(\mathrm{c}_{\mathrm{y}}\right)$, já que $\mathrm{S}_{\overline{\mathrm{t}}}=\operatorname{Ker} \varphi \oplus[\mathrm{y}]$. Além disso,

$$
\mathcal{H}_{\varphi}\left(\mathrm{c}_{\mathrm{y}}\right)=\left\{\mathrm{z} \in \mathrm{S}_{\overline{\mathrm{t}}} / \mathrm{z}=\mathrm{y}+\mathrm{v}, \mathrm{v} \in \operatorname{Ker} \varphi\right\}
$$

e, fixado $y_{0} \in \mathcal{W}\left(\overline{\mathrm{t}}, 0 . \mathcal{U}_{\mathrm{r}}\right), y_{0} \neq 0$, temos que $\varphi(\mathrm{z})=\lambda \varphi\left(\mathrm{y}_{0}\right)$, para todo $\mathrm{z} \in \mathcal{W}\left(\overline{\mathrm{t}}, 0, \mathcal{U}_{\mathrm{r}}\right)$. Claro que a cada $\mathrm{y} \in \mathcal{H}_{\varphi}(\mathrm{c}) \cap \mathcal{W}\left(\overline{\mathrm{t}}, 0, \mathcal{U}_{\Gamma}\right)$ corresponde um controle $\mathrm{u} \in \mathcal{A}\left(\varphi, c_{\mathrm{y}}\right) \cap \mathcal{U}_{\Gamma}$.

Vamos estudar agora a existência e localização de controles extremais. A proposição 2.3.21 garante que se $u \in \partial_{\text {rel }} \mathcal{U}_{\Gamma}$, então u é um controle $\Gamma$-extremal. Como $\mathrm{x}_{\mathrm{u}}(\overline{\mathrm{t}}) \in \mathcal{H}_{\varphi}(\mathrm{c})$, para algum $c \in \mathbb{R}$. temos que $u \in \mathcal{A}($ ヶ.$c) \cap \mathcal{U}_{\Gamma}$. 
3.2.8 Proposição Seja $\mathrm{c} \in \mathbb{R}$ tal que $\mathcal{A}(\varphi, \mathrm{c}) \cap \mathcal{U}_{\Gamma} \neq \emptyset$ Então existe um controle $\Gamma$-extremal em $\mathcal{A}(\varphi, c) \cap \mathcal{U}_{\Gamma}$, ist.o é, existe $u \in \mathcal{A}(\varphi, \mathrm{c})$ tal que

$$
\mathrm{y}=\mathrm{x}_{\mathrm{u}}(\overline{\mathrm{t}}) \in \mathcal{H}_{\varphi}(\mathrm{c}) \cap \partial_{\mathrm{S}(\overline{\mathrm{t}})} \mathcal{W}\left(\overline{\mathrm{t}}, 0, \mathcal{U}_{\Gamma}\right)
$$

Prova : Como $\mathcal{U}_{\mathrm{r}}$ é fechado, podemos considerar um controle u $\in \mathcal{A}(\varphi, \mathrm{c}) \cap \partial_{\text {rel }} \mathcal{U}_{\mathrm{r}} \neq \varnothing$. A proposição 2.3.21 acarreta que $\mathrm{y}=\mathrm{x}_{\mathrm{u}}\left(\overline{\mathrm{t}}_{\mathrm{t}}\right) \in \partial_{\mathrm{S}(\overline{\mathrm{t}})} \mathcal{W}\left(\overline{\mathrm{t}}_{1}, 0, \mathcal{U}_{\Gamma}\right)$, isto é, u é um controle $\Gamma$-extremal. Além disso, pela observação 3.2 .1 temos que y $\in \mathcal{H}_{\varphi}(\mathrm{c})$.
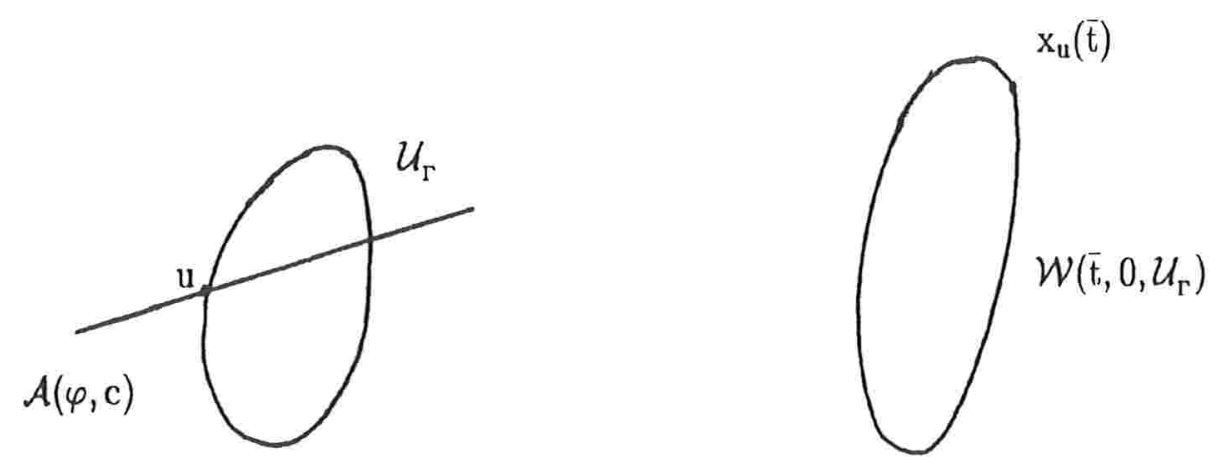

figura 3.2

\subsection{Um Princípio do Máximo}

Um Princípio do Máximo é o tipo de resultado sempre perseguido em Teoria do Controle Ótimo. O objetivo é exibir uma condição necessária e suficiente que um controle deve satisfazer para ser um controle ótimo. O Princípio do Máximo mais conhecido é devido a Pontryagin(v. [11])

Embora não seja do tipo Pontryagin, o próximo tcorema cumpre este objetivo no caso 
do sistema $(\mathrm{K})+\left(\mathrm{F}_{\alpha}\right)+(\Gamma)$. Na verdade, o próximo teorema caracteriza os controles $\Gamma$-extremais do problema $(\mathrm{K})+\left(\mathrm{F}_{\alpha}\right)+(\Gamma)$, em cada instante $\overline{\mathrm{t}} \in[0, T]$. Lembramos que fixado o núcleo $\mathrm{K} \in \mathrm{G}_{0}^{\sigma} \cdot \mathrm{SV}^{\mathrm{u}}$, denotamos por $\mathrm{R} \in \mathrm{G}_{\mathrm{I}}^{\sigma} \cdot \mathrm{SV}^{\mathrm{u}}$ o resolvente ( único ) de $\mathrm{K}$, e

$$
\mathrm{R}\left(\overline{\mathrm{t}}_{-}, \mathrm{s}\right)=\lim _{\sigma \uparrow \overline{\mathrm{t}}} \mathrm{R}(\sigma, \mathrm{s})
$$

Antes de enunciar o próximo teorema vamos detalhar, na forma de exemplo, uma implicação do teorema 1.5.2. Tal exemplo será essencial para a demonstração do Princípio do Máximo.

3.3.1 Exemplo Seja $\bar{x} \in X$ fixo e consideremos para um instante $\bar{t} \in[0, T]$, a seguinte restrição linear, tipo condição inicial,

$$
\mathrm{x}(\overline{\mathrm{t}})=\overline{\mathrm{x}},
$$

que na forma $\left(F_{\alpha}\right)$, para $\left.x \in G^{-}([0, T], X)\right)$, fica

$$
\int_{0}^{\mathrm{T}} \cdot \mathrm{d} \alpha_{\overline{\mathrm{t}}}(\mathrm{s}) \cdot \mathrm{x}(\mathrm{s})=\overline{\mathrm{x}}
$$

sendo $\alpha_{\overline{\mathrm{t}}}(\mathrm{s}) \cdot \mathrm{z}=\left(\chi_{[\overline{\mathrm{t}}, \mathrm{T}]} \cdot \mathrm{z}\right)(\mathrm{t})$, para $\mathrm{s} \in[0, \mathrm{~T}]$ e $\mathrm{z} \in \mathrm{X}$. Se $\mathrm{x} \in \mathrm{G}([0, \mathrm{~T}], \mathrm{X})$, a condição fica

$$
\mathrm{x}\left(\overline{\mathrm{t}}_{-}\right)=\overline{\mathrm{x}}
$$

Então 
(a) A equação adjunta do sistema $(\mathrm{K})+\left(\mathrm{F}_{\alpha_{\overline{\mathrm{t}}}}\right)$ é dada por

$$
\mathrm{y}(\mathrm{s})+\int_{\mathrm{s}}^{\mathrm{T}} \cdot \mathrm{K}(\mathrm{t}, \mathrm{s})^{*} \cdot \mathrm{d} \mathrm{y}(\mathrm{t})-\int_{0}^{\mathrm{T}} \cdot \mathrm{K}(\mathrm{t}, 0)^{*} \cdot \mathrm{d} \mathrm{y}(\mathrm{t})+\left(\chi_{[\overline{\mathrm{t}}, \mathbf{T}]} \cdot \varphi\right)(\mathrm{s})=0,0 \leq \mathrm{s} \leq \mathrm{T}
$$

sendo $\left(\chi_{[\bar{t}, \mathbf{T}]} \cdot \varphi\right)(\mathrm{s}) \cdot \mathrm{x}=\left(\chi_{[\mathbf{t}, \mathbf{T}]}<\varphi, \mathrm{y}>\right)(\mathrm{s})$, para $\mathrm{y} \in \mathrm{X}$ e $\varphi \in \mathrm{X}^{\prime}$

Basta notar que

$$
\begin{gathered}
<\alpha_{\overline{\mathrm{t}}}(\mathrm{s})^{*} \varphi, \mathrm{y}>:=<\varphi, \alpha_{\overline{\mathrm{t}}}(\mathrm{s}) \cdot \mathrm{y}>=\left\{\begin{array}{ll}
<\varphi, 0>, & \text { se } 0 \leq \mathrm{s}<\overline{\mathrm{t}}, \\
<\varphi, \mathrm{y}>, & \text { se } \overline{\mathrm{t}} \leq \mathrm{s} \leq \mathrm{T} .
\end{array}=\right. \\
=\left\{\begin{array}{cc}
0, & \text { se } 0 \leq \mathrm{s}<\overline{\mathrm{t}}, \\
<\varphi, \mathrm{y}>, & \text { se } \overline{\mathrm{t}} \leq \mathrm{s} \leq \mathrm{T}
\end{array}=\left(\chi_{\overline{[}, \mathrm{T}]}<\varphi, \mathrm{y}>\right)(\mathrm{s}) .\right.
\end{gathered}
$$

(b) Pelo exemplo 1.4.3, o funcional $\mathrm{J}(0)$ é dado por

$$
\mathrm{J}(0)=\int_{0}^{\mathrm{T}} \cdot \mathrm{d} \alpha_{\overline{\mathrm{t}}}(\tau) \cdot \mathrm{R}(\tau, 0)=\mathrm{R}\left(\overline{\mathrm{t}}_{-}, 0\right)
$$

(c) $\mathrm{J}(0)^{*}{ }_{\hat{f}}=0$ se, e somente se, $\left\langle\mathrm{R}\left(\overline{\mathrm{t}}_{-}, 0\right) \hat{\varphi}, \mathrm{x}\right\rangle=0$, para todo $\mathrm{x} \in \mathrm{X}$, isto é, $\left.<\varphi, \mathrm{R}\left(\overline{\mathrm{t}}_{-}, 0\right) \cdot \mathrm{x}\right\rangle=0$, para todo $\mathrm{x} \in \mathrm{X}$, o que só ocorre quando $\operatorname{Im}\left(\mathrm{R}\left(\overline{\mathrm{t}}_{-}\right), 0\right) \subset \operatorname{Ker} \varphi$.

Desse modo, se $\varphi \in \mathrm{X}^{\prime}$ e $\mathrm{R}\left(\overline{\mathrm{t}}_{-}, 0\right) \subset \operatorname{Ker} \varphi$, e pelo teorema 1.5.2 segue que $\left(\mathrm{K}_{\alpha}^{*}\right)_{0}$ tem uma solução $\mathrm{y}_{\overline{\mathrm{t}}} \in \mathrm{BV}_{0}^{0, \mathrm{~T}}\left([0, \mathrm{~T}], \mathrm{X}^{\prime}\right) \mathrm{e}$, para $0 \leq \mathrm{s} \leq \mathrm{T}$,

$$
\mathrm{y}_{\overline{\mathrm{t}}}(\mathrm{s})=\left[\mathrm{R}\left(\overline{\mathrm{t}}_{-} \cdot \mathrm{s}\right)-\mathrm{R}\left(\overline{\mathrm{t}}_{-}, 0\right)\right]^{*} \varphi
$$

pois 


$$
\begin{gathered}
\int_{\mathrm{s}}^{\mathrm{T}} \cdot \mathrm{d} \alpha_{\overline{\mathrm{t}}}(\tau) \cdot \mathrm{R}(\tau, \mathrm{s})=\mathrm{R}\left(\overline{\mathrm{t}}_{-}, \mathrm{s}\right) \text { e } \\
\mathrm{y}_{\overline{\mathrm{t}}}=\left\{\int_{\mathrm{s}}^{\mathrm{T}} \cdot \mathrm{d} \alpha_{\overline{\mathrm{t}}}(\tau) \cdot \mathrm{R}(\tau, \mathrm{s})-\int_{0}^{\mathrm{T}} \cdot \mathrm{d} \alpha_{\overline{\mathrm{t}}}(\tau) \cdot \mathrm{R}(\tau, 0)\right\}^{*} \varphi .
\end{gathered}
$$

Consideremos agora o núcleo $\mathrm{K}$ nas condições do teorema 1.5 .2 e tal que

$$
R(\bar{t}, 0) X \text { é um subespaço vetorial fechado de X. }
$$

Então o sistema $(\mathrm{K})+\left(\mathrm{F}_{\boldsymbol{\alpha}}\right)$, para $\alpha=\alpha_{\overline{\mathrm{t}}}$, tem a propriedade da dualidade, isto é, vale a parte (a) do teorema 1.5.2. Portanto, uma condição necessária e suficiente para que (K) tenha uma solução $\mathrm{x}_{\mathrm{u}} \in \mathrm{G}([0, \mathrm{~T}], \mathrm{X})$ satisfazendo $\mathrm{x}_{\mathrm{u}}\left(\overline{\mathrm{t}}_{-}\right)=\overline{\mathrm{x}}$ é que

$$
\int_{0}^{\mathrm{T}} \cdot\left\langle\mathrm{d}\left[\varphi \circ \mathrm{R}\left(\overline{\mathrm{t}}_{-}, \tau\right)\right], \mathrm{u}(\tau)\right\rangle=\langle\varphi,-\overline{\mathrm{x}}\rangle
$$

Realmente, da expressão da solução $y_{\bar{t}}$, segue que,

$$
\begin{gathered}
\int_{0}^{\mathrm{T}} \cdot\left\langle\mathrm{d} y_{\overline{\mathrm{t}}}(\tau), \mathrm{u}(\tau)>=\int_{0}^{\mathrm{T}} \cdot\left\langle\mathrm{d}\left[\mathrm{R}\left(\overline{\mathrm{t}}_{-}, \tau\right)-\mathrm{R}\left(\overline{\mathrm{t}}_{-}, 0\right)\right]^{*} \hat{\psi}, \mathrm{u}(\tau)>:=\right.\right. \\
\int_{0}^{\mathrm{T}} \cdot<\varphi, \mathrm{d}\left[\mathrm{R}\left(\overline{\mathrm{t}}_{-}, \tau\right)-\mathrm{R}\left(\overline{\mathrm{t}}_{-}, 0\right)\right] \cdot \mathrm{u}(\tau)>=\int_{0}^{\mathrm{T}} \cdot\left\langle\varphi, \mathrm{d} \mathrm{R}\left(\overline{\mathrm{t}}_{-}, \tau\right) \cdot \mathrm{u}(\tau)>-\right. \\
\quad+\int_{0}^{\mathrm{T}} \cdot<\varphi, \mathrm{d} \mathrm{R}\left(\overline{\mathrm{t}}_{-}, 0\right) \cdot \mathrm{u}(\tau)>=<\varphi, \int_{0}^{\mathrm{T}} \cdot \mathrm{d} \mathrm{R}\left(\overline{\mathrm{t}}_{-}, \bar{\tau}\right) \cdot \mathrm{u}(\tau)>-
\end{gathered}
$$




$$
+<\varphi, \int_{0}^{\mathrm{T}} \cdot \mathrm{d} \mathrm{R}\left(\overline{\mathrm{t}}_{-}, 0\right) \cdot \mathrm{u}(\tau)>=\int_{0}^{\mathrm{T}} \cdot\left\langle\varphi, \mathrm{dR}\left(\overline{\mathrm{t}}_{-}, \mathrm{t}\right) \cdot \mathrm{u}(\tau)>\right.
$$

pois, como $\mathrm{R}\left(\overline{\mathrm{t}}_{-}, 0\right)=$ constante,

$$
\int_{0}^{\mathrm{T}} \cdot \mathrm{d} \mathrm{R}\left(\overline{\mathrm{t}}_{-}, 0\right) \cdot \mathrm{u}(\tau)=0
$$

Além disso, pela Observação 1.1.17,

$$
\int_{0}^{\mathrm{T}} \cdot\left\langle\varphi, \mathrm{dR}\left(\overline{\mathrm{t}}_{-}, \tau\right) \cdot \mathrm{u}(\tau)\right\rangle=\int_{0}^{\mathrm{T}} \cdot\left\langle\mathrm{d}\left[\varphi \circ \mathrm{R}\left(\overline{\mathrm{t}}_{-}, \tau\right)\right], \mathrm{u}(\tau)\right\rangle
$$

Finalmente, se $(u, z) \in G([0, T], X) \times X$ é tal que $(K)+\left(F_{\alpha}\right)$ tem uma solução regrada $x \in G([0, T], X)$, então $(\star)$ fica

$$
\int_{0}^{\mathrm{T}} \cdot\left\langle\mathrm{d}\left[\varphi \circ \mathrm{R}\left(\overline{\mathrm{t}}_{-}, \tau\right)\right], \mathrm{u}(\tau)>=<\varphi,-\overline{\mathrm{x}}>\right.
$$

3.3.2 Um Princípio do Máximo Seja $\varphi \in \mathrm{BV}\left([0, \mathrm{~T}] . \mathrm{S}(\overline{\mathrm{t}})^{\prime}\right)$ e suponhamos que $\mathrm{R}\left(\overline{\mathrm{t}}_{-}, 0\right) \mathrm{X}$ seja um subespaço vetorial fechado de $\operatorname{Ker} \varphi(\overline{\mathrm{t}})$. Uma condição necessária e suficiente para que um controle $\overline{\mathrm{u}} \in \mathcal{U}_{\Gamma}^{-}$leve o estado inicial $\mathrm{x}_{0}=0$ a um estado

$$
\overline{\mathrm{x}} \in \mathcal{H}_{\varphi(\mathrm{t})}\left(\mathrm{c}_{\max }\right) \cap \partial_{\mathrm{S}(\overline{\mathrm{t}})} \mathcal{W}\left(\overline{\mathrm{t}}, 0, \mathcal{U}_{\Gamma}^{-}\right)
$$

sendo $c_{\max }=\max \left\{c \in R / \mathcal{H}(\mathrm{c}) \cap \mathcal{W}\left(\overline{\mathrm{t}}, 0, \mathcal{U}_{\Gamma}\right) \neq \emptyset\right.$ é que $\overline{\mathrm{u}}$ maximize a integral, sobre $\mathcal{U}_{\Gamma}^{-}$,

$$
\int_{0}^{\bar{t}} \cdot<\mathrm{d}\left[\varphi(\bar{t}) \circ \mathrm{R}\left(\overline{\mathrm{t}}_{-}, \mathrm{s}\right)\right], \mathrm{u}(\mathrm{s})>
$$


isto é.

$$
\int_{0}^{\overline{\mathrm{t}}} \cdot\left\langle\mathrm{d}\left[\varphi(\overline{\mathrm{t}}) \circ \mathrm{R}\left(\overline{\mathrm{t}}_{-}, \mathrm{s}\right)\right], \overline{\mathrm{u}}(\mathrm{s})\right\rangle=\max _{\mathrm{u} \in \mathcal{U}_{\Gamma}} \int_{0}^{\overline{\mathrm{t}}} \cdot\left\langle\mathrm{d}\left[\varphi(\overline{\mathrm{t}}) \circ \mathrm{R}\left(\overline{\mathrm{t}}_{-}, \mathrm{s}\right)\right], \mathrm{u}(\mathrm{s})\right\rangle
$$

Prova : Fixado $\varphi(\overline{\mathrm{t}}) \in \mathrm{S}(\overline{\mathrm{t}})^{\prime}$, como

$$
\begin{aligned}
<\mathrm{J}(0)^{*} \varphi(\overline{\mathrm{t}}), \mathrm{x}>:=<\varphi(\overline{\mathrm{t}}), \mathrm{J}(0) \cdot \mathrm{x}>=<\varphi(\overline{\mathrm{t}}), \int_{0}^{\mathrm{T}} \cdot \mathrm{d} \alpha_{\overline{\mathrm{t}}}(\tau) \circ \mathrm{R}(\tau, 0) \cdot \mathrm{x}>= \\
=<\varphi(\overline{\mathrm{t}}), \mathrm{R}\left(\overline{\mathrm{t}}_{-}, 0\right) \cdot \mathrm{x}>=0,
\end{aligned}
$$

sendo $\alpha_{\overline{\mathrm{t}}}$ definida como no exemplo 1.4 .3 , consideremos

$$
\mathrm{y}_{\varphi(\overline{\mathrm{t}})}(\mathrm{s})=\left\{\int_{\mathrm{s}}^{\overline{\mathrm{t}}} \cdot \mathrm{d} \alpha_{\overline{\mathrm{t}}}(\sigma) \cdot \mathrm{R}(\sigma, \mathrm{s})-\int_{0}^{\overline{\mathrm{t}}} \cdot \mathrm{d} \alpha_{\overline{\mathrm{t}}}(\sigma) \cdot \mathrm{R}(\sigma, 0)\right\}^{*} \varphi(\overline{\mathrm{t}})
$$

a solução da equação adjunta do sistema $(K)+\left(F_{\alpha_{\bar{t}}}\right)+(\Gamma)$, isto é, para o caso em que a condição $\left(\mathrm{F}_{\alpha}\right)$ é dada por

$$
\mathrm{x}_{\mathbf{u}}\left(\overline{\mathrm{t}}_{-}\right)=\overline{\mathrm{x}}
$$

Então, como já vimos no exemplo anterior, o teorema 1.5.2 garante que um controle $\mathrm{u} \in \mathcal{U}_{\Gamma}$ leva $\mathrm{x}_{0}=0 \in \mathrm{X}$ até o estado $\mathrm{y} \in \mathcal{W}\left(\overline{\mathrm{t}}, 0, \mathcal{U}_{\Gamma}\right)$ se, e somente se,

$$
\int_{0}^{\overline{\mathrm{t}}} \cdot<\mathrm{d}\left[\varphi(\overline{\mathrm{t}}) \circ \mathrm{R}\left(\overline{\mathrm{t}}_{-}, \mathrm{s}\right), \mathrm{u}(\mathrm{s})>=\langle\varphi(\overline{\mathrm{t}}),-\mathrm{y}\rangle\right.
$$

Em particular, $\overline{\mathrm{u}}$ leva $\mathrm{x}_{0}=0 \in \mathrm{X}$ até o estado $\overline{\mathrm{x}} \in \mathcal{W}\left(\overline{\mathrm{t}} .0 . \mathcal{U}_{\mathrm{r}}\right)$ se. e somente se. 


$$
\int_{0}^{\overline{\mathrm{t}}}<<\mathrm{d}\left[\varphi(\overline{\mathrm{t}}) \circ \mathrm{R}\left(\overline{\mathrm{t}}_{-}, \mathrm{s}\right)\right], \overline{\mathrm{u}}(\mathrm{s})>=<\varphi(\overline{\mathrm{t}}),-\overline{\mathrm{x}}>
$$

Se $\overline{\mathrm{x}} \in \mathcal{H}_{\varphi(\overline{\mathrm{t}})}\left(\mathrm{c}_{\max }\right) \cap \partial_{\mathrm{S}(\mathfrak{t})} \mathcal{W}\left(\overline{\mathrm{t}}, 0, \mathcal{U}_{\mathrm{r}}\right)$, como $\mathcal{W}\left(\overline{\mathrm{t}}^{\mathrm{t}}, 0, \mathcal{U}_{\Gamma}\right) \subset \mathrm{S}(\overline{\mathrm{t}})$ é um subconjunto convexo e com interior não-vazio, pelo Teorema de Mazur ( Hahn-Banach geométrico ) segue que $\bar{x}$ suporta $\mathcal{W}\left(\overline{\mathrm{t}}, 0, \mathcal{U}_{\Gamma}\right)$ e $\mathcal{H}_{\varphi(\bar{\imath})}\left(\mathrm{c}_{\max }\right)$ é o hiperplano suporte, isto é,

$$
\left.<\varphi(\overline{\mathrm{t}}),-\overline{\mathrm{x}})>=\max _{\mathrm{y} \in \mathcal{W}\left(\left(, 0,0, U_{\Gamma}\right)\right.}<\varphi\left(\overline{\mathrm{t}}_{\mathrm{r}}\right),-\mathrm{y}\right\rangle .
$$

Portanto,

$$
\int_{0}^{\overline{\mathrm{t}}} \cdot\left\langle\mathrm { d } \left[\varphi(\overline{\mathrm{t}}) \circ \mathrm{R}\left(\overline{\mathrm{t}}_{-}, \mathrm{s}\right), \overline{\mathrm{u}}(\mathrm{s})>=\max _{\mathrm{u} \in \mathcal{U}_{\Gamma}} \int_{0}^{\overline{\mathrm{t}}} \cdot<\mathrm{d}\left[\varphi(\overline{\mathrm{t}}) \circ \mathrm{R} \cdot\left(\overline{\mathrm{t}}_{-}, \mathrm{s}\right), \mathrm{u}(\mathrm{s})>.\right.\right.\right.
$$

Por outro lado, se ù maximiza a integral em (3.10) então

$$
\left.\left\langle\varphi(\overline{\mathrm{t}}), \mathrm{x}_{\mathrm{u}}\left(\overline{\mathrm{t}}_{\mathrm{t}}\right)\right\rangle=\max _{\mathrm{u} \in \mathcal{U}_{\Gamma}}\left\langle\varphi(\overline{\mathrm{t}}), \mathrm{x}_{\mathrm{u}}(\overline{\mathrm{t}})\right\rangle=\max _{y \in \mathcal{W}\left(\mathrm{t}, 0, U_{\Gamma}\right)}<\varphi(\overline{\mathrm{t}}), \mathrm{y}\right\rangle .
$$

e portanto, $\mathrm{x}_{\overline{\mathrm{u}}}(\overline{\mathrm{t}}) \in \mathcal{H}_{\varphi(\overline{\mathrm{t}})}\left(\mathrm{c}_{\max }\right) \cap \partial_{\mathrm{S}(\overline{\mathrm{t}})} \mathcal{W}\left(\overline{\mathrm{t}}, 0, \mathcal{U}_{\Gamma}\right)$

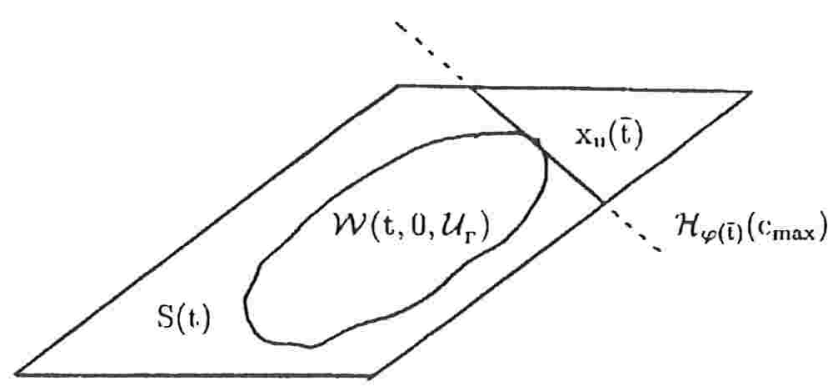

figura 3.3 
3.3.3 Observação Quando $X$ for de dimensão finita a condição sobre $R\left(\bar{t}_{-}, 0\right) X$ é sempre satisfeita.

3.3.4 Observação Se $\mathrm{x}_{\overline{\mathrm{u}}}(\overline{\mathrm{t}}) \in \mathcal{H}_{\varphi(\overline{\mathrm{t}})}\left(\mathrm{c}_{\max }\right)$, então $\overline{\mathrm{u}}$ é um controle ótimo para o funcional $\mathcal{L}_{\overline{\mathrm{t}}}$.

3.3.5 Observação $O$ controle $\overline{\mathrm{u}}$ que maximiza a integral depende do funcional $\varphi(\overline{\mathrm{t}})$ fixado, e portanto podemos denotar $\overline{\mathrm{u}}$ por $\mathrm{u}_{\varphi(\overline{\mathrm{t}})}$.

Vamos mostrar agora, suportados pelo Princípio do Máximo enunciado anteriormente, um algoritmo que permite determinar o controle ótimo. Começamos mostrando um resultado que dá informação sobre a posição do estado alvo $\overline{\mathrm{x}}=\mathrm{x}(\overline{\mathrm{t}})$ em relação ao conjunto de estados atingíveis $\mathcal{W}\left(\overline{\mathrm{t}}, \mathrm{x}_{0}, \mathcal{U}_{\Gamma}\right)$. Na verdade, o teorema a seguir caracteriza os controles $\Gamma$-extremais de $\mathcal{U}_{\Gamma}$. O teorema transfere para o nosso contexto o resultado conseguido por Wyderka em [17] para espaços de dimensão finita.

3.3.6 Teorema(Wyderka) Suponhamos que $\mathrm{R}\left(\overline{\mathrm{t}}_{-} .0\right) \mathrm{X}$ seja um subespaço fechado. Uma condição necessária e suficiente para que um controle $\overline{\mathrm{u}} \in \mathcal{U}_{\Gamma}^{-}$leve o sistema $(\mathrm{K})+\left(\mathrm{F}_{\alpha}\right)$ do estado inicial $\mathrm{x}_{0}$ a um estado

$$
\overline{\mathrm{x}} \in \partial_{\mathrm{S}(\overline{\mathrm{t}})} \mathcal{W}\left(\overline{\mathrm{t}}, \mathrm{x}_{0}, \mathcal{U}_{\mathrm{r}}\right)
$$

é que

$$
\max _{\|\varphi(\overline{\mathrm{t}})\|=1} \frac{<\varphi(\overline{\mathrm{t}}),-\overline{\mathrm{x}}>}{\int_{0}^{\overline{\mathrm{t}}}<\mathrm{d}\left[\varphi(\overline{\mathrm{t}}) \circ \mathrm{R}\left(\overline{\mathrm{t}}_{-}, \mathrm{s}\right)\right], \overline{\mathrm{u}}(\mathrm{s})>}=1 .
$$


Prova : Suponhamos que $\varphi_{\mathbf{0}}(\overline{\mathrm{t}}) \in \mathrm{S}(\overline{\mathrm{t}})^{\prime}$ seja o funcional para o qual

$$
\frac{\left\langle\varphi_{0}(\overline{\mathrm{t}}),-\overline{\mathrm{x}}>\right.}{\int_{0}^{\overline{\mathrm{t}}}<\mathrm{d}\left[\varphi_{0}(\overline{\mathrm{t}})(\overline{\mathrm{t}}) \circ \mathrm{R}\left(\overline{\mathrm{t}}_{-}, \mathrm{s}\right)\right], \overline{\mathrm{u}}(\mathrm{s})>}=\max _{\|\varphi(\overline{\mathrm{t}})(\mathrm{t})\|=1} \frac{<\varphi(\overline{\mathrm{t}}),-\overline{\mathrm{x}}>}{\int_{0}^{\mathrm{t}}<\mathrm{d}\left[\varphi(\overline{\mathrm{t}}) \circ \mathrm{R}\left(\overline{\mathrm{t}}_{-}, \mathrm{s}\right)\right], \overline{\mathrm{u}}(\mathrm{s})>}=1
$$

sendo $\overline{\mathrm{u}}$ o controle correspondente a $\varphi_{0}(\overline{\mathrm{t}})$ no sentido do Princípio do Máximo. Então

$$
\left\langle\varphi_{0}(\overline{\mathrm{t}}),-\overline{\mathrm{x}}\right\rangle=\int_{0}^{\overline{\mathrm{t}}} \cdot\left\langle\mathrm{d}\left[\varphi_{0}(\overline{\mathrm{t}}) \circ \mathrm{R}\left(\overline{\mathrm{t}}_{-}, \mathrm{s}\right)\right], \overline{\mathrm{u}}(\mathrm{s})\right\rangle
$$

Como $<\varphi_{0}(\overline{\mathrm{t}}) \cdot-\overline{\mathrm{x}}>$ é constante, segue que $\overline{\mathrm{u}}$ maximiza a integral, em $\mathcal{U}_{\Gamma}$, isto é,

$$
\int_{0}^{\overline{\mathrm{t}}} \cdot\left\langle\mathrm{d}\left[\varphi_{0}(\overline{\mathrm{t}}) \circ \mathrm{R}\left(\overline{\mathrm{t}}_{-}, \mathrm{s}\right)\right], \overline{\mathrm{u}}(\mathrm{s})>=\max _{\mathrm{u} \in \mathcal{U}_{\Gamma}} \int_{0}^{\overline{\mathrm{t}}} \cdot\left\langle\mathrm{d}\left[\varphi_{0}(\overline{\mathrm{t}}) \circ \mathrm{R}\left(\overline{\mathrm{t}}_{-}, \mathrm{s}\right)\right], \mathrm{u}(\mathrm{s})>.\right.\right.
$$

Assim, pelo Princípio do Máximo, temos que $\overline{\mathrm{u}}$ leva o estado inicial $\mathrm{x}_{0}$ ao estado $\overline{\mathrm{x}}=\mathrm{x}_{\overline{\mathrm{u}}}(\overline{\mathrm{t}}), \mathrm{com}$

$$
\overline{\mathrm{x}}=\mathrm{x}_{\overline{\mathrm{u}}}(\overline{\mathrm{t}}) \in \partial_{\mathrm{S}(\overline{\mathrm{t}})} \mathcal{W}\left(\overline{\mathrm{t}} . \mathrm{x}_{0}, \mathcal{U}_{\mathrm{r}}\right)
$$

Suponhamos agora que $\overline{\mathrm{x}} \in \partial_{\mathrm{S}(\overline{\mathrm{t}})} \mathcal{W}\left(\overline{\mathrm{t}} . \mathrm{x}_{0}, \mathcal{U}_{\mathrm{r}}\right)$. Se $\varphi \in \mathrm{BV}\left([0, \mathrm{~T}], \mathrm{S}(\overline{\mathrm{t}})^{\prime}\right)$ é tal que $\|\varphi(\overline{\mathrm{t}})\|=1$, e $\overline{\mathrm{x}} \in \mathcal{H}_{\varphi(\overline{\mathrm{t}})}(\mathrm{c}) \cap \partial_{\mathrm{S}(\overline{\mathrm{t}})} \mathcal{W}\left(\overline{\mathrm{t}}, \mathrm{x}_{0}, \mathcal{U}_{\Gamma}\right)$, então o Princípio do Máximo garante que

$$
\langle\varphi(\overline{\mathrm{t}}) \cdot-\overline{\mathrm{x}}\rangle=\max _{\mathrm{u} \in \mathcal{U}_{\Gamma}} \int_{0}^{\overline{\mathrm{t}}} \cdot\left\langle\mathrm{d}\left[\varphi(\overline{\mathrm{t}}) \circ \mathrm{R}\left(\overline{\mathrm{t}}_{-} \cdot \mathrm{s}\right)\right], \mathrm{u}(\mathrm{s})\right\rangle=\int_{0}^{\overline{\mathrm{t}}} \cdot\left\langle\mathrm{d}\left[\varphi(\overline{\mathrm{t}}) \circ \mathrm{R}\left(\overline{\mathrm{t}}_{-} \cdot \mathrm{s}\right)\right], \mathrm{u}_{\varphi}(\mathrm{s})\right\rangle
$$

ou equivalentemente, 


$$
\frac{\langle\varphi(\overline{\mathrm{t}}),-\overline{\mathrm{x}}>}{\int_{\mathbf{0}}^{\overline{\mathrm{t}}}<\mathrm{d}\left[\varphi(\overline{\mathrm{t}}) \circ \mathrm{R}\left(\overline{\mathrm{t}}_{-}, \mathrm{s}\right)\right], \mathrm{u}_{\varphi(\overline{\mathrm{t}})}(\mathrm{s})>}=1
$$

sendo $\mathrm{u}_{\varphi(\overline{\mathrm{t}})}(\mathrm{s})$ o controle correspondente, via Princípio do Máximo, ao funcional $\varphi(\bar{t})$.

Resta então mostrar que

$$
\frac{<\varphi(\overline{\mathrm{t}}),-\overline{\mathrm{x}}>}{\int_{0}^{\overline{\mathrm{t}}}<\mathrm{d}\left[\varphi(\overline{\mathrm{t}}) \circ \mathrm{R}\left(\overline{\mathrm{t}}_{-}, \mathrm{s}\right)\right], \mathrm{u}_{\varphi(\overline{\mathrm{t}})}(\mathrm{s})>}=\max _{\|\psi(\overline{\mathrm{t}})\|=1} \frac{<\psi(\overline{\mathrm{t}}),-\overline{\mathrm{x}}>}{\int_{0}^{\overline{\mathrm{t}}}<\mathrm{d}\left[\psi(\overline{\mathrm{t}}) \circ \mathrm{R}\left(\overline{\mathrm{t}}_{-}, \mathrm{s}\right)\right], \mathrm{u}_{\psi(\overline{\mathrm{t}})}(\mathrm{s})>}
$$

Consideremos então um funcional $\psi \in \mathrm{BV}\left([0, \mathrm{~T}], \mathrm{S}(\overline{\mathrm{t}})^{\prime}\right)$ de modo que $\psi(\overline{\mathrm{t}}) \neq \varphi(\overline{\mathrm{t}})$, $\|\psi(\overline{\mathrm{t}})\|=1 \mathrm{e} \mathrm{com}$

$$
\overline{\mathrm{x}} \in \mathcal{H}_{\psi(\overline{\mathrm{t}})}(\mathrm{c})
$$

Se $\mathcal{H}_{\psi(\overline{\mathrm{t}})}(\mathrm{c})$ suporta $\mathcal{W}\left(\overline{\mathrm{t}}, 0, \mathcal{U}_{\mathbf{\Gamma}}\right)$ em $\overline{\mathrm{x}}$, então a igualdade (3.17) também vale para $\psi(\bar{t})$, isto é,

$$
\frac{\langle\psi(\overline{\mathrm{t}}) \cdot-\overline{\mathrm{x}}>}{\int_{0}^{\overline{\mathrm{t}}}<\mathrm{d}\left[\psi(\overline{\mathrm{t}}) \circ \mathrm{R}\left(\overline{\mathrm{t}}_{-}, \mathrm{s}\right)\right], \mathrm{u}_{\psi}(\overline{\mathrm{t}})(\mathrm{s})>}=1
$$

Se $\mathcal{H}_{\psi(\overline{\mathrm{t}})}(\mathrm{c})$ não suporta $\mathcal{W}\left(\overline{\mathrm{t}}, 0, \mathcal{U}_{\Gamma}\right)$, então

$$
\mathcal{H}_{u(\overline{\mathrm{t}})}(\mathrm{c}) \cap \operatorname{int}_{\mathrm{S}(\overline{\mathrm{t}})} \mathcal{W}\left(\overline{\mathrm{t}} .0 . \mathcal{U}_{\mathrm{r}}\right) \neq \varnothing
$$

Logo existem $c_{\max }(\dot{\psi}(\overline{\mathrm{t}}))=\overline{\mathrm{c}}$ e um estado $\mathrm{x}_{1}$ tais que $\mathcal{H}_{\bar{t}^{t}(\overline{\mathrm{t}})}(\overline{\mathrm{c}})$ suporta $\mathcal{W}\left(\overline{\mathrm{t}}, 0, \mathcal{U}_{\boldsymbol{\Gamma}}\right)$ em $\mathrm{x}_{1}$, isto é, 


$$
\mathrm{x}_{1} \in \mathcal{H}_{\psi(\overline{\mathrm{t}})}(\overline{\mathrm{c}}) \cap \partial_{\mathrm{S}(\overline{\mathrm{t}})} \mathcal{W}\left(\overline{\mathrm{t}}, 0, \mathcal{U}_{\Gamma}\right)
$$

Pelo Princípio do Máximo, o controle $\mathrm{u}_{\psi}\left(\overline{\mathrm{t}}_{\mathrm{t}}\right)$ que leva o sistema $(\mathrm{K})+\left(\mathrm{F}_{\alpha}\right)$ de $\mathrm{x}_{0}$ ao estado $\mathrm{x}_{1}$ é tal que,

$$
\begin{aligned}
\overline{\mathrm{c}}=\left\langle\psi(\overline{\mathrm{t}}),-\mathrm{x}_{1}\right\rangle & =\int_{0}^{\overline{\mathrm{t}}}<\mathrm{d}\left[\psi\left(\overline{\mathrm{t}}_{\mathrm{t}}\right) \circ \mathrm{R}\left(\overline{\mathrm{t}}_{-}, \mathrm{s}\right)\right], \mathrm{u}_{\psi}(\mathrm{s})>=\max _{\mathrm{u} \in \mathcal{U}_{\Gamma}} \int_{0}^{\overline{\mathrm{t}}} \cdot\left\langle\mathrm{d}\left[\psi(\overline{\mathrm{t}}) \circ \mathrm{R}\left(\overline{\mathrm{t}}_{-}, \mathrm{s}\right)\right], \mathrm{u}(\mathrm{s})\right\rangle> \\
& >\int_{0}^{\overline{\mathrm{t}}} \cdot\left\langle\mathrm{d}\left[\psi(\overline{\mathrm{t}}) \circ \mathrm{R}\left(\overline{\mathrm{t}}_{-}, \mathrm{s}\right)\right], \mathrm{u}_{\varphi}(\mathrm{s})\right\rangle=\langle\psi(\overline{\mathrm{t}}),-\overline{\mathrm{x}}\rangle=\mathrm{c} .
\end{aligned}
$$

Portanto,

$$
\frac{\langle\psi(\overline{\mathrm{t}}),-\overline{\mathrm{x}}>}{\left\langle\psi(\overline{\mathrm{t}}),-\mathrm{x}_{1}>\right.}<1, \text { e então, }
$$

figura 3.4 
Suponhamos agora que $\overline{\mathrm{x}} \notin \mathcal{W}\left(\overline{\mathrm{t}}_{\mathrm{t}} \mathrm{x}_{0}, \mathcal{U}_{\Gamma}\right)$. Como int $\mathrm{S}(\overline{\mathrm{t}}) \mathcal{W}\left(\overline{\mathrm{t}}_{\mathrm{t}}, \mathrm{x}_{0}, \mathcal{U}_{\Gamma}\right) \neq \varnothing$ e o subconjunto $\mathcal{W}\left(\overline{\mathrm{t}}, \mathrm{x}_{0}, \mathcal{U}_{\Gamma}\right)$ é convexo, o Teorema de Hahn-Banach garante que existe um funcional $\varphi^{0}(\overline{\mathrm{t}}) \in \mathrm{S}(\overline{\mathrm{t}})^{\prime} \operatorname{com}\left\|\varphi^{0}(\overline{\mathrm{t}})\right\|=1$, não identicamente nulo, tal que

$$
<\varphi^{0}\left(\overline{\mathrm{t}}_{\mathrm{t}}\right), \overline{\mathrm{x}}>\leq \inf _{\mathrm{y} \in \mathcal{W}\left(\mathrm{i}, 0, u_{\Gamma}\right)}<\varphi^{0}\left(\overline{\mathrm{t}}_{\mathrm{t}}\right), \mathrm{y}>
$$

ou, equivalentemente, $\mathcal{W}\left(\overline{\mathrm{t}}_{\mathrm{t}}, \mathrm{x}_{0}, \mathcal{U}_{\Gamma}\right)$ e $\{\overline{\mathrm{x}}\}$ podem ser separados por um hiperplano $\mathcal{H}_{\varphi^{0}(\overline{\mathrm{t}})}(\mathrm{c})$ de $\mathrm{S}(\overline{\mathrm{t}})$, para algum $\mathrm{c} \in \mathbb{R}$, de modo que $\overline{\mathrm{x}} \notin \mathcal{H}_{\varphi^{0}(\overline{\mathrm{t}})}(\mathrm{c})$.

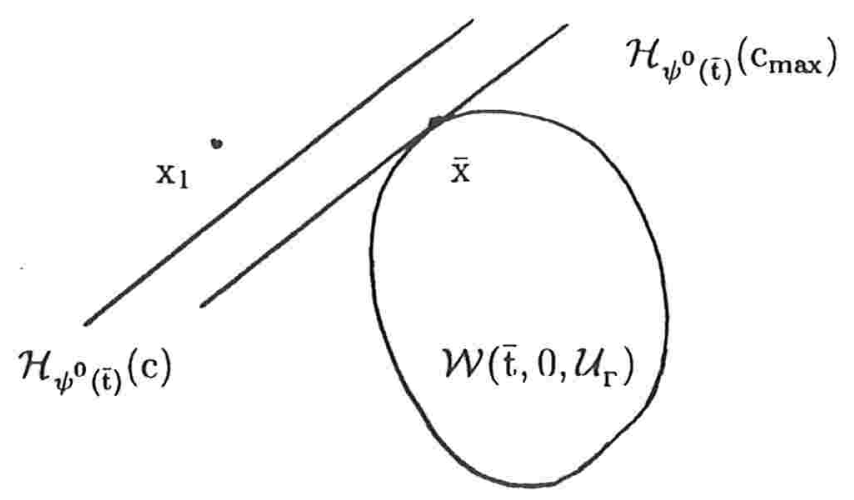

figura 3.5

Fixado $\varphi^{0}(\overline{\mathrm{t}})$, seja $c_{\max } \in \mathbb{R}$ tal que $\mathcal{H}_{\varphi^{0}(\overline{\mathrm{t}})}\left(c_{\max }\right)$ suporta $\mathcal{W}\left(\overline{\mathrm{t}}, 0, \mathcal{U}_{\Gamma}\right)$ em $\mathrm{x}^{0}\left(\overline{\mathrm{t}}_{1}\right)$, onde $\mathrm{x}^{0}$ é a resposta correspondente, via fórmula $(\rho)$, ao controle $\mathrm{u}^{0}$. Assim,

$$
<\varphi^{0}(\overline{\mathrm{t}}), \overline{\mathrm{x}}>=<\varphi^{0}(\overline{\mathrm{t}}), \mathrm{x}^{0}(\overline{\mathrm{t}})>=\int_{0}^{\overline{\mathrm{t}}}<\mathrm{d}\left[\varphi^{0}(\overline{\mathrm{t}}) \circ \mathrm{R}\left(\overline{\mathrm{t}}_{-}, \mathrm{s}\right)\right], \mathrm{u}^{0}(\mathrm{~s})>
$$

e

$$
\mathrm{x}^{0}(\overline{\mathrm{t}}) \in \mathcal{H}_{\varphi^{0}(\overline{\mathrm{t}})}\left(\mathrm{c}_{\max }\right) \cap \mathcal{W}\left(\overline{\mathrm{t}}, 0, \mathcal{U}_{\Gamma}\right)
$$


Portanto,

$$
\frac{<\varphi^{0}(\overline{\mathrm{t}}), \overline{\mathrm{x}}>}{\int_{0}^{\bar{t}}<\mathrm{d}\left[\varphi^{0}(\overline{\mathrm{t}}) \circ \mathrm{R}\left(\overline{\mathrm{t}}_{-}, \mathrm{s}\right)\right], \mathrm{u}^{0}(\mathrm{~s})>}>1
$$

e consequentemente,

$$
\max _{\|\varphi(\overline{\mathrm{t}})\|=1} \frac{<\varphi(\overline{\mathrm{t}}), \overline{\mathrm{x}}>}{\int_{0}^{\mathrm{t}}<\mathrm{d}\left[\varphi(\overline{\mathrm{t}}) \circ \mathrm{R}\left(\overline{\mathrm{t}}_{-}, \mathrm{s}\right)\right], \mathrm{u}^{0}(\mathrm{~s})>}>1 .
$$

Reciprocamente, se

$$
\max _{\|\varphi(\mathrm{t})\|=1} \frac{<\varphi(\overline{\mathrm{t}}), \overline{\mathrm{x}}>}{\int_{0}^{\overline{\mathrm{t}}}<\mathrm{d}\left[\varphi(\overline{\mathrm{t}}) \circ \mathrm{R}\left(\overline{\mathrm{t}}_{-}, \mathrm{s}\right)\right], \mathrm{u}^{0}(\mathrm{~s})>}>1,
$$

então existe um funcional não identicamente nulo $\varphi^{0}(\overline{\mathrm{t}}) \in \mathrm{S}(\overline{\mathrm{t}})^{\prime}$, com $\left\|\varphi^{0}(\overline{\mathrm{t}})\right\|=1$, tal que

$$
\frac{<\varphi^{0}(\overline{\mathrm{t}}), \overline{\mathrm{x}}>}{\int_{0}^{\bar{t}}<\mathrm{d}\left[\varphi^{0}(\overline{\mathrm{t}}) \circ \mathrm{R}\left(\overline{\mathrm{t}}_{-}, \mathrm{s}\right)\right], \mathrm{u}^{0}(\mathrm{~s})>}>1,
$$

ou seja,

$$
\left\langle\varphi^{0}(\overline{\mathrm{t}}), \overline{\mathrm{x}}>>\int_{0}^{\overline{\mathrm{t}}} \cdot\left\langle\mathrm{d}\left[\varphi^{0}(\overline{\mathrm{t}}) \circ \mathrm{R}\left(\overline{\mathrm{t}}_{-}, \mathrm{s}\right)\right], \mathrm{u}^{0}(\mathrm{~s})>=\mathrm{k}\right.\right.
$$

Como $\mathrm{u}^{0}$ maximiza a integral, pelo Princípio do Máximo devemos, no instante $\bar{t}$, atingir um estado

$$
\mathrm{x}^{0}(\overline{\mathrm{t}}) \in \mathcal{H}_{\varphi}^{\mathrm{o}(\overline{\mathrm{t}})}(\mathrm{c}) \cap \mathcal{W}\left(\overline{\mathrm{t}}, 0, \mathcal{U}_{\mathrm{r}}\right)
$$


tal que $\left\langle\varphi^{0}(\overline{\mathrm{t}}), \mathrm{x}^{0}(\overline{\mathrm{t}})\right\rangle=\mathrm{k}$. Logo $\left\langle\varphi^{0}(\overline{\mathrm{t}}), \overline{\mathrm{x}}\right\rangle\left\langle\left\langle\varphi^{0}(\overline{\mathrm{t}}), \mathrm{x}^{0}(\overline{\mathrm{t}})\right\rangle\right.$, donde segue que $\overline{\mathrm{x}}$ pertence ao hiperplano $\left.\mathcal{H}_{\varphi^{0}(\overline{\mathrm{t}})}(\mathrm{c}), \operatorname{com} \mathrm{c}=<\varphi^{\mathrm{o}}(\overline{\mathrm{t}}), \overline{\mathrm{x}}\right\rangle$, que não intercepta o conjunto de pontos atingíveis $\mathcal{W}\left(\overline{\mathrm{t}}, 0, \mathcal{U}_{\Gamma}\right)$, já que $\mathrm{c}>\mathrm{k}$. Portanto

$$
\overline{\mathrm{x}} \notin \mathcal{W}\left(\overline{\mathrm{t}}, 0, \mathcal{U}_{\mathrm{r}}\right)
$$

Consideremos agora o seguinte

3.3.7 Lema Se $\left\{Y_{\lambda}\right\}_{\lambda \in\{a, b j}$ é uma família paramétrica de conjuntos compactos do espaço de Banach $\mathrm{X}$, que depende continuamente de $\lambda$, e se

$$
\overline{\mathrm{x}} \notin \mathrm{Y}_{\mathrm{a}} \text { e } \overline{\mathrm{x}} \in \mathrm{Y}_{\mathrm{b}}
$$

então existe um parâmetro $\mathrm{c} \in] \mathrm{a}, \mathrm{b}[$ tal que

$$
\overline{\mathrm{x}} \in \partial \mathrm{Y}_{\mathrm{c}}
$$

Além disso, se a dependência $\lambda \longmapsto \mathrm{Y}_{\lambda}$ for estritamente monôtona no sentido da inclusão, isto é, se $\xi<\eta$ implica $\mathrm{Y}_{\xi} \subset \mathrm{Y}_{\eta}$, então c é unicamente determinado.

3.3.8 Observação $\mathrm{O}$ teorema anterior deixa claro que, quando se quer garantir otimalidade de funcionais, os estados $\bar{x} \in$ int $_{\text {rel }} \mathcal{W}\left(t, x_{0}, \mathcal{U}_{\Gamma}\right)$ são indesejáveis. Em [17], Wyderka propõe um método de bissecção que tem como suporte o lema anterior e, no nosso caso, da proposição 2.3.11. Intuitivamente, o método garante que podemos calibrar a potência dos controles $\mathcal{U}_{\Gamma}$, isto é, diminuir $\Gamma$, de modo a garantir que um dado estado $\overline{\mathrm{x}}$ pertença à fronteira de $\mathcal{W}\left(\mathrm{t}, \mathrm{x}_{0}, \mathcal{U}_{\mathrm{r}}\right)$. 
Lembramos que na observação 2.3.13 destacamos a continuidade (em relação à métrica de Hausdorff) e a monotonicidade (no sentido da inclusão) da aplicação

$$
\lambda \in[0,1] \rightarrow \mathcal{W}\left(\mathrm{t}, \mathrm{x}_{0}, \lambda \mathcal{U}_{\mathrm{r}}\right)
$$

quando $\mathrm{x}_{0} \in \operatorname{Ker} \mathrm{R}(\mathrm{t}, 0)$ e para $\mathrm{t} \in[0, \mathrm{~T}]$ fixado.

Sejam $\bar{x} \in X, \bar{x} \neq 0$ e $\overline{\mathrm{t}}<\infty$ o primeiro instante em que $\overline{\mathrm{x}} \in \mathcal{W}\left(\overline{\mathrm{t}}, \mathrm{x}_{0}, \mathcal{U}_{\mathbf{r}}\right)$. Vamos considerar no lema 3.3.7, para $\lambda \in[0,1]$,

$$
\mathrm{Y}_{\lambda}=\mathcal{W}\left(\overline{\mathrm{t}}, \mathrm{x}_{0}, \lambda \mathcal{U}_{\Gamma}\right)
$$

e suponhamos que $\mathrm{x}_{0} \in \operatorname{Ker} \mathrm{R}(\overline{\mathrm{t}}, 0)$. Então

$$
\begin{gathered}
\mathrm{Y}_{0}=\{0\}, \overline{\mathrm{x}} \notin \mathrm{Y}_{0}, \\
\mathrm{Y}_{1}=\mathcal{W}\left(\mathrm{T}, \mathrm{x}_{0}, \mathcal{U}_{\Gamma}\right) \text { e } \overline{\mathrm{x}} \in \mathrm{Y}_{1}
\end{gathered}
$$

Então, pelo lema 3.3.7, existe um único $\bar{\lambda} \in \mid 0,1[$ tal que

$$
\overline{\mathrm{x}} \in \partial_{\text {rel }} \mathcal{W}\left(\overline{\mathrm{t}}, \mathrm{x}_{0}, \bar{\lambda} \mathcal{U}_{\mathrm{r}}\right)
$$

Podemos então. determinado o instante $\overline{\mathrm{t}} \in[0, \mathrm{~T}]$ tal que $\overline{\mathrm{x}} \in \mathcal{W}\left(\overline{\mathrm{t}}, \mathrm{x}_{0}, \mathcal{U}_{\Gamma}\right)$, encontrar 
o controle ótimo para o problema $(\mathcal{P})_{0, \bar{\imath}, x_{0}}$ usando o seguinte algoritmo :

(i). Se $\overline{\mathrm{x}} \in \partial_{\text {rel }} \mathcal{W}\left(\overline{\mathrm{t}}, \mathrm{x}_{0}, \mathcal{U}_{\Gamma}\right)$, então o controle ótimo é caracterizado pelo Princípio do Máximo.

(ii). Caso contrário, isto é, se $\overline{\mathrm{x}} \in$ int $_{\mathrm{rel}} \mathcal{W}\left(\overline{\mathrm{t}}, \mathrm{x}_{0}, \mathcal{U}_{\Gamma}\right)$, procuramos $\left.\lambda_{1}, \lambda_{2} \in\right] 0,1[$ tais que

$$
\overline{\mathrm{x}} \notin \mathcal{W}\left(\overline{\mathrm{t}}, \mathrm{x}_{0}, \lambda_{1} \mathcal{U}_{\mathrm{r}}\right) \text { e } \overline{\mathrm{x}} \in \mathcal{W}\left(\overline{\mathrm{t}}, \mathrm{x}_{0}, \lambda_{2} \mathcal{U}_{\mathrm{r}}\right)
$$

(iii). Agora fazemos $\lambda=\frac{1}{2}\left[\lambda_{1}+\lambda_{2}\right]$ e usamos o teorema 3.3 .6 para verificar

a. Se $\overline{\mathrm{x}} \notin \mathcal{W}\left(\overline{\mathrm{t}}, \mathrm{x}_{0}, \bar{\lambda} \mathcal{U}_{\Gamma}\right)$. Neste caso, consideramos

$$
\lambda_{1}=\lambda \text { e } \lambda_{2}=\lambda_{2}
$$

e voltamos a (iii).

b. Se $\overline{\mathrm{x}} \in \operatorname{int}_{\mathrm{rel}} \mathcal{W}\left(\overline{\mathrm{t}}, \mathrm{x}_{0}, \lambda \mathcal{U}_{\mathrm{r}}\right)$. Neste caso, consideramos

$$
\lambda_{1}=\lambda_{1} \text { e } \lambda_{2}=\lambda
$$

e voltamos a (iii).

c. Se $\partial_{\text {rel }} \mathcal{W}\left(\overline{\mathrm{t}} \cdot \mathrm{x}_{0}, \lambda \mathcal{U}_{\mathrm{r}}\right)$. Neste caso, usamos o Princípio do Máximo para caracterizar o controle ótimo. 


\subsection{Funcional Avaliação Integral em $[0, \bar{t}]$}

Seja $\bar{t} \in[0, T]$ fixado. Vamos considerar agora o problema de controle ótimo $(\mathcal{P})_{0, \bar{t}, x_{0}}$, como no início deste capítulo, para o caso em que o funcional custo $\mathcal{L}$ é dado na forma integral

$$
\mathcal{L}(0, \overline{\mathrm{t}}) \mathrm{u}=\int_{0}^{\overline{\mathrm{t}}} \cdot<\mathrm{d} \gamma(\mathrm{s}), \mathrm{x}_{\mathrm{u}}(\mathrm{s})>
$$

sendo $y \in \mathrm{BV}_{0}\left([0, \mathrm{~T}], \mathrm{X}^{\prime}\right)$. Este tipo de funcional avalia cumulativamente o desempenho do estado durante toda a sua evolução.

Usando a fórmula $(\rho)$ da seção $1.3(\mathrm{v}$. Teor.1.3.3 ) podemos escrever uma expressão em u para o funcional $\mathcal{L}(0, \overline{\mathrm{t}})$.

3.4.1 Observação Para cada $\overline{\mathrm{t}} \in[0, \mathrm{~T}]$, o funcional $\mathcal{L}(0, \overline{\mathrm{t}})$ é dado pela fórmula

$$
\mathcal{L}(0, \overline{\mathrm{t}}) \mathrm{u}=\int_{0}^{\overline{\mathrm{t}}} \cdot<\mathrm{d} \gamma(\mathrm{s}),(\mathcal{F} \mathrm{u})(\mathrm{s})>+\mathrm{R}_{0}
$$

sendo $\mathcal{F}$ a aplicação dada na seção 2.3 e

$$
\mathrm{R}_{0}=\int_{0}^{\overline{\mathrm{t}}} \cdot\left\langle\mathrm{d} \gamma(\mathrm{s}), \mathrm{R}(\mathrm{s}, 0) \cdot \mathrm{x}_{0}\right\rangle
$$

Claro que $\mathcal{L}(0, \overline{\mathrm{t}})$ não é linear. No entanto $\mathcal{L}(0, \overline{\mathrm{t}})$ é contínua pois

$$
\|\mathcal{L}(0, \overline{\mathrm{t}}) \mathrm{u}\| \leq \mathrm{BV}[\mathrm{r}]\{1+\|\mathrm{R}(\overline{\mathrm{t}} .0)\|\}\|\mathrm{u}\|
$$


Vamos denotar por

$$
\mathcal{I}_{\Gamma}(\overline{\mathrm{t}})=\left\{\int_{\mathbf{0}}^{\overline{\mathrm{t}}} \cdot<\mathrm{d} \gamma(\mathrm{s}), \mathrm{x}_{\mathrm{u}}(\mathrm{s})>/ \mathrm{u} \in \mathcal{U}_{\Gamma}\right\} \subset \mathbb{R} .
$$

Se denotarmos por $\mathcal{F}_{\gamma}: \mathrm{G}([0, \mathrm{~T}], \mathrm{X}) \rightarrow \mathbb{R}$ a aplicação

$$
\left(\mathcal{F}_{\gamma} \mathrm{u}\right)(\mathrm{t})=\int_{\mathbf{0}}^{\mathrm{t}} \cdot\langle\mathrm{d} \gamma(\mathrm{s}),(\mathcal{F} \mathrm{u})(\mathrm{s})>
$$

então

$$
\mathcal{L}(0, \overline{\mathrm{t}}) \mathrm{u}=\left(\mathcal{F}_{\gamma} \mathrm{u}\right)(\overline{\mathrm{t}})+\mathrm{R}_{0}
$$

e

$$
\mathcal{I}_{\Gamma}(\overline{\mathrm{t}})=\left(\mathcal{F}_{\gamma} \mathcal{U}_{\Gamma}\right)(\overline{\mathrm{t}})+\mathrm{R}_{0}
$$

Como $\mathcal{U}_{\Gamma}$ é fechado e limitado, e $\mathrm{R}_{0}$ não depende de u, segue da continuidade de $\mathcal{F}_{\gamma_{\gamma}}$ que $\mathcal{I}_{\Gamma}(\overline{\mathrm{t}})$ é um subconjunto compacto da reta. Além disso, como $\mathcal{I}_{\Gamma}(\overline{\mathrm{t}})$ é convexo. se $\Gamma$ for convexo, temos que $\mathcal{I}_{\Gamma}(\overline{\mathrm{t}})$ é um intervalo.

Portanto existe

$$
\xi^{*}(\overline{\mathrm{t}})=\max _{\mathrm{u} \in \mathcal{U}_{\Gamma}} \mathcal{I}_{\Gamma}(\overline{\mathrm{t}})
$$

isto é, existe $\xi_{\times} \in \mathcal{I}_{\Gamma}(\overline{\mathrm{t}})$ tal que $\xi \leq \xi_{\times}$, para todo $\xi \in \mathcal{I}_{\Gamma}(\bar{t})$

Seja $u^{*}$ o controle tal que 


$$
\mathcal{L}(0, \overline{\mathrm{t}}) \mathrm{u}=\xi^{*}(\overline{\mathrm{t}})
$$

Então, para cada $\bar{t} \in[0, T]$ fixado, podemos considerar o sistema $(\mathrm{K})+\left(\mathrm{F}_{\gamma}\right)_{*}$,

$$
\begin{aligned}
& \mathrm{x}(\mathrm{t})-\mathrm{x}_{0}+\int_{0}^{\mathrm{t}} \cdot \mathrm{d}_{\mathrm{s}} \mathrm{K}(\mathrm{t}, \mathrm{s}) \cdot \mathrm{x}(\mathrm{s})=\mathrm{u}(\mathrm{t}), 0 \leq \mathrm{t} \leq \mathrm{T}<\infty, \\
& \mathrm{F}_{\gamma}[\mathrm{x}]=\int_{0}^{\overline{\mathrm{t}}} \cdot \mathrm{d} \gamma(\mathrm{s}) \cdot \mathrm{x}(\mathrm{s})=\xi^{*}(\overline{\mathrm{t}}),
\end{aligned}
$$

para $\mathrm{K} \in \mathrm{G}_{0}^{\sigma} \cdot \mathrm{SV}^{\mathrm{u}}([0, \mathrm{~T}] \times[0, \mathrm{~T}], \mathrm{L}(\mathrm{X})), \mathrm{x}, \mathrm{u} \in \mathrm{G}([0, \mathrm{~T}], \mathrm{X})$, com a restrição nos controles $\mathrm{u} \in \mathcal{U}_{\Gamma}$ e $\mathrm{x}_{0} \in \mathrm{X}$. Desse modo, $\mathrm{F}_{\gamma}\left[\mathrm{x}_{\mathrm{u}}\right]=\mathcal{L}(0, \overline{\mathrm{t}}) \mathrm{u}=\xi^{*}(\overline{\mathrm{t}})$ e podemos assim caracterizar o controle $\mathrm{u}^{*}$ usando a condição $(\star)$ do teorema 1.5.2.

A equação adjunta do sistema $(\mathrm{K})+\left(\mathrm{F}_{\gamma}\right)$ é dada por

$$
\mathrm{y}(\mathrm{s})+\int_{\mathrm{s}}^{\mathrm{T}} \cdot \mathrm{K}(\mathrm{t}, \mathrm{s})^{*} \cdot \mathrm{d} \mathrm{y}(\mathrm{t})-\int_{0}^{\mathrm{T}} \cdot \mathrm{K}(\mathrm{t}, 0)^{*} \cdot \mathrm{d} \mathrm{y}(\mathrm{t})+\gamma(\mathrm{s})^{*} \cdot \eta=\mathrm{h}(\mathrm{s}), 0 \leq \mathrm{s} \leq \mathrm{T},
$$

sendo h, $\mathrm{y} \in \mathrm{BV}_{0}^{0, \mathrm{~T}}\left([0, \mathrm{~T}], \mathrm{X}^{\prime}\right)$ e $\eta \in \mathbb{R}$ ( lembramos que estamos considerando $\mathrm{Y}=\mathbb{R}$ no Teorema 1.5.2, e que portanto $\mathrm{Y}^{\prime} \approx \mathbb{R}$ e os funcionais $\varphi: \mathbb{R} \rightarrow \mathbb{R}$ são dados por $\varphi(\xi)=\langle\eta, \xi>=\eta \xi)$. Além disso,

$$
<\gamma(\mathrm{s})^{*} \cdot \eta, \xi>:=<\eta, \gamma(\mathrm{s}) \cdot \xi>=\eta \gamma(\mathrm{s}) \cdot \xi=\gamma(\mathrm{s}) \cdot(\eta \xi)
$$

Neste caso, temos que o funcional $\mathrm{J}(0): \mathrm{X} \rightarrow \mathbb{R}$ é dado por 


$$
\mathrm{J}(0)=\sigma \int_{0}^{\overline{\mathrm{t}}} \cdot \mathrm{d} \gamma(\mathrm{t}) \circ \mathrm{R}(\mathrm{t}, 0) \in \mathrm{X}^{\prime}
$$

$\mathrm{e}$

$$
<\mathrm{J}(0)^{*} \cdot \eta, \xi>=<\eta, \mathrm{J}(0) \cdot \xi>=<\eta, \int_{0}^{\overline{\mathrm{t}}} \cdot \mathrm{d} \gamma(\mathrm{t}) \circ \mathrm{R}(\mathrm{t}, 0) \cdot \xi>=\eta \int_{0}^{\overline{\mathrm{t}}} \cdot \mathrm{d} \gamma(\mathrm{t}) \circ \mathrm{R}(\mathrm{t}, 0) \cdot \xi
$$

Lembremos que quando $Y=\mathbb{R}$ o teorema 1.5.2 fica

3.4.2 Teorema Suponhamos que $\mathrm{K} \in \mathrm{G}_{0}^{\sigma} \cdot \mathrm{SV}^{\mathrm{u}}\left(\Gamma_{0}, \mathrm{~L}(\mathrm{X})\right)$ tem um único resolvente $\mathrm{R}$ e que $\left.\gamma \in \mathrm{BV}_{0}\left([0, \mathrm{~T}], \mathrm{X}^{\prime}\right)\right)$. Então são equivalentes :

a. Para $(u, \xi) \in G([0, T], X) \times \mathbb{R}$ o sistema $(K)+\left(F_{\gamma}\right)$ tem uma solução $x \in G([0, T \mid, X)$ se, e somente se, para todo $\operatorname{par}(\mathrm{y}, \eta) \in \mathrm{BV}_{0}^{0, \mathrm{~T}}\left([0, \mathrm{~T}], \mathrm{X}^{\prime}\right) \times \mathbb{R}$ que satisfaz $\left(\mathrm{K}_{\gamma}^{*}\right)_{0}$ temos

$$
\int_{0}^{\mathrm{T}} \cdot<\mathrm{dy}(\mathrm{t}), \mathrm{u}(\mathrm{t})>+\eta \xi=0
$$

b. $J(0) X$ é um subespaço vetorial fechado de $\mathbb{R}$.

3.4.3 Observação Claro que, para $\mathrm{Y}=\mathbb{R}$, $\operatorname{dim} \mathrm{J}(0) \mathrm{X}<\infty$, e portanto $\mathrm{J}(0) \mathrm{X}$ é um subespaço vetorial fechado de $\mathbb{R}$. Na verdade, $J(0) X=\{0\}$ ou $J(0) X=\mathbb{R}$.

Portanto o sistema $(\mathrm{K})+\left(\mathrm{F}_{\gamma}\right)_{*}$ tem a propriedade da dualidade, isto é, uma condição necessária e suficiente para que $(K)$ tenha uma solução $x_{u} \in G([0, T], X)$ e tal que $\mathrm{x}_{\mathrm{u}}(\overline{\mathrm{t}})=\xi^{*}$ é que para todo par $(\mathrm{y}, \eta) \in \mathrm{BV}_{0}^{0, \mathrm{~T}}\left([0, \mathrm{~T}], \mathrm{X}^{\prime}\right) \times \mathbb{R}$ que satisfaz 


$$
\mathrm{y}(\mathrm{s})+\int_{\mathrm{s}}^{\mathrm{T}} \cdot \mathrm{K}(\mathrm{t}, \mathrm{s})^{*} \cdot \mathrm{d} \mathrm{y}(\mathrm{t})-\int_{0}^{\mathrm{T}} \cdot \mathrm{K}(\mathrm{t}, 0)^{*} \cdot \mathrm{d} \mathrm{y}(\mathrm{t})+\gamma(\mathrm{s})^{*} \cdot \eta=0,0 \leq \mathrm{s} \leq \mathrm{T}, \quad\left(\mathrm{K}_{\gamma}^{*}\right)_{0}
$$

tenhamos

$$
\int_{0}^{\mathrm{T}} \cdot<\mathrm{dy}(\mathrm{t}), \mathrm{u}(\mathrm{t})>=-\eta \xi^{*}
$$

Como no parágrafo 2.2 , podemos considerar a aplicação linear contínua

$$
\Psi_{\mathrm{K}, \gamma}: \mathrm{u} \in \mathrm{G}([0, \mathrm{~T}], \mathrm{X}) \longmapsto \int_{0}^{\mathrm{T}} \cdot<\mathrm{d}_{\mathrm{K}, \gamma}(\mathrm{t}), \mathrm{u}(\mathrm{t})>\in \mathbb{R} .
$$

sendo $\mathrm{y}_{\mathrm{K}, \gamma} \in \mathrm{BV}_{0}^{0, \mathrm{~T}}\left([0, \mathrm{~T}], \mathrm{X}^{\prime}\right)$, que leva à definição dos seguintes subconjuntos de $\mathrm{G}([0, \mathrm{~T}], \mathrm{X})$ :

$$
\begin{gathered}
\mathcal{V}=\mathcal{V}_{\mathrm{K}, \gamma}=\operatorname{Ker} \Psi_{\mathrm{K}, \gamma} \\
\mathcal{V}_{\lambda}=\mathcal{V}_{\mathrm{K}, \gamma, \lambda}=\left\{\mathrm{u} \in \mathrm{G}([0, \mathrm{~T}], \mathrm{X}) / \Psi_{\mathrm{K}, \gamma}(\mathrm{u})=\lambda\right\}, \lambda \in \mathbb{R} . \\
\mathcal{V}_{\Gamma}=\mathcal{V}_{\mathrm{K}, \gamma, \Gamma}=\mathcal{V}_{\mathrm{K}, \gamma} \cap \mathrm{G}([0, \mathrm{~T}], \Gamma) \\
\mathcal{V}_{\lambda, \Gamma}=\mathcal{V}_{\mathrm{K}, \gamma \lambda, \Gamma}=\mathcal{V}_{\mathrm{K}, \gamma, \lambda} \cap \mathrm{G}([0, \mathrm{~T}], \Gamma)
\end{gathered}
$$

3.4.4 Observação Valem aqui as mesmas transferências de propriedades de $\Gamma$ ao conjunto $\mathcal{V}_{\Gamma}$ discutidas no capítulo 2 . Claro que, pela observação 3.4 .3 , se $(y, \eta)$ estiverem 
nas condições do teorema 3.4 .2 , o controle $\mathrm{u}^{*} \in \mathcal{V}_{\lambda, \Gamma}$ que satisfaz a condição $(*)$ maximiza o funcional $\mathcal{L}(0, \overline{\mathrm{t}})$ em $\mathcal{V}_{\lambda, \Gamma}$. Em outras palavras, vale o

3.4.5 Teorema Suponhamos que $\mathrm{K} \in \mathrm{G}_{0}^{\sigma} \cdot \mathrm{SV}^{\mathrm{u}}\left(\Gamma_{0}, \mathrm{~L}(\mathrm{X})\right)$ tem um único resolvente $\mathrm{R}$ e que $\left.\gamma \in \mathrm{BV}_{0}\left([0, \mathrm{~T}], \mathrm{X}^{\prime}\right)\right)$ e seja $\lambda^{*}=-\eta \xi^{*}, \eta \in I R$. Uma condição necessária e suficiente para que um controle $\mathrm{u}^{*}$ maximize o funcional

$$
\mathcal{L}(0, \overline{\mathrm{t}}) \mathrm{u}=\int_{0}^{\overline{\mathrm{t}}} \cdot<\mathrm{d} \gamma(\mathrm{s}), \mathrm{x}_{\mathrm{u}}(\mathrm{s})>
$$

é que $u^{*}$ dê o valor máximo em $\mathcal{V}_{\lambda^{*}, \Gamma}$ de

$$
\mathrm{I}(0) \cdot \mathrm{u}=-\int_{0}^{\overline{\mathrm{t}}} \cdot \mathrm{d}_{\mathrm{s}}\left[\int_{\mathrm{s}}^{\overline{\mathrm{t}}} \cdot \mathrm{d} \gamma(\tau) \cdot \mathrm{R}(\tau, \mathrm{s})\right] \cdot \mathrm{u}(\mathrm{s})
$$

Prova : Pela observação 3.4 .3 temos que, para $(u, \xi) \in \mathrm{G}([0, \mathrm{~T}], \mathrm{X}) \times \mathbb{R}$, o sistema $(\mathrm{K})+\left(\mathrm{F}_{\gamma}\right)+(\Gamma)$, para

$$
\mathrm{F}_{\gamma}[\mathrm{x}]=\xi
$$

tem uma solução $x_{u} \in G([0, T] . X)$. Então pelo lema 1.5.6, para $Y=\mathbb{R}$, temos que $\xi=\mathrm{J}(0) \mathrm{x}_{0}+\xi_{\mathrm{u}}$, sendo

$$
\xi_{\mathrm{u}}=-\mathrm{J}(0) \mathrm{u}(0)-\int_{0}^{\overline{\mathrm{t}}} \cdot \mathrm{d}_{\mathbf{s}}\left[\int_{\mathrm{s}}^{\overline{\mathrm{t}}} \cdot \mathrm{d} \gamma(\tau) \cdot \mathrm{R}(\tau, \mathrm{s})\right] \cdot \mathrm{u}(\mathrm{s})=-\int_{0}^{\overline{\mathrm{t}}} \cdot \mathrm{d}_{\mathrm{s}}\left[\int_{\mathrm{s}}^{\overline{\mathrm{t}}} \cdot \mathrm{d} \gamma(\tau) \cdot \mathrm{R}(\tau, \mathrm{s})\right] \cdot \mathrm{u}(\mathrm{s})
$$

pois $\mathrm{u}(0)=0$. Assim, se $\overline{\mathrm{u}}$ maximiza $\mathrm{I}(0)$ em $\mathcal{V}_{\lambda^{*}, \Gamma}$ então

$$
\bar{\xi}=\xi_{\overline{\mathrm{u}}}=\max _{\mathrm{u} \in \mathcal{V}_{\lambda^{*}, \Gamma}} \xi_{\mathrm{u}} .
$$


Logo

$$
\mathcal{L}(0, \overline{\mathrm{t}}) \overline{\mathrm{u}}=\max _{\mathrm{u} \in \mathcal{V}_{\lambda^{*} . \Gamma}} \mathcal{L}(0, \overline{\mathrm{t}}) \mathrm{u}
$$

3.4.6 Um Princípio de Otimalidade Seja $\overline{\mathrm{u}} \in \mathcal{V}_{\lambda^{*}, \Gamma}$ um controle ótimo para o problema $(\mathcal{P})_{0, \bar{t}, \mathbf{x}_{0}}$, sendo

$$
\mathcal{L}(0, \overline{\mathrm{t}}) \mathrm{u}=\int_{0}^{\overline{\mathrm{t}}} \cdot\left\langle\mathrm{d} \gamma(\mathrm{s}), \mathrm{x}_{\mathrm{u}}(\mathrm{s})>\right.
$$

com resposta correspondente $\mathrm{x}_{\overline{\mathrm{u}}}$. Então, para qualquer $\tilde{\mathrm{t}} \in[0, \overline{\mathrm{t}}]$, o controle $\overline{\mathrm{u}}$ também é ótimo para o problema

\section{Maximizar o funcional}

$$
\mathcal{L}(\tilde{\mathrm{t}}, \overline{\mathrm{t}}) \mathrm{u}=\int_{\overline{\mathrm{t}}}^{\overline{\mathrm{t}}} \cdot\left\langle\mathrm{d} \gamma(\mathrm{s}), \mathrm{x}_{\mathrm{u}}(\mathrm{s})\right\rangle,
$$

sujeito a

dinâmica :

$$
\mathrm{x}(\mathrm{t})-\mathrm{x}_{0}+\int_{0}^{\mathrm{t}} \cdot \mathrm{d}_{\mathrm{s}} \mathrm{K}(\mathrm{t}, \mathrm{s}) \cdot \mathrm{x}(\mathrm{s})=\mathrm{u}(\mathrm{t}), 0 \leq \mathrm{t} \leq \overline{\mathrm{t}}
$$

\section{Restrição linear :}

$$
\mathrm{F}_{\alpha}[\mathrm{x}]=\int_{0}^{\overline{\mathrm{t}}} \cdot\langle\mathrm{d} \alpha(\mathrm{s}), \mathrm{x}(\mathrm{s})>=\tilde{\mathrm{c}}
$$


sendo

$$
\tilde{\mathrm{c}}=\int_{0}^{\mathrm{i}} \cdot\left\langle\mathrm{d} \alpha(\mathrm{s}), \mathrm{x}_{\tilde{\mathrm{u}}}(\mathrm{s})>\right.
$$

Prova : Suponhamos que $\mathrm{u}^{*} \in \mathcal{U}_{\Gamma}$ é um controle com $\mathrm{x}_{\mathrm{u}^{*}}$ a solução correspondente da equação $(K)_{\bar{t}}^{\bar{t}}$, isto é,

$$
\mathrm{x}_{\mathrm{u}^{*}}(\mathrm{t})-\tilde{\mathrm{x}}+\int_{\overline{\mathrm{t}}}^{\overline{\mathrm{t}}} \cdot \mathrm{d}_{\mathrm{s}} \mathrm{K}(\mathrm{t}, \mathrm{s}) \cdot \mathrm{x}_{\mathrm{u}^{*}}(\mathrm{~s})=\mathrm{u}^{*}(\mathrm{t})-\mathrm{u}^{*}(\tilde{\mathrm{t}}), \quad \tilde{\mathrm{t}} \leq \mathrm{t} \leq \overline{\mathrm{t}}
$$

sendo $\tilde{\mathrm{x}}=\mathrm{x}_{\tilde{\mathrm{u}}}(\tilde{\mathrm{t}})$, satisfazendo

$$
\mathrm{F}_{\alpha}[\mathrm{x}]=\int_{\overline{\mathbf{t}}}^{\overline{\mathrm{t}}} \cdot \mathrm{d} \alpha(\mathrm{s}) \cdot \mathrm{x}_{\mathrm{u}^{*}}(\mathrm{~s})=0
$$

e tal que

$$
\int_{\overline{\mathrm{t}}}^{\overline{\mathrm{t}}} \cdot\left\langle\mathrm{d} \gamma(\mathrm{s}), \mathrm{x}_{\mathrm{u}^{*}}(\mathrm{~s})>>\int_{\overline{\mathrm{t}}}^{\overline{\mathrm{t}}} \cdot\left\langle\mathrm{d} \gamma(\mathrm{s}), \mathrm{x}_{\overline{\mathrm{u}}}(\mathrm{s})>\right.\right.
$$

isto é, $\overline{\mathrm{u}}$ não maximiza $\mathcal{L}(\tilde{\mathrm{t}}, \overline{\mathrm{t}})$.

\section{Consideremos o controle}

$$
\tilde{\mathrm{u}}(\mathrm{t})=\left\{\begin{array}{cc}
\overline{\mathrm{u}}(\mathrm{t}), & \text { se } 0 \leq \mathrm{t} \leq \tilde{\mathrm{t}} \\
\mathrm{u}^{*}(\mathrm{t}), & \text { se } \tilde{\mathrm{t}}<\mathrm{t} \leq \overline{\mathrm{t}}
\end{array}\right.
$$

Então $\tilde{\mathrm{u}} \in \mathcal{U}_{\Gamma}, \tilde{\mathrm{u}}(0)=0$ e $\tilde{\mathbf{u}}$ produz a solução( ver figura 3.7 )

$$
\mathrm{x}_{\tilde{\mathrm{u}}}(\mathrm{t})=\left\{\begin{array}{cc}
\mathrm{x}_{\overline{\mathrm{u}}}(\mathrm{t}), & \text { se } 0 \leq \mathrm{t} \leq \tilde{\mathrm{t}} \\
\mathrm{x}_{\mathrm{u}^{*}}(\mathrm{t}), & \text { se } \tilde{\mathrm{t}}<\mathrm{t} \leq \overline{\mathrm{t}}
\end{array}\right.
$$


da equação

$$
\mathrm{x}(\mathrm{t})-\mathrm{x}_{0}+\int_{0}^{\mathrm{t}} \cdot \mathrm{d}_{\mathrm{s}} \mathrm{K}(\mathrm{t}, \mathrm{s}) \cdot \mathrm{x}_{\mathrm{u}^{*}}(\mathrm{~s})=\tilde{\mathrm{u}}(\mathrm{t}), \quad 0 \leq \mathrm{t} \leq \overline{\mathrm{t}}
$$

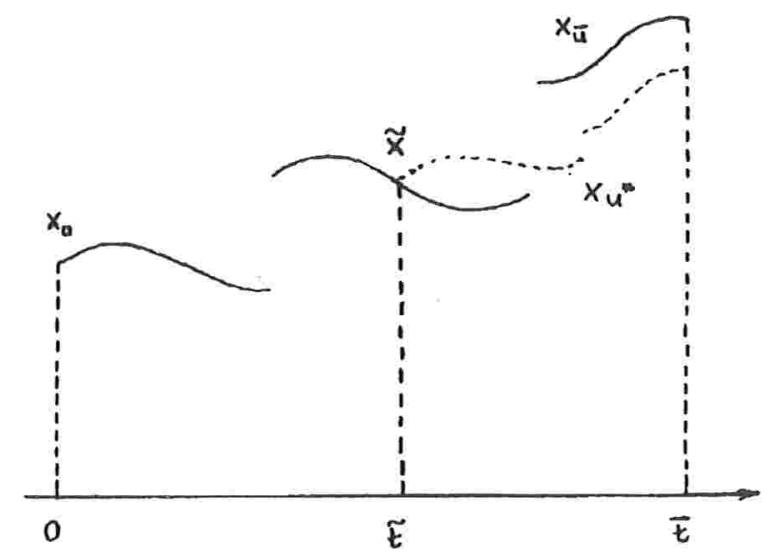

figura 3.7

De fato, para $t \in[0, \tilde{\mathrm{t}}]$ isto é claro pois $\mathrm{x}_{\tilde{\mathrm{i}}}=\mathrm{x}_{\mathrm{i}}$. Para $\mathrm{t} \in[\tilde{\mathrm{t}}, \overline{\mathrm{t}}]$, como

$$
x_{\bar{u}}(\tilde{t})-x_{0}+\int_{0}^{\bar{t}} \cdot d_{s} K(\tilde{t}, s) \cdot x_{\tilde{u}}(s)=\bar{u}(\tilde{t})
$$

temos que

$$
\begin{gathered}
x_{\tilde{u}}(t)-x_{0}+\int_{0}^{t} \cdot d_{s} K(t, s) \cdot x_{\tilde{u}}(s)=x_{u^{*}}(t)-x_{0}+\int_{0}^{\tilde{t}} \cdot d_{s} K(\tilde{t}, s) \cdot x_{\tilde{u}}(s)+\int_{\tilde{t}}^{t} \cdot d_{s} K(t, s) \cdot x_{u^{*}}(s)= \\
=x_{u^{*}}(t)+\bar{u}(\tilde{t})-\underbrace{x_{\bar{u}}(\tilde{t})}_{\tilde{x}}+\int_{\tilde{t}}^{t} \cdot d_{s} K(t, s) \cdot x_{u^{*}}(s)=u^{*}(t)-u^{*}(\tilde{t})+u^{*}(\tilde{t})=\tilde{u}(t),
\end{gathered}
$$

Além disso, segue do teorema 1.1.10 que $\mathrm{x}_{\tilde{\mathrm{n}}}$ satisfaz $\mathrm{F}_{\alpha}[\mathrm{x}]=\tilde{\mathrm{c}}$. 
De fato,

$$
\int_{0}^{\bar{t}} \cdot \mathrm{d} \alpha(\mathrm{s}) \cdot \mathrm{x}_{\overline{\mathrm{u}}}(\mathrm{s})=\int_{\mathbf{0}}^{\overline{\mathrm{t}}} \cdot \mathrm{d} \alpha(\mathrm{s}) \cdot \mathrm{x}_{\overline{\mathrm{u}}}(\mathrm{s})+\int_{\overline{\mathrm{t}}}^{\overline{\mathrm{t}}} \cdot \mathrm{d} \alpha(\mathrm{s}) \cdot \mathrm{x}_{\mathrm{u}} \cdot(\mathrm{s})
$$

Finalmente,

$$
\begin{aligned}
\mathcal{L}(0, \overline{\mathrm{t}}) \tilde{\mathrm{u}}= & \int_{0}^{\overline{\mathrm{t}}} \cdot\left\langle\mathrm{d} \gamma(\mathrm{s}), \mathrm{x}_{\tilde{\mathrm{u}}}(\mathrm{s})>=\int_{0}^{\overline{\mathrm{t}}} \cdot\left\langle\mathrm{d} \gamma(\mathrm{s}), \mathrm{x}_{\overline{\mathrm{u}}}(\mathrm{s})>+\int_{\overline{\mathrm{t}}}^{\overline{\mathrm{t}}} \cdot\left\langle\mathrm{d} \gamma(\mathrm{s}), \mathrm{x}_{\mathrm{u}^{*}} \cdot(\mathrm{s})>>\right.\right.\right. \\
& >\int_{0}^{\overline{\mathrm{t}}} \cdot\left\langle\mathrm{d} \gamma(\mathrm{s}), \mathrm{x}_{\overline{\mathrm{u}}}(\mathrm{s})>+\int_{\overline{\mathrm{t}}}^{\overline{\mathrm{t}}}<\mathrm{d} \gamma(\mathrm{s}), \mathrm{x}_{\overline{\mathrm{u}}}(\mathrm{s})>=\mathcal{L}(0, \overline{\mathrm{t}}) \overline{\mathrm{u}} .\right.
\end{aligned}
$$

Assim, $\mathcal{L}(0, \overline{\mathrm{t}}) \tilde{\mathrm{u}}>\mathcal{L}(0, \overline{\mathrm{t}}) \overline{\mathrm{u}}$, o que contraria a otimalidade de $\overline{\mathrm{u}}$.

3.4.7 Observação Dada uma divisão $d: 0=t_{0}<t_{1}<t_{2}<\ldots<t_{n}=T$ de $[0, T]$, podemos considerar no teorema anterior, $\tilde{\mathrm{t}}=\mathrm{t}_{\mathrm{j}}$, para $\mathrm{j}=0,1, \ldots, \mathrm{n}-1$, e $\overline{\mathrm{t}}=\mathrm{T}$. Então se $\overline{\mathbf{u}} \in \mathcal{V}_{\lambda^{z}, \Gamma}$ maximiza o funcional $\mathcal{L}(0, T)$, então $\overline{\mathrm{u}}_{\mathrm{j}}=\left.\overline{\mathrm{u}}\right|_{i \mathrm{i}_{\mathrm{j}}, \mathrm{T}} \in \mathcal{V}_{\overline{\mathrm{c}}_{\mathrm{j}}, \Gamma}$, maximiza o funcional $\mathcal{L}\left(\mathrm{t}_{\mathrm{j}}, \mathrm{T}\right)$, isto é,

$$
\int_{\mathrm{t}_{\mathrm{j}}}^{\mathrm{T}} \cdot\left\langle\mathrm{d} \gamma(\mathrm{s}), \mathrm{x}_{\overline{\mathrm{u}}_{\mathrm{j}}}(\mathrm{s})>=\max _{\mathrm{v} \in \mathcal{V}_{\overline{\mathrm{c}}_{\mathrm{j}}, \Gamma}} \int_{\mathrm{t}_{\mathbf{j}}}^{\mathrm{T}} \cdot\left\langle\mathrm{d} \gamma(\mathrm{s}), \mathrm{x}_{\mathrm{v}}(\mathrm{s})>\right.\right.
$$

sendo

$$
\tilde{\mathrm{c}}_{\mathrm{j}}=\int_{\mathbf{0}}^{\mathrm{t}_{\mathrm{j}}} \cdot \mathrm{d} \alpha(\mathrm{s}) \cdot \mathrm{x}_{\overline{\mathrm{u}}}(\mathrm{s})
$$

\subsection{Observações Finais}

A continuação natural deste trabalho é o estudo de Programação Dinâmica e da existência de Controles Ótimos Realimentados para o problema $(\mathcal{P})_{0, \mathrm{~T}, \mathrm{x}_{0}}$. Neste sentido, o Princípio 
de Otimalidade 3.4.6 será útil quando conjugado ao estudo da existência de controles ótimos para funcionais do tipo

$$
\mathcal{L}(\overline{\mathrm{t}}, \mathrm{T}) \mathrm{u}=<\varphi, \mathrm{x}_{\mathrm{u}}(\mathrm{T})>+\int_{\overline{\mathrm{t}}}^{\mathrm{T}} \cdot\left\langle\mathrm{d} \gamma(\mathrm{s}), \mathrm{x}_{\mathrm{u}}(\mathrm{s})\right)>
$$

definido sobre o subconjunto de $G([0, T], X)$ dos controles que produzem soluções do sistema

$$
\begin{aligned}
& x(t)-\bar{x}+\int_{\bar{t}}^{t} \cdot d_{s} K(t, s) \cdot x(s)=u(t)-u(\bar{t}), \bar{t} \leq t \leq T<\infty, \\
& F_{\alpha}[x]=\int_{0}^{T} \cdot d \alpha(s) \cdot x(s)=0,
\end{aligned}
$$

para $\bar{t} \in] 0, T]$ e $\bar{x} \in X$. Aqui as soluções $x_{u}$ de $(K)_{\bar{t}}^{T}+\left(F_{\alpha}\right)_{0}$ são dadas pela fórmula

$$
\mathrm{x}_{\mathrm{u}}(\mathrm{t})=\mathrm{u}(\mathrm{t})+\mathrm{R}(\mathrm{t}, \overline{\mathrm{t}}) \cdot\left[\mathrm{x}_{0}-\mathrm{u}(\overline{\mathrm{t}})\right]-\int_{\overline{\mathrm{t}}}^{\mathrm{t}} \cdot \mathrm{d}_{\tau} \mathrm{R}(\mathrm{t}, \tau) \cdot \mathrm{u}(\tau), \quad \overline{\mathrm{t}} \leq \mathrm{t} \leq \mathrm{T}
$$

1 e $\varphi$ e $\gamma$ são como nos parágrafos 3.2 e 3.4.

Como no parágrafo 2.3 , podemos considerar a aplicação

$$
\mathcal{G}: \mathrm{G}([0, \mathrm{~T}], \mathrm{X}) \rightarrow \mathrm{G}([0, \mathrm{~T}], \mathrm{X})
$$

definida. para $t \in[\mathrm{t}, \mathrm{T}]$, por

$$
(\mathcal{G} \mathrm{u})(\mathrm{t})=\mathrm{u}(\mathrm{t})-\mathrm{R}(\mathrm{t}, \overline{\mathrm{t}}) \cdot \mathrm{u}(\overline{\mathrm{t}})-\int_{\overline{\mathrm{t}}}^{\mathrm{t}} \cdot \mathrm{d}_{\tau} \mathrm{R}(\mathrm{t}, \overline{\mathrm{t}}) \cdot \mathrm{u}(\tau)
$$

Nosso processo de controle agora pode ser visto como 


$$
\mathrm{u} \in \mathrm{G}([0, \mathrm{~T}], \mathrm{X}) \longmapsto \mathcal{G}(\mathrm{u})(\mathrm{t})+\overline{\mathrm{X}}
$$

sendo

$$
\overline{\mathrm{X}}=\{\mathrm{R}(\mathrm{t}, \overline{\mathrm{t}}) \cdot \overline{\mathrm{x}}\}, \overline{\mathrm{x}} \in \mathrm{X} \text { fixo. }
$$

A partir da existência de um controle ótimo, para cada par $(\bar{t}, \bar{x}) \in[0, T] \times X$, podemos definir uma função de valor ótimo $\mathrm{V}:[0, \mathrm{~T}] \times \mathrm{X} \rightarrow \mathbb{R}$ definida por

$$
\mathrm{V}_{\varphi, \gamma}(\overline{\mathrm{t}}, \overline{\mathrm{x}})=\max _{\mathrm{u} \in \mathcal{A}}\left\{<\varphi, \mathrm{x}_{\mathrm{u}}(\mathrm{T})>+\int_{\overline{\mathrm{t}}}^{\mathrm{T}} \cdot\left\langle\mathrm{d} \gamma(\mathrm{s}), \mathrm{x}_{\mathrm{u}}(\mathrm{s})>\right\}\right.
$$

sendo $\mathrm{x}_{\mathrm{u}}$ a solução de $(\mathrm{K})_{\overline{\mathrm{t}}}^{\mathrm{T}}$ e $\mathcal{A} \subset \mathrm{G}([0, \mathrm{~T}], \mathrm{X})$ um conjunto conveniente de controles. Tal função deve satisfazer uma fórmula de recursão do tipo

$$
\mathrm{V}_{\varphi, \gamma}(\overline{\mathrm{t}}, \overline{\mathrm{x}})=\max _{\mathrm{u} \in \mathcal{V}_{\Gamma}}\left\{\mathrm{V}_{\varphi, \gamma}\left(\overline{\mathrm{t}}+\delta, \mathrm{x}_{\mathrm{u}}(\overline{\mathrm{t}}+\delta)\right)+\int_{\overline{\mathrm{t}}}^{\overline{\mathrm{t}}+\delta} \cdot<\mathrm{d} \gamma(\mathrm{s}), \mathrm{x}_{\mathrm{u}}(\mathrm{s})>\right\}
$$

para cada par $(\overline{\mathrm{t}} . \overline{\mathrm{x}}) \in[0, \mathrm{~T}] \times \mathrm{X}$. Em particular, para cada divisão $\mathrm{d}: 0=\mathrm{t}_{0}<\mathrm{t}_{1}<\mathrm{t}_{2}<$ $\ldots<\mathrm{t}_{\mathrm{n}}=\mathrm{T}$ de $[0, \mathrm{~T}]$,

$$
\mathrm{V}_{\varphi, \gamma}\left(\mathrm{t}_{\mathrm{j}-1}, \mathrm{x}_{\mathrm{j}-1}\right)=\max _{\mathrm{u} \in \mathcal{V}_{\Gamma}}\left\{\mathrm{V}_{\gamma, \gamma}\left(\mathrm{t}_{\mathrm{j}}, \mathrm{x}_{\mathrm{j}}\right)+\int_{\mathrm{t}_{\mathrm{j}-1}}^{\mathrm{t}_{\mathrm{j}}} \cdot<\mathrm{d} \gamma(\mathrm{s}), \mathrm{x}_{\mathrm{u}}(\mathrm{s})>\right\}
$$

Não podemos, em geral, garantir a diferenciabilidade da função de valor, pois $\mathrm{V}$, como definida, é uma função regrada da primeira variável. A diferenciabilidade de $\mathrm{V}$ exigiria a imposição de condições adicionais sobre $\mathrm{K}, \alpha$ e $\gamma$, e a consequente redefinição 
dos espaços onde estes são tomados. Desconhecemos a eficácia desta estratégia, que pode, eventualmente, nos levar de volta a um contexto que não necessite de equação tão geral como a equação $(K)$. Resumindo, a função de valor ótimo não satisfaz, em geral, uma equação diferencial parcial do tipo Hamilton-Jacobi-Bellman. No entanto, temos a expectativa de que um resultado que garanta a existência de controles ótimos realimentados possa ser demonstrado sem a hipótese de que V satisfaça uma equação deste tipo. 


\section{Bibliografia}

[1] Barbanti, L. : Controllability and Approximate Controllability for Linear Integral Volterra-Stietjes Equations, Lecture Notes on Pure and Applied Mathematic, 118, Marcel Decker, New York, 1989.

[2] Barbu, V., e Precupanu, Th. : Convexity and Optimization in Banach Spaces, Editura Academiei, Romania, 1986, 397 p.

[3] Conti, R. : Infinite Dimensional Linear Autonomous Controllability, Mathematics Report 82-127, Univ. of Minnesota, Minneapolis, 1982, 83 p.

[4] Falb, P.L. : Infinite Dimensional Control Problem I : On the Closure Of the Set of Attainable States for Linear Systems, Journal of Mathematical Analysis and Applications 9, 12-22, 1964.

[5] Hönig, C.S. : The Adjoint Equation of a Linear Volterra-Stieltjes Integral Equation with a Linear Constraint, Lecture Notes in Mathematics 957, 110-125, Springer, 1982.

[6] Hönig, C.S. : Aplicações da Topologia à Análise, Projeto Euclides - IMPA, Editora Edgar Blücher Ltda., Rio de Janeiro, 1976, 221 p.

[7] Hönig, C.S. : Equations Integrales Generaliseés et Aplications, Publications Mathematiques D' Orsay, Orsay, 1981, 50 p. 
[8] Hönig, C.S. : Semigroup and Semivariation II. Atas do $20^{\circ}$ Seminário Brasileiro de Análise, Sociedade Brasileira de Matemática, 1983.

[9] Hönig, C.S. : A Survey on Volterra-Stieltjes Integral Equations, Escola de Análise, SBM, IME-USP, São Paulo, 1977, 118 p.

[10] Hönig, C.S. : Volterra-Stieltjes Integral Equations, Math. Studies 16, North Holland Publ. Company, Amsterdam, 1975, 157 p.

[11] Lee, E. B., Markus, L. : Foundations of Optimal Control Theory, John Wiley and Sons, New York, 1976, 576 p.

[12] Leigh, J.R. : Functional Analysis and Linear Control Theory, Mathem. Science Engineering 156, Academic Press, London, 1980, 160 p.

[13] Macki, J., Straus, A. : An Introduction to Optimal Control Theory, Springer-Verlag, New York, 1982, 165 p.

[14] Narukawa, K. : Admissible Controllability of Vibrating Systems With Constraint Controls, SIAM Journal on Control and Optimization, v. 20 n. 6, November, 1982.

[15] Schechter, M. : Principles of Functional Analysis, Academic Press, New York, 1972, $383 \mathrm{p}$.

[16] Varaya, P.P. : Notes in Optimization, Van Nostrand Reinhold Company, New York, 1972,202 p.

[17] Wyderka, Z. : Linear Differential Equations with Measures as Coefficients and Control Theory, Scientific Publications of the University of Silesia, 1994, 136 p.

[18] Diekmann, O. et all : Perturbing evolutionary systems by step responses and cumulative outputs, Differential and Integral Equations, v. 8, n. 5, 1205-1244, 1995. 
Departamento de Matemática

Faculdade de Engenharia de Ilha Solteira - UNESP

Ilha Solteira - SP

Fone : (018) 762.3113 - ramal 191-130

Fax : (018) 762.2735

e-mail : lantonio@feis.unesp.br 